\title{
Toward the Understanding of Hydration Phenomena in Aqueous Electrolytes from the Interplay of Theory, Molecular Simulation, and Experiment
}

\author{
by \\ Ariel A. Chialvo, ${ }^{1^{*}}$ and Lukas Vlcek ${ }^{1,2}$ \\ ${ }^{1}$ Chemical Sciences Division, Geochemistry \& Interfacial Sciences Group \\ Oak Ridge National Laboratory, Oak Ridge, TN 37831-6110, U. S. A. \\ 2 Joint Institute for Computational Sciences \\ Oak Ridge National Laboratory, Oak Ridge, TN 37831-6173, U. S. A.
}

\begin{abstract}
This manuscript has been authored by UT-Battelle, LLC under Contract No. DE-AC05-00OR22725 with the U.S. Department of Energy. The United States Government retains and the publisher, by accepting the article for publication, acknowledges that the United States Government retains a non-exclusive, paidup, irrevocable, world-wide license to publish or reproduce the published form of this manuscript, or allow others to do so, for United States Government purposes. The Department of Energy will provide public access to these results of federally sponsored research in accordance with the DOE Public Access Plan (http://energy.gov/downloads/doe-public-access-plan).
\end{abstract}

\section{Special issue of Fluid Phase Equilibria}

Aqueous Solutions: Bulk Fluids and Interfaces

${ }^{\&}$ To whom correspondence should be addressed (chialvoaa@ornl.gov, FAX 865-574-4961)

May 5, 2015

(C) 2015. This manuscript version is made available under the Elsevier user license http://www.elsevier.com/open-access/userlicense/1.0/ 


\begin{abstract}
We study the microstructural analysis of aqueous electrolytes and present a detailed account of the fundamentals underlying the neutron scattering with isotopic substitution (NDIS) approach for the experimental determination of ion coordination numbers in systems involving both halide anions and oxyanions. We place particular emphasis on the frequently overlooked ion-pairing phenomenon, identify its microstructural signature in the neutron-weighted distribution functions, and suggest novel techniques to deal with either the estimation of the ion-pairing magnitude or the correction of its effects on the experimentally measured coordination numbers. We illustrate the underlying ideas by applying these new developments to the interpretation of four NDIS test-cases via molecular simulation, as convenient dry runs for the actual scattering experiments, for representative aqueous electrolyte solutions at ambient conditions involving metal halides and nitrates.
\end{abstract}

Keyword: Molecular simulation, aqueous electrolytes, ion pair association, neutron diffraction with isotopic substitution, heavy water, null-environments

\title{
I. INTRODUCTION
}

Researchers have early recognized that ion-water interactions are determining factors in the behavior of aqueous electrolyte solutions typically encountered in biological, geochemical, environmental, and industrial processes. Consequently, they have focused their attention on the properties of electrolyte solutions in terms of the underlying intermolecular forces and sought their interpretation in terms of experimentally measured structural features via diffraction approaches ${ }^{1}$. While ion hydration has been historically studied in a thermodynamic context whose description of the observables was restricted to concentration domains where they obey simple limiting laws (e.g., Debye-Huckel ${ }^{2}$ ), the lack of microscopic input hindered the development of accurate molecular interpretations. For a long time the "missing link" was the lack of a 
clear and unambiguous connection between the hydration process and its corresponding thermodynamics, until Ben-Naim introduced a generalized stepwise and clever approach that allowed identifying the underlying statistical mechanical basis and its relevant hydration thermodynamic quantities ${ }^{3-4}$

From a modeling perspective the hydration process can be thought as a local density perturbation (or solute-induced changes of the solvent environment toward the formation of the hydration microstructure) caused by the introduction of solute species into the aqueous environment that plays a fundamental role in determining the thermophysical behavior of solutions ${ }^{5-6}$. This local perturbation arises from the difference of strength (or interaction asymmetry) between the water-water and the solutewater interactions whose macroscopic outcome is always the manifestation of solution nonideality ${ }^{7}$.

The microstructural changes of water around ionic species in aqueous electrolyte solutions are the unavoidable common denominator for all hydration-related phenomena including ion mobility and conductivity ${ }^{8-9}$, solvent electrostriction and dielectric saturation ${ }^{10-11}$, salt solubility and ion speciation ${ }^{12-13}$, electrodic processes ${ }^{14}$, saltingin/out in mixed-solvent electrolytes ${ }^{15}$, as well as protein denaturing and folding ${ }^{16-17}$. This commonality is not accidental but the manifestation of fundamental links between the microstructure of the system, described in terms of (either radial or spatial) distributions functions, and relevant macroscopic properties ${ }^{18}$ that might become the basis for macroscopic modeling and useful engineering correlations ${ }^{19-20}$. In fact, there are basically two available exact formalisms able to provide rigorous (i.e., cause-effect) connections between the microscopic details of the system and their macroscopic properties, in chronological order, McMillan-Mayer (MM) ${ }^{21}$ and Kirkwood-Buff (KB) fluctuation formalisms ${ }^{18}$.

While the two theoretical approaches are based on configurational integrals over grand canonical correlation functions, to date their use as means of guiding the interpretation and modeling of electrolyte solutions has been contrastingly different. As discussed recently by Vafaei et al. ${ }^{22}$ who are attempting to change that trend, the observed usage disparity between the two formalisms might be in part because the original MM manuscript is "hard to read" and the formalism reduces the analysis of the 
solution to a solute-only form, i.e., where the solvent degrees of freedom in the corresponding partition function are integrated out. Moreover, because the MM formalism delivers thermodynamic properties as power series of the solute concentrations, whose higher order coefficients are difficult to interpret, the formalism has been useful only for dilute solutions ${ }^{23-25}$. In contrast, the KB formalism involves all species correlation functions where certain combinations of their configurational integrals (i.e., the so-called $\mathrm{KB}$ integrals) can be related directly to macroscopic properties including the solution compressibility, the ion osmotic susceptibilities and corresponding partial molar volumes ${ }^{6,26}$.

An immediate practical consequence of such links between microscopic and macroscopic behavior has been the proliferation of the use of coordination (or hydration for that matter) numbers as descriptors of hydration strength or model parameters in the development of macroscopic correlators ${ }^{27-31}$. In fact, the conventional view of the structure of electrolyte solutions has been shaped by the schematic description of hydration by Frank and Wen ${ }^{32}$ and Gurney ${ }^{33}$ based on concentric water shells around the ions — innermost or primary, secondary, and outermost — to interpret ion hydration in terms of structure-breakers (or chaotropos, i.e., they weaken the hydrogen bonding network) and structure makers (or kosmotropos, i.e., they strengthen the hydrogen bonding network $)^{34}$. With this scheme came the challenge of an adequate metric to discriminate between the two structures according to experimentally measured properties such as the dielectric constant of water in the electrolyte solution and the entropy of ion solvation ${ }^{35}$. This picture also introduced the idea of the ion-water coordination ( $a k a$ hydration number) as the estimated number of water molecules associated with the ion under consideration, a concept that simplified the interpretation and modeling of aqueous electrolyte systems ${ }^{14,36}$.

The ion-water coordination is obviously a natural description for the local water environment around an ion yet, its determination becomes strongly dependent on the methodology of measurement and frequently they become adjustable parameters for modeling purposes ${ }^{8,14,35,37-38}$. Moreover, even though the concept of hydration numbers might be a rather deficient one ${ }^{39}$ — whose experimental determination by mobility, compressibility, dielectric measurements, and diffraction experiments provides a wide 
spread set of results ${ }^{40-42}$ - and oftentimes differentiated from the coordination numbers (for a lengthy discussion see Chapter 29 of Ref. 35), its frequent usage highlights the need for more direct ways to probe the water environment around the ions in solution.

In what follows we will discuss relevant issues underlying the subject of interest to fulfill the premise behind the goal of this manuscript; however, due to obvious scope and length constraints we will focus on aspects that are close to our own past and current research activities. In the next sections we address the microstructural analysis of simple aqueous electrolytes and present a detailed account of the fundamentals underlying the NDIS approach for the experimental determination of ion coordination numbers. Then, we discuss the frequently overlooked ion-pairing phenomenon, identify its microstructural signature in the neutron-weighted distribution functions, and propose novel techniques to deal either with the determination of ion-pairing or the correction of its effects on the measured coordination numbers. Finally, we apply these developments to the interpretation of NDIS outputs from molecular simulation of representative aqueous electrolyte solutions at ambient conditions involving metal halides and nitrates. We close the manuscript with a brief discussion and relevant final remarks.

\section{MICROSTRUCTURE OF SIMPLE AQUEOUS ELECTROLYTES}

In the last four decades we have witnessed the significant investment of effort toward the development of tools for the extraction and collection of structural information of aqueous electrolyte solutions from scattering experiments, mainly, by xray and neutron scattering methods ${ }^{43-49}$. In particular, the ability of manipulating the isotopic composition of a targeted ion makes NDIS a powerful tool to extract structural information about the electrolyte solution in terms of ion-water (by the first-order difference method) ${ }^{43}$ and the ion-ion (by the second-order difference method) correlations ${ }^{50}$. This approach is made possible because the neutron scattering length is isotope specific, and consequently, neutrons can distinguish different isotopic forms of the same elements, a feature that provides the opportunity to develop novel experimental schemes, especially when an element exhibits isotopic forms, , whose neutron coherent scattering lengths $b$ are of opposite signs. In fact, the most prominent of them is given by the ${ }^{1} H /{ }^{2} H(a k a H / D)$ isotopic substitution with $b_{H}=3.74 \mathrm{fm}$ and $b_{D}=6.67 \mathrm{fm}^{51}$, 
where this condition makes possible the tuning of the global coherent scattering of the hydrogen element to become transparent to neutron, e.g., in the case of "null water" or "semi-transparent" environments to be discussed in detail below ${ }^{52}$.

In addition to ${ }^{1} H /{ }^{2} H$, there are other elements with isotopic variations whose coherent scattering lengths exhibit opposite signs, including ${ }^{6} \mathrm{Li} /{ }^{7} \mathrm{Li},{ }^{46} \mathrm{Ti} /{ }^{48} \mathrm{Ti}$, ${ }^{50} \mathrm{~V} /{ }^{51} \mathrm{~V},{ }^{52} \mathrm{Cr} /{ }^{53} \mathrm{Cr},{ }^{58} \mathrm{Ni} /{ }^{62} \mathrm{Ni},{ }^{112} \mathrm{Cd} /{ }^{113} \mathrm{Cd},{ }^{123} \mathrm{Te} /{ }^{130} \mathrm{Te},{ }^{130} \mathrm{Ba} /{ }^{138} \mathrm{Ba},{ }^{149} \mathrm{Sm} /{ }^{147} \mathrm{Sm}$, and ${ }^{184} \mathrm{~W} /{ }^{186} \mathrm{~W}$. Below we will discuss how this feature can in principle be used to "erase" some correlation peaks, and consequently, avoid potential peak overlapping that prevents the proper interpretation of water-ion coordination (a full account of this novel approach is given elsewhere ${ }^{53}$ ). In fact, peak overlapping is a phenomenon of common occurrence in aqueous electrolytes, a signature of anion-cation hydration shell penetration (aka ion pairing) that can affect the interpretation of the shape, location, and deconvolution of scattering peaks ${ }^{54-55}$.

NDIS of aqueous electrolyte solutions typically involves heavy rather than light water, to overcome the large effects of incoherent scattering of the neutrons by the protons ${ }^{56}$. However, and provided that the significantly larger (than those for heavy water) corrections for incoherent scattering can be properly applied ${ }^{57}$, the feasibility of using light water and light-heavy water mixtures as aqueous environments increases the power of this tool for the extraction of partial structural factors, and consequently, ion coordination information (vide infra).

\section{a) Fundamentals underlying NDIS for the determination of ion coordination}

In order to set up the foundations for the ensuing discussion here we identify the link between the measured quantities and the targeted microstructures (e.g., ion coordination environment), and discuss the challenges behind the meaningful interpretation of the experimental evidence, including peak overlapping as the signature of ion-pair formation ${ }^{58-59}$. For that purpose we focus on the useful portion of the neutron scattering differential cross section of amorphous aqueous samples, $d / d$, that 
provides the desired information on their microstructure, i.e., the total structure factor $F(q)$ defined as ${ }^{46}$,

$$
F(k)=\quad{ }_{i} \quad c_{j} c_{j} b_{i} b_{j}\left(S_{i j}(k) 1\right)
$$

where $k=(4 /) \sin (/ 2)$ represents the magnitude of the scattering vector as a function of the wavelength of the incident neutrons and the scattering angle,$c_{i}$ and $b_{i}$ denote the atomic fraction and the coherent neutron scattering length of atomic species $i$, respectively, and $S_{i j}(k)$ is the partial structure factor describing the correlation between atoms of types $i$ and $j$, i.e.,

$$
S_{i j}(k)=1+(4 \quad / k) \int_{0}^{\infty}\left[g_{i j}(r) 1\right] r \sin (k r) d r
$$

where and $g_{i j}(r)$ are the atomic number density of the solution and the radial pair distribution function for $i j$ interactions.

In order to extract $F(k)$ from the experimentally measured neutron scattering differential cross section $d / d$ (or mean intensity), i.e.,

$$
F(k)=d / d \quad \sum_{i} c_{i}{ }_{i}{ }_{i}^{\text {total }} / 4 \quad \text { relevant corrections }
$$

we need to subtract the total (coherent plus incoherent) cross section ${ }_{i}^{t o t a l}$ for all scattering centers $i$, and then correct for inelastic (Placzek corrections ${ }^{60}$ ) and multiple scattering, as well as for sample and container adsorption ${ }^{61}$. While these corrections are not trivial tasks ${ }^{62}$, for the sake of argument we will assume that the instrument scientist behind the experiments will deal with them properly to be able to determine $F(k)$ accurately from the $d / d$ raw data.

Considering the challenges underlying the NDIS experiments including the processing of resulting raw data, and the fact that there is no need for any of the above corrections for the atomistic simulated $F(k)$ or its real space counterpart $G(r)$, it becomes extremely useful to develop molecular simulation "dry runs" of NDIS experiments to determine the location of relevant diffraction peaks, the presence of peak 
overlapping, and ultimately to facilitate the interpretation of the experimental evidence. In fact, this strategy provides the unmatched opportunity to assess the adequacy of neutron-weighted distribution function methodologies for the determination of species coordination in aqueous environments, to identify the presence of ion pairing, and to isolate its effect on the outcome of coordination calculations, regardless of the actual type and nature of the underlying intermolecular forces ${ }^{63}$.

From the simulation side, it is more advantageous to deal with the neutronweighted real space distribution function $G(r)$ rather than the structural factor $F(k)$, i.e., its Fourier transform,

$$
G(r)=\quad i_{j} c_{i} c_{j} b_{i} b_{j}\left(g_{i j}(r) 1\right)
$$

where $g_{i j}(r)$ is the radial pair distribution functions for the $i j$ pair interactions that results from the corresponding partial structural factor $S_{i j}(k)$ as follows,

$$
g_{i j}(r)=1+\left(\begin{array}{lll}
1 / 2 & 2 & r
\end{array}\right) \int_{0}^{\infty}\left[S_{i j}(k) \quad 1\right] k \sin (k r) d k
$$

Therefore, for a simple aqueous electrolyte solution involving an $M^{+} X$ type salt, $G(r)$ will comprise ten different pair correlations, with the largest contribution coming from the (light or heavy) water-water interactions, i.e., from either $g_{O H}(r)$ and $g_{H H}(r)$ or from $g_{O D}(r)$ and $g_{D D}(r)$.

Obviously, since our main goal is to determine the ion-water coordination, we need to decouple the most relevant $g_{i j}(r)$ 's from Eqn. (4), in particular, the overwhelming background contribution from water-water correlations to be able to extract some meaningful structural information concerning water around the ions. For that purpose we invoke the first-order difference method introduced by Soper et al. ${ }^{43}$, a technique that comprises two diffraction experiments, involving two identical solutions except for the different ( , ) isotopic states of the ion under study. At these conditions, and under the assumption of isomorphic structures upon isotopic substitution (i.e., species correlation functions are independent of the isotopic substitution ${ }^{64}$ ) such that we are able 
to subtract the water-water distribution functions from the total neutron-weighted distribution functions, i.e.,

$$
\begin{aligned}
& G_{I}^{\text {solv }}(r)=G_{I}^{\text {solv }}(r) \quad G_{I}^{\text {solv }}(r) \quad(\neq \quad \equiv \text { isotopic species }) \\
& =A_{I}\left(g_{O I}(r) 1\right)+B_{I}\left(g_{D I}(r) 1\right)+C_{I}\left(g_{H I}(r) 1\right)+ \\
& D_{I}\left(g_{C I}(r) 1\right)+E_{I}\left(g_{I I}(r) 1\right)
\end{aligned}
$$

In Eqn. (6) we use the superscript solv to represent the aqueous environment so that solv $(l w, h w, n w)$ will identify it as being light-, heavy-, or null-water, respectively (obviously we might also have a non-null light-heavy water mixture, though we will not discuss it here). Moreover, the subscripts $I, C, D, H$, and $O$ denote ion (either $X$ or $M^{+}$), counter ion, deuterium, hydrogen, and oxygen sites, respectively. Note that we purposely differentiate the prefactors $B_{I}$ and $C_{I}$, even though we adopted the isomorphic condition $g_{D I}(r)=g_{H I}(r)$, to highlight the power behind the manipulation of the aqueous environment to extract microstructural information (vide infra). The five prefactors in

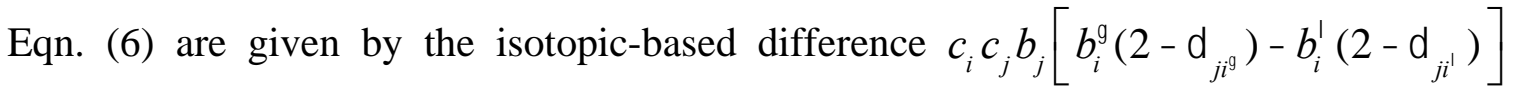
where we make explicit the isotopic dependence through the corresponding coherent neutron scattering lengths ( $b_{i}^{l}$ for $l=, \quad$ ) and invoke Kronecker delta $j_{j l}^{l}$ for the pair of isotopic species.

Oftentimes it is more useful to work with the normalized version of Eqn. (6) because it allows visualizing the relative contributions of the five terms, i.e.,

$$
\begin{aligned}
G_{I}^{\text {solv }, \text { norm }}(r)= & {\left[A_{I} g_{O I}(r)+B_{I} g_{D I}(r)+C_{I} g_{H I}(r)+\right.} \\
& \left.D_{I} g_{C I}(r)+E_{I} g_{I I}(r)\right] /{ }_{I}^{\text {solv }}
\end{aligned}
$$

where ${ }_{I}^{\text {solv }}=A_{I}+B_{I}+C_{I}+D_{I}+E_{I}$, so that $G_{I}^{\text {solv, norm }}(r \rightarrow$ large $)=1$. Regardless of the choice, Eqns. (6)-(7) still comprise five linearly independent unknowns suggesting that we would need at least five independent experiments to be able to extract the sought microscopic information. This information would be captured by a portion of either $G_{I}^{\text {solv }}\left(r_{L}<r<r_{U}\right)$ or $G_{I}^{\text {solv,norm }}\left(r_{L}<r<r_{U}\right)$ for the radial region where the relevant 
correlation peak is located, under the proviso that the peak comprises the contribution of only one type of pair interactions, i.e., absence of peak overlapping.

In order to comprehend the main issues behind the $G_{I}^{\text {solv }}(r)$ (or $G_{I}^{\text {solv,norm }}(r)$ for that matter) profiles we reproduce in Figures 1-2 the experimental curves for $\mathrm{Ni}^{+2}$ in a heavy water $\mathrm{NiCl}_{2}$ solution, and for $\mathrm{Cl}$ in a heavy water $\mathrm{LiCl}$ solution, respectively, taken with permission from of the Royal Society ${ }^{65}$. The main features we need to highlight are the identities of the pair correlations conforming the main two peaks, in particular we should note that $G_{M}^{\text {solv }}(r)$ exhibits an $O M$ peak followed by the corresponding $H \quad M$ (or $D M$ ) peak, within the first water-cation hydration shell, which are usually pretty much decoupled. In contrast, $G_{X}^{\text {solv }}(r)$ displays first an $H \quad X$ (or $D \quad X$ ) peak followed the overlapping between the $O \quad X$ and the second $H \quad X$ correlation corresponding to the nearest coordinating water molecules. Moreover, depending on the ion's charge density and the solution's ionic strength these peaks might comprise additional contributions due to their partial overlapping with the $M^{+} X$ pair correlations whose first peak (i.e., the CIP configuration) usually falls between those of corresponding to the first coordination of the two ions.

From a hydration viewpoint, the $M^{v+} \cdots X^{v-}$ pair formation results from the penetration of the anion into the cation hydration shells, leads to overlapping of the $M^{+} \quad X \quad$ with the $H \quad M^{+}$or $H \quad X \quad$ correlations, and depending on the extent of the overlap it might appear as a distortion of the normal shape of the second peaks of $G_{M}^{h w}(r)$, and the first peaks of the $G_{X}^{h w}(r)$ (vide infra, Figures 5-8). This distortion in the main peaks of $G_{I}^{h w}(r)$ can be usually subtle, and consequently, a visual inspection of the shape of either $G_{M}^{h w}(r)$ or $G_{X}^{h w}(r)$ might not provide any hint on the $M^{v+} \cdots X^{v-}$ presence.

The next step has been usually to assume negligibly small contributions to either $G_{I}^{\text {solv }}\left(r_{L}<r<r_{U}\right)$ or $G_{I}^{\text {solv,norm }}\left(r_{L}<r<r_{U}\right)$ from the ion-ion and the ion-counterion interactions ${ }^{66-70}$, i.e., $\left[\left|D_{I}\right|,\left|E_{I}\right|\right] \square\left[\left|A_{I}\right|,\left|B_{I}\right|,\left|C_{I}\right|\right]$. Note however that the actual contributions of the $\left[D_{I}, E_{I}\right]$ terms to either $G_{I}^{\text {solv }}\left(r_{L}<r<r_{U}\right)$ or $G_{I}^{\text {solv,norm }}\left(r_{L}<r<r_{U}\right)$ 
comprise the factors $\left[g_{C I}(r), g_{I I}(r)\right]$ so that for very soluble salts, where increasing ionic strength induce ion-pair formation, neither the coefficients $\left[D_{I}, E_{I}\right]$ nor the correlation functions $\left[g_{C I}(r), g_{I I}(r)\right]$ may be considered negligible as already shown elsewhere ${ }^{58,71}$. This scenario highlights several pending issues regarding the ability of current methods of processing NDIS data to extract reliable ion-water coordination numbers involving $M^{+} X$ aqueous systems that exhibit ion-pair formation, a phenomenon that interferes with the interpretation of either $G_{I}^{\text {solv }}\left(r_{L}<r<r_{U}\right)$ or $G_{I}^{\text {solv,norm }}\left(r_{L}<r<r_{U}\right)$ peaks. In fact, while often overlooked, the relevant issues here are: (a) how to confirm whether the system exhibits ion pairing, and if present, (b) how to decouple the peak overlapping and/or determine the ion-pairing effect on the "measured" ion-water coordination.

This is precisely the context where molecular-based simulation becomes a powerful tool to assist and guide the accurate interpretation of the scattering raw data, because simulation provides the two ends of the analysis and their rigorous connection, i.e., not only the set of correlation functions for all pair interactions, but also the corresponding neutron weighted distribution functions. In other words, simulation offers answers to precisely defined questions as a direct path to the unambiguous test of consistency and accuracy for the methods and conjectures used in the extraction of structural information from NDIS experiments.

To illustrate this issue, let us start with the statistical mechanical definition of the coordination number as the number of water's site surrounding a central ion $I$ within a hydration shell of radius $r_{s}$, i.e.,

$$
\bar{n}_{I}\left(r_{s}\right)=4 \quad{ }_{0}^{r_{s}} g_{I}(r) r^{2} d r
$$

where $=H, O$ and $r_{s}$ typically locates the first valley of the radial distribution function $g_{I}(r)$. Thus, if we had a way to determine the radial distribution function $g_{I}(r)$ at the conditions of the system of interest (in practice only possible from simulation or theory), then we would be able to provide an accurate estimate of the corresponding water coordination around the chosen ion according to Eqn. (8). Obviously, this is not possible from scattering experiments even though we can "dig up" 
the information from the radial integration of either $G_{I}^{\text {solv }}\left(r_{L}<r<r_{U}\right)$ or $G_{I}^{\text {solv,norm }}\left(r_{L}<r<r_{U}\right)$ peaks as long as we can ensure the compliance of the indicated constrains (a)-(b) (vide supra).

After having attained the neutron-weighted radial distribution functions $G_{I}^{\text {solv,norm }}(r)$ or $\quad G_{I}^{\text {solv }}(r)$ from some NDIS experiments, and after invoking Eqns. (6)-(7) we can rewrite Eqn. (8) as follows,

$$
\begin{aligned}
& \bar{n}_{I}\left(r_{L}, r_{U}\right)=(4 \quad / I) \int_{r_{L}}^{r_{U}}\left(G_{I}^{\text {solv }}(r)+{ }_{I}^{\text {solv }}\right) r^{2} d r \\
& =\left(\begin{array}{ll}
4 & { }_{I}^{\text {solv }} / I
\end{array}\right) \int_{r_{L}}^{r_{U}} G_{I}^{\text {solv,norm }}(r) r^{2} d r
\end{aligned}
$$

with $I=A_{I}, B_{I}, C_{I}, D_{I}, E_{I}$, and $=O, H, D, I$, where we implicitly assume that either $G_{I}^{\text {solv }}\left(r_{L}<r<r_{U}\right)$ or $G_{I}^{\text {solv,norm }}\left(r_{L}<r<r_{U}\right)$ exhibits well-defined and resolved peaks, i.e., the integration over for the interval $\left(r_{L}, r_{U}\right)$ would comprise only $I$ pair correlations. Thus, when these assumptions are not obeyed the "measured" coordination numbers would misrepresent the actual water environment around the ion. This is typically the case encountered in the study of the water's hydrogen environment around simple halide anions (alkaline cations) where we expect to find partial overlapping of the $H \quad X\left(\begin{array}{ll}H & M\end{array}\right)$ and the $X \quad M$ when the aqueous $M^{+} X$ exhibit contact ion-pair (CIP) configurations ${ }^{58,72-73}$.

Then, the question is how to detect the presence of anion-cation pairing, and how to correct Eqn. (9) for such occurrence. For the sake of argument let us assume that the solvent is heavy water, i.e., $C_{I}=0$, and the isotopic substitution is done on the anion, i.e., $(X / X)$. Under those conditions Eqn. (9) would read as follows,

$$
\bar{n}_{X}^{D}(\text { measured })=\left(\begin{array}{ccc}
4 & & { }^{h w} \\
& D & I
\end{array} / B_{X}\right) \int_{r_{L}}^{r_{U}} G_{X}^{h w, n o r m}(r) r^{2} d r
$$

where we "suspect" there is a potential peak overlapping within the interval $\left(r_{L}, r_{U}\right)$, i.e., $G_{X}^{h w, n o r m}\left(r_{L}<r<r_{U}\right) \quad\left[B_{I} g_{D I}(r)+D_{I} g_{C I}(r)\right] /{ }_{I}^{\text {solv }}$, from which it follows that, 


$$
\begin{aligned}
& \bar{n}_{X}^{D}(\text { measured })=\left(\begin{array}{ll}
4 & { }_{D} / B_{X}
\end{array}\right){ }_{r_{L}}^{r_{U}}\left(B_{X} g_{D X}(r)+D_{X} g_{M X}(r)\right) r^{2} d r
\end{aligned}
$$

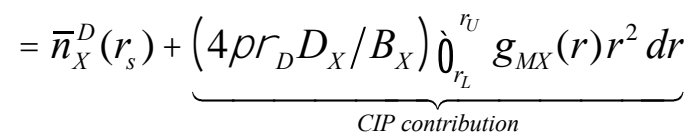

The CIP contribution to the "measured" water's hydrogen coordination of the anion, over the true value $\bar{n}_{X}^{D}\left(r_{s}\right)$, can be further simplified by invoking the identities of the coefficients $D_{X}$ and $B_{X}$, i.e.,

$$
\begin{aligned}
& \text { (4 } \left.{ }_{D} D_{X} / B_{X}\right){ }_{r_{L}}^{r_{U}} g_{M X}(r) r^{2} d r=\left(4{ }_{M} b_{M} / b_{D}\right){ }_{r_{L}}^{r_{U}} g_{M X}(r) r^{2} d r \\
& =\bar{n}_{X}^{M}\left(r_{s}\right)\left(b_{M} / b_{D}\right)
\end{aligned}
$$

where, by analogy to Eqn. (8), $\bar{n}_{X}^{M}\left(r_{s}\right)$ represents the cation coordination of the anion. Likewise, the cation counterpart to Eqn. (11) becomes,

$$
\begin{aligned}
\bar{n}_{M}^{D}(\text { measured }) & =\left(4 \pi \rho_{D} / B_{M}\right) \int_{r_{L}}^{r_{U}}\left(B_{M} g_{D M}(r)+D_{M} g_{M X}(r)\right) r^{2} d r \\
& =\bar{n}_{M}^{D}\left(r_{s}\right)+\underbrace{\left(4 \pi \rho_{D} D_{M} / B_{M}\right) \int_{r_{L}}^{r_{U}} g_{M X}(r) r^{2} d r}_{\text {CIP contribution }}
\end{aligned}
$$

where after interpreting its meaning, the $C I P$ contribution reduces to the simpler form,

$$
\begin{aligned}
\left(4{ }_{D} D_{M} / B_{M}\right){ }_{r_{L}}^{r_{U}} g_{M X}(r) r^{2} d r & =\left(\begin{array}{ll}
4 & { }_{X} b_{X} / b_{D}
\end{array}\right){ }_{r_{L}}^{r_{U}} g_{M X}(r) r^{2} d r \\
& =\bar{n}_{M}^{X}\left(r_{s}\right)\left(b_{X} / b_{D}\right)
\end{aligned}
$$

with $\bar{n}_{M}^{X}\left(r_{s}\right)=\left(c_{X} / c_{M}\right) \bar{n}_{X}^{M}\left(r_{s}\right)$ according to the solution composition.

In summary, in order to attain the true water's hydrogen coordination of the anion $\bar{n}_{X}^{D}\left(r_{s}\right)$ (cation $\left.\bar{n}_{M}^{D}\left(r_{s}\right)\right)$ from the corresponding $\bar{n}_{X}^{D}$ (measured) $\left(\bar{n}_{M}^{D}(\right.$ measured $\left.)\right)$ we need also to estimate the extent of the contact anion-cation coordination, i.e., either $\bar{n}_{X}^{M}\left(r_{s}\right)$ or $\bar{n}_{M}^{X}\left(r_{s}\right)$. This is a situation where the manipulation of the isotopic composition of the 
aqueous environment becomes most powerful, in particular when $B_{I}=C_{I}$ in Eqn. (7), i.e., for $c_{D} / c_{H}=b_{H} / b_{D} \quad 0.561$ according to the scattering lengths given by Sears ${ }^{51}$. This is the so-called null-water environment that, due to the absence of scattering by the water's hydrogens, in principle allows performing NDIS experiments able to isolate the contact anion-cation coordination contribution to either the $\bar{n}_{X}^{D}$ (measured) or $\bar{n}_{M}^{D}$ (measured). In fact, from Eqn. (7) and for the null-environment we have that,

$$
G_{I}^{n w, n o r m}(r)=\left[A_{I} g_{O I}(r)+D_{I} g_{C I}(r)+E_{I} g_{I I}(r)\right] /{ }_{I}^{n w}
$$

with ${ }_{I}^{n w}=A_{I}+D_{I}+E_{I}$, and after invoking the definition of $\bar{n}_{X}^{M}\left(r_{s}\right)$ we find that,

$$
\begin{aligned}
\bar{n}_{X}^{M}\left(r_{s}\right) & =4 \quad{ }_{M} \int_{r_{L}}^{r_{U}} g_{M X}(r) r^{2} d r \\
& =\left(\begin{array}{lll}
4 & M & { }_{M}^{n w}
\end{array} / D_{M}\right) \int_{r_{L}}^{r_{U}} G_{M}^{n w, n o r m}(r) r^{2} d r
\end{aligned}
$$

where we have performed the first-order difference over the cation isotopes, $I=M$. Alternatively, if the first order difference is over the substituted anion, $I=X$, then,

$$
\begin{aligned}
& \bar{n}_{M}^{X}\left(r_{s}\right)=4 \quad{ }_{X} \int_{r_{L}}^{r_{U}} g_{M X}(r) r^{2} d r \\
& =\left(\begin{array}{lll}
4 & X & { }_{X}^{n w}
\end{array} / D_{X}\right) \int_{r_{L}}^{r_{U}} G_{X}^{n w, n o r m}(r) r^{2} d r
\end{aligned}
$$

so that again $\bar{n}_{M}^{X}\left(r_{s}\right)=\left(c_{X} / c_{M}\right) \bar{n}_{X}^{M}\left(r_{s}\right)$, the quantities required to complete the calculation of $\bar{n}_{X}^{D}\left(r_{s}\right)$ and $\bar{n}_{M}^{D}\left(r_{s}\right)$ in Eqns. (12) and (13), respectively.

To illustrate these developments, in what follows we present a few representative case studies of aqueous electrolytes involving either simple anions (chlorides) or more complex oxyanions (nitrates) and discuss the results in the context of experimental data and $a b$ initio results whenever available. In terms of notation we will be using the sub/super-script $D$ interchangeable with $H$ or $H_{W}$, i.e., to identify the atomic species water hydrogen in microstructural/correlation/distribution functions. However note that 
$D$ and $H$ will not be interchangeable when referring to the scattering length of the isotopic species.

\section{b) Illustration of the methodology for model systems involving monoatomic anions}

\section{Case Study 1: Ion hydration in aqueous $\mathrm{CaCl}_{2}$ solutions}

Calcium chloride is an important component frequently found in geological formations and in aqueous solutions involved in industrial processes. For that reason, its aqueous solutions have been the focus of extensive thermodynamic ${ }^{74-80}$ and structural studies by neutron scattering ${ }^{73,81-85}$, X-ray diffraction (XRD) ${ }^{86-93}$, extended X-ray absorption fine structure (EXAFS) ${ }^{93-97}$, electric conductance ${ }^{98-99}$, and molecular simulation ${ }^{90,100-106}$.

Despite the wealth of experimental and simulation studies of aqueous $\mathrm{CaCl}_{2}$ there is a persistent uncertainty about the nature of the $\mathrm{Ca}^{+2} \cdots \mathrm{Cl}$ pair formation, i.e., $C I P$ versus solvent-shared (SShIP) configurations, and how ionic strength and state conditions affect its occurrence. For example, after reviewing most experimental datasets for the thermodynamic properties of aqueous $\mathrm{CaCl}_{2}$, Rard and Clegg ${ }^{77}$ concluded that the standard Pitzer equations ${ }^{107}$ and their empirically extended expressions were able to describe the behavior of $\mathrm{CaCl}_{2}$ over the entire molality range only when they included explicitly the $\mathrm{CaCl}_{(a q)}^{+}$pair formation. However, Pitzer et al. ${ }^{78}$ later argued that Rard and Clegg's analysis did not settle the argument on the need to account for speciation, in that the impact of the inaccuracies in the association constant $\mathrm{K}^{o}\left[\mathrm{CaCl}^{+}\right]$translates into the incorporation of higher virial terms. This scenario highlights the need for a more direct way to assess the presence of ion speciation, e.g., in terms of its microstructural signature via scattering techniques.

On the one hand, early wide angle X-ray scattering (WAXS) experiments on concentrated $\mathrm{CaCl}_{2}$ solutions by Yamaguchi et al. ${ }^{89}$ and their re-interpretation of previous data from Caminiti et al. ${ }^{88}$ suggested the presence of CIP configuration at $\sim 2.74 \AA$ and a SShIP configuration at $\sim 4.8 \AA$ (see brief discussion on this reinterpretation previous to their Table II). More recent experimental studies targeting 
specifically this hydration phenomenon provided support to Yamaguchi et al.'s findings. For example, the Hungarian team led by Gabor Pálinkás ${ }^{73,92}$ performed XRD of aqueous $\mathrm{CaCl}_{2}$ in the $16 \mathrm{M}$ range at ambient conditions and concluded that about $4 \mathrm{M}$ the solution forms SShIP at $4.85 \AA$ together with $C I P$ at $2.72 .75 \AA$. The appearance of ion pairing was accompanied by a significant decrease of the water coordination of $\mathrm{Ca}^{+2}$, $\bar{n}_{C a}^{O}\left(r_{s}\right)$, with ionic strength, a behavior that follows closely the simulated microstructural results of Chialvo and Simonson ${ }^{58}$ as illustrated in Figure 3. While XRD was unable to detect ion pairing below $4 M$, the ion-pair association above this concentration followed the trend found by simulation as illustrated in Figure 4.

On the other hand, Fulton et al. ${ }^{83}$ used X-ray absorption fine structure (XAFS) spectroscopy to probe the effects of concentration on the first coordination of aqueous $\mathrm{Ca}^{2+}$, and found no evidence for significant contact $\mathrm{Ca}^{+2} \cdots \mathrm{Cl}$ pairs according to the $\mathrm{Ca}$ K-edge EXAFS spectrum for a $6 \mathrm{~m} \mathrm{CaCl}_{2}$ (Figure 4 in Ref. 83), yet the hexahydrate melt, $6.29 \mathrm{~m}$ solution, exhibits an average of $1.2 \mathrm{Cl}$ ions coordinating the $\mathrm{Ca}^{2+}$ ion. More recently, Pham and Fulton ${ }^{108}$ revisited the subject and introduced a new method for their characterization through the simultaneous data refinement from both XAFS and from high-energy XRD spectroscopy, i.e., x-ray absorption/diffraction simultaneous refinement (XADSR). This analysis for the $6 \mathrm{~m}$ aqueous $\mathrm{CaCl}_{2}$ identified $\sim 0.26$ contact $\mathrm{Ca}^{+2} \cdots \mathrm{Cl}$ pairs $(\mathrm{CIP})$ separated by $\sim 2.71 \AA$, as well as $\sim 3.4$ solventshared $\mathrm{Ca}^{+2} \cdots \mathrm{Cl}$ pairs $(S S h I P)$ separated by $\sim 4.98 \AA$. These authors also highlighted the underlying challenges behind the XRD spectra for the $6 \mathrm{mCaCl}_{2}$ resulting from the potential contact $\mathrm{Ca}^{+2} \cdots \mathrm{Cl}$ pair peak convolution with those associated with the $\mathrm{O} O$ and $\mathrm{O} C \mathrm{Cl}$ correlation peaks (Figure $1 \mathrm{~b}$ of Ref. 108).

Interestingly, almost at the same time, two new publications revisited the analysis of the potential of mean force ( $P M F$ ) for the $\mathrm{Ca}^{+2} \cdots C l$ pair according to either $a b$ initio constrained dynamics simulations ${ }^{105}$, or classical simulation of explicit polarizable models for both water and the ions ${ }^{109}$, where the $\mathrm{Ca}^{+2} \ldots \mathrm{Cl}$ force fields were adjusted through high level electronic structure calculations targeting the optimized pair structure. While the two studies predict similar profiles for the SShIP region centered at $\sim 5.0 \AA$, they show significantly different behavior for the CIP region around $\sim 2.75 \AA$. In 
particular, Dang et al.'s simulations indicate the absence of CIP at least for $m<1.0$, while Timko et al.' s ab initio PMF profile highlights the formation of both CIP and SShIP configurations with similar strengths, where both outcomes contrast significantly with the predictions from classical simulations involving non-polarizable models (e.g., those based on Dang-Smith force fields ${ }^{101,110-111}$ ).

Although these contrasting $P M F$ profiles are not unusual predictions from nonpolarizable models of aqueous $\mathrm{CaCl}_{2}$ (e.g., compare earlier results from Guardia et al. ${ }^{100}$ against those from Dang-Smith force fields ${ }^{101}$ ), the recent results highlight the importance of polarization effects and their dependence with ionic strength. In fact, the recent Car-Parrinello molecular dynamics (CPMD) simulations of $2 \mathrm{~m}$ aqueous $\mathrm{CaCl}_{2}$ by Todorova et al. ${ }^{104}$ suggest the presence of stable CIP and SShIP configurations (note however that these results correspond to $T=320 \mathrm{~K}$ ), where the average dipole moment of the coordinating water is significantly affected by the coordination strength of the $\mathrm{Ca}^{+2}$.

The above state of affairs attests to difficulties encountered when interpreting the hydration behavior of ions according to tools that involve different sets of conjectures and approximations in linking the experimental output and the phenomenon/property under study. In what follows we illustrate the challenges encountered by the scattering practitioners when pursuing the extraction of microstructural information from NDIS on a $6.4 \mathrm{~m} \mathrm{CaCl}_{2}$ aqueous solution at ambient conditions involving either the ${ }^{n a t} \mathrm{Ca} /{ }^{44} \mathrm{Ca}$ or the ${ }^{n a t} \mathrm{Cl} /{ }^{37} \mathrm{Cl}$ first-order difference scheme for a particular isotopic form of water. For that purpose we performed molecular dynamics simulation of precisely defined models to extract the ten possible pair distribution functions, to determine the relevant first-order difference $G_{I}^{\text {solv,norm }}(r)$ as a means for the characterization of ion hydration behavior, and to identify the sources of uncertainties and the challenges behind the interpretation of scattering data in the presence of ion-pair formation/peak overlapping/convolution. For details on the simulations, please see the Supplementary Information document.

Peak convolution - the consequence of the interpenetration of anion and cation hydration shells resulting from the decrease of dielectric screening of their electrostatic interactions, in response to an increase of ionic strength, system temperature, or both - 
is precisely the scenario where null-water environments becomes most useful. For example, according to Figures 5-6 for a $6.4 \mathrm{~m} \mathrm{CaCl}_{2}$ in heavy- (top) and null-water (bottom), the contact $\mathrm{Ca}^{+2} \cdots \mathrm{Cl}$ pair contributions overlap significantly the $D_{I} \mathrm{Ca}$ contribution to $G_{\mathrm{Ca}}^{h w, n o r m}(r)$ and becomes sandwiched between the $\mathrm{D}_{I} \mathrm{Cl}$ and the compound $O_{I} \quad C l \oplus D_{I I} \quad C l$ contributions to $G_{C l}^{h w, n o r m}(r)$, where subscripts $I$ and $I I$ denote first and second hydration shell, respectively. The integration of either the first peak of $G_{C l}^{h w, n o r m}(r)$ to determine the coordination number $\bar{n}_{C l}^{D}$ (measured), Eqn. (10), or the second peak of $G_{C a}^{h w, n o r m}(r)$ to assess the coordination number $\bar{n}_{C a}^{D}$ (measured), Eqn. (13), will account for some contributions from the CIP as discussed previously by Chialvo and Simonson ${ }^{58}$ (vide infra). By switching to the null-water environment, at the same atomic compositions, we eliminate the $D_{I} \quad \mathrm{Cl}, D_{I} \quad \mathrm{Ca}$, and $D_{I I} \mathrm{Cl}$ peaks, making possible the isolation of the CIP contributions in $G_{C l}^{n w, n o r m}(r)$ and $G_{C a}^{n w, n o r m}(r)$ and the determination of the corresponding $\bar{n}_{C l}^{C a}\left(r_{s}\right)$ (Eqn. (16)) and $\bar{n}_{C a}^{C l}\left(r_{s}\right)$ (Eqn. (17)).

Table 1: First water coordination of ions in $6.4 \mathrm{~m} \mathrm{CaCl} 2$ heavy- and null-aqueous solutions according to the direct and the NDIS-based expressions

\begin{tabular}{|c|c|c|}
\hline pair interactions & direct integral $\left(r_{s}\right)^{(\mathrm{c})}$ & NDIS integral $\left(r_{s}\right)^{(\mathrm{d})}$ \\
\hline$O_{W} \quad \mathrm{Ca}$ & $4.8(2.75 \AA)$ & $4.75(2.67 \AA)^{(\mathrm{b})}$ \\
\hline$D \quad C a$ & $10.7(3.5 \AA)$ & $12.7(2.17 \AA, 3.5 \AA)^{(\mathrm{a})}$ \\
\hline$O_{W} \quad \mathrm{Cl}$ & $8.7(4.25 \AA)$ & -- \\
\hline$D \quad C l$ & $4.5(2.97 \AA)$ & $5.1(2.97 \AA)^{(a)}$ \\
\hline $\mathrm{Ca} \mathrm{Cl}$ & $1.9(4.0 \AA)$ & $1.8(2.97 \AA)^{(\mathrm{b}, \mathrm{e})}$ \\
\hline
\end{tabular}

${ }^{\text {(a) }}$ heavy-water; ${ }^{\text {(b) }}$ null-water ; ${ }^{(\mathrm{c})} \bar{n}_{b}^{a}\left(r_{s}\right)=4 \quad{ }_{a}^{r_{s}} g_{a b}(r) r^{2} d r$ (Eqn. (8));

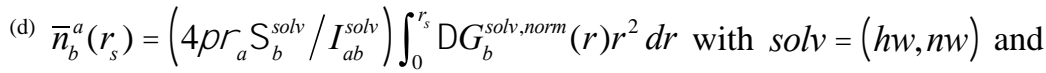
$I_{a b}^{\text {solv }}=\left(A_{b}^{\text {solv }}\right.$ if $a=O_{w} ; B_{b}^{\text {solv }}$ if $\left.a=D\right)$

${ }^{(\mathrm{e})}$ From Eqn. (16) $\bar{n}_{C a}^{C l}\left(r_{s}\right)=1.8$, while from Eqn. (17) $\bar{n}_{C l}^{C a}\left(r_{s}\right)=0.98$, i.e., $\bar{n}_{C a}^{C l}\left(r_{s}\right) \quad 2 \bar{n}_{C l}^{C a}\left(r_{s}\right)$

As clearly illustrated in Table 1, where we collect all calculated ion coordination numbers, the comparison between heavy-water NDIS-based quantities and the actual values (Eqn. (8)) for precisely the same system microstructure indicates significant 
differences. A simple inspection of the individual contributions to the corresponding first-order differences in Figures 5 and 6 reveals the presence of contributions from contact $\mathrm{Ca} \cdots \mathrm{Cl}$ pairs in $G_{C l}^{h w, n o r m}(r)$ and $G_{C a}^{h w, n o r m}(r)$, whose extent can be evaluated according to the previously derived expressions. In particular, note that the CIP coordination, $\bar{n}_{C a}^{C l}\left(r_{s}\right)$, contributions to $\bar{n}_{C a}^{D}\left(r_{s}\right)$ and $\bar{n}_{C l}^{D}\left(r_{s}\right)$ are modified by the prefactors $\left(b_{C l} / b_{D}\right) \quad 1.43$ and $\left(b_{C a} / b_{D}\right) \quad 0.70$, respectively. After correcting the NDIS-based coordination numbers for those CIP contributions, Eqns. (11)-(14), we attain the sought values that are now in agreement with the expected values from the direct integration of the corresponding radial distribution functions, Eqn. (8), as illustrated in Tables 2-3. For that purpose we have used the null-water NDIS-based $\bar{n}_{C a}^{C l}\left(r_{s}\right)$ and $\bar{n}_{C l}^{C a}\left(r_{s}\right)$ values according to Eqns. (16)-(17) (see also bottom of Figures 5 and 6).

Table 2: First coordination numbers of $\mathrm{Cl}$ in $6.4 \mathrm{~m} \mathrm{CaCl}_{2}$ aqueous solutions from integrals over $G_{C l}^{\text {solv, norm }}(r)$

(a) $\bar{n}_{C l}^{D}\left(r_{s}\right)=$\begin{tabular}{|c|c|c|}
\hline $\bar{n}_{C l}^{D}\left(\right.$ Eqn. 8), $r_{s}$ & $\bar{n}_{C l}^{D}\left(\right.$ Eqn. 10), $r_{L}, r_{U}$ & $\bar{n}_{C l}^{D}(\text { Eqns. 11-12) })^{(a)}$ \\
\hline $4.5,2.97 \AA$ & $5.1,0.0 \AA, 2.97 \AA$ & 4.4 \\
\hline
\end{tabular}

Table 3: First coordination numbers of $\mathrm{Ca}^{+2}$ in $6.4 \mathrm{mCaCl}_{2}$ aqueous solutions from integrals over $G_{\mathrm{Ca}}^{\text {solvorm }}(r)$

\begin{tabular}{|c|c|c|}
\hline $\bar{n}_{C a}^{D}\left(\right.$ Eqn. 8), $r_{s}$ & $\bar{n}_{C a}^{D}\left(\right.$ Eqn. 13), $r_{L}, r_{U}$ & $\bar{n}_{C a}^{D}\left(\right.$ Eqns. 13-14) $^{(a)}$ \\
\hline $10.7,3.5 \AA$ & $12.7,2.17 \AA, 3.5 \AA$ & 10.1 \\
\hline
\end{tabular}

(a) $\bar{n}_{C a}^{D}\left(r_{s}\right)=\bar{n}_{C a}^{D}($ measured $) \quad \bar{n}_{C a}^{C l}\left(r_{s}\right)\left(b_{C l} / b_{D}\right)$ where $\bar{n}_{C a}^{C l}\left(r_{s}\right) 1.8$ from Eqn. (17)

\section{Case Study 2: Ion hydration in aqueous $\mathrm{NiCl}_{2}$ solutions}

From the beginning, $\mathrm{NiCl}_{2}$ aqueous solutions have been the target of structural studies by neutron scattering ${ }^{67,112-114}$ and x-ray diffraction ${ }^{115-116}$. Unfortunately, the collected scattering information provides a conflicting picture of the hydration behavior (e.g., water coordination around ions) due to the potential occurrence of CIP and SShIP 
configurations, and calls for a re-examination of both the methods as well as the conclusions.

The proof for the presence of contact $\mathrm{Ni}^{+2} \cdots \mathrm{Cl}^{-}$pairs in aqueous for $\mathrm{NiCl}_{2}$ is as controversial as that for the $\mathrm{Ca}^{+2} \cdots \mathrm{Cl}^{-}$counterpart (vide supra), i.e., on the one hand Raman spectroscopy of concentrated $\mathrm{NiCl}_{2}$ aqueous solutions suggests the formation of $\mathrm{NiCl}_{4}{ }^{2}$ complexes ${ }^{117}$, on the other hand neither EXAFS ${ }^{118}$ nor X-ray adsorption nearedge structure (XANES) ${ }^{119}$ experiments found evidence of contact $\mathrm{Ni}^{+2} \cdots \mathrm{Cl}^{-}$pair configurations. Moreover, while earlier XRD evidence was rather inconclusive ${ }^{120}$, later studies by the same team provided evidence of the presence of $\mathrm{Ni}^{+2} \cdots \mathrm{Cl}^{-}$pair configurations by changing the $\mathrm{Ni}^{2+} / \mathrm{Cl}$ ratio through the addition of $\mathrm{LiCl}$ to the system. These authors concluded that the methodology underlying the processing of the XRD data might not always be able to detect a small number of contact anion-cation pairs ${ }^{121}$, a conclusion shared also by the work of Licheri et al. ${ }^{122}$. More recent XRD work by Waizumi et al. ${ }^{123}$ confirms the formation of contact $\mathrm{Ni}^{+2} \cdots \mathrm{Cl}^{-}$pair configurations, separated by $\square 2.37 \AA$, in aqueous $\mathrm{NiCl}_{2}$ within the concentration range $4.57 c(M) 5.47$ (i.e., $5.05 m 6.15$ along the saturation line) resulting in a $0.4 n_{\mathrm{NiCl}} 1.0$ range for the number of $\mathrm{Ni}^{+2} \cdots \mathrm{Cl}^{-}$interactions according to their notation. Interestingly, nuclear magnetic resonance (NMR) studies by Weingärtner et al. on $1.0 \mathrm{~m} \mathrm{NiCl}_{2}$ aqueous solutions found a coordination range of $0.14 \bar{n}_{\mathrm{Cl}}^{\mathrm{Ni}}\left(r_{s}\right) \quad 0.22$, depending on the "mechanism of ligand exchange" 124 , that translates into $0.62 \bar{n}_{C l}^{N i}\left(r_{s}\right) \quad 1.0$ at $4.4 m$ according to their extrapolations. To be fair we should note that the accuracy of NMR results might be susceptible to significant uncertainties originating in the large number of correlated parameters required in the fitting of relaxation data for the extraction of the degree of $\mathrm{Ni}^{+2} \cdots \mathrm{Cl}^{-}$association. Yet, as highlighted by Weingärtner et al. ${ }^{124}$ "there is no obvious explanation why nuclear magnetic relaxation and neutron scattering results should be very different, since both of them observe processes in the first coordination sphere fairly directly" we agree with Weingärtner et al.'s concern, and below we provide an explanation for such a dichotomy. Additional support for the existence of contact $\mathrm{Ni}^{+2} \cdots \mathrm{Cl}^{-}$pairs in $\mathrm{NiCl}_{2}$ aqueous 
solutions comes from recent far-infrared (FIR) spectroscopy studies of their hydration dynamics by Sharma et al. ${ }^{125}$ who determined ion-pair extinction spectra for $0.5 \quad m \quad 4.3$ that confirmed the CIP presence.

In order to address the Weingärtner et al. ${ }^{124}$ contention here we take advantage of the fact that both ions in this salt exhibit appropriate contrast for neutron scattering experiments, and as for the case of $\mathrm{H} / \mathrm{D},{ }^{n a t} \mathrm{Ni} /{ }^{62} \mathrm{Ni}$ also shows a change in the sign of the coherent scattering length with the possibility of a "null-nickel" environment by choosing the isotopic composition $c_{62}{ }_{N i} / c_{n a t}=\left(b_{n a t}{ }_{N i} / b_{6{ }^{2} N i}\right)$ 1.1839. This behavior, in principle, affords the opportunity to invoke a "null-nickel" approach that will provide a direct route to $\bar{n}_{C l}^{D}\left(r_{s}\right)$ from heavy-water NDIS experiments, avoiding the need for the $C I P$ correction to the measured coordination in heavy water environments ${ }^{53}$, Eqn. (11), whose evidence would be the correlation peak at $r_{s} \simeq 2.53 \AA$ as highlighted in Figures 78. Under this null-nickel environment the relevant distribution function $G_{C l}^{h w, n o r m}(r)$ reduces to a simpler one, i.e.,

$$
G_{C l, n N i}^{h w, n o r m}(r)=\left[A_{C l} g_{O C l}(r)+B_{C l} g_{D C l}(r)+E_{C l} g_{C l C l}(r)\right] /{ }_{C l, n N i}^{h w}
$$

as illustrated in Figure 9, where the subscript $n N i$ identifies the null-nickel condition and ${ }_{C l, n N i}^{h w}=A_{C l}+B_{C l}+E_{C l}$. Consequently, the coordination number $\bar{n}_{C l}^{D}\left(r_{s}\right)$ follows from the counterpart of Eqn. (9) for a heavy-water null-cation environment, i.e.,

$$
\bar{n}_{C l}^{D}\left(r_{s}\right)=\left(\begin{array}{lll}
4 & { }_{D} & h w \\
C l, n N i
\end{array} / B_{C l}\right) \int_{0}^{r_{s}} G_{C l, n N i}^{h w, n o r m}(r) r^{2} d r
$$

as given in Table 4 for a $3.9 \mathrm{~m} \mathrm{NiCl}_{2}$ solution in comparison with the corresponding values obtained from the corrected heavy-water estimations. Note that the experimental values quoted in the available literature are $5.5 \pm 0.4 \quad \bar{n}_{C l}^{D}\left(\right.$ Eqn. 10) $6.4 \pm 0.3^{67,69,126}$, but under the assumption $A_{C l}, B_{C l} C_{C l}, D_{C l}, E_{C l}{ }^{67,69-70}$ that precludes the participation of, and correction from, any ion-pair formation. Moreover, after extracting an NDIS-based coordination number $\bar{n}_{C l}^{D}\left(r_{s}\right)$ fully independent of the presence of $C I P$ contributions by 
means of a heavy-water null-cation environment, we can now check explicitly the subtle presence of, and contribution from, contact $\mathrm{Ni}^{+2} \cdots \mathrm{Cl}^{-}$pairs.

Table 4: First coordination numbers of $\mathrm{Cl}$ in $3.9 \mathrm{~m} \mathrm{NiCl}_{2}$ aqueous solutions from integrals over $G_{C l}^{\text {solv,norm }}(r)$ and $G_{C l, n N i}^{h w, n o m}(r)$

\begin{tabular}{|l|c||c|c|}
\hline \hline $\bar{n}_{C l}^{D}\left(\right.$ Eqn. 8), $r_{s}$ & $\bar{n}_{C l}^{D}\left(\right.$ Eqn. 10), $r_{L}, r_{U}$ & $\bar{n}_{C l}^{D}(\text { Eqns. 11-12) })^{(a)}$ & $\bar{n}_{C l}^{D}\left(\right.$ Eqn. 19), $r_{L}, r_{U}$ \\
\hline \hline $3.75,2.85 \AA$ & $5.4,0.0 \AA, 2.85 \AA$ & 3.70 & $3.75,0.0 \AA, 2.85 \AA$ \\
\hline (a) $\bar{n}_{C l}^{D}\left(r_{s}\right)=\bar{n}_{C l}^{D}($ measured $) \quad \bar{n}_{C l}^{N i}\left(r_{s}\right)\left(b_{N i} / b_{D}\right)$ where $\bar{n}_{C l}^{N i}\left(r_{s}\right) \quad 1.1$ from Eqn. (16)
\end{tabular}

Table 5: First coordination numbers of $\mathrm{Ni}^{+2}$ in $3.9 \mathrm{~m} \mathrm{NiCl}$ aqueous solutions from integrals over $G_{N i}^{\text {solv,norm }}(r)$

\begin{tabular}{|c|c|c|}
\hline $\bar{n}_{N i}^{D}\left(\right.$ Eqn. 8), $r_{s}$ & $\bar{n}_{N i}^{D}\left(\right.$ Eqn. 13), $r_{L}, r_{U}$ & $\bar{n}_{N i}^{D}$ (Eqns. 13-14) $^{(a)}$ \\
\hline $7.05,3.16 \AA$ & $10.2,2.30 \AA, 3.17 \AA$ & 7.04 \\
\hline
\end{tabular}

(a) $\bar{n}_{N i}^{D}\left(r_{s}\right)=\bar{n}_{N i}^{D}($ measured $) \quad \bar{n}_{N i}^{C l}\left(r_{s}\right)\left(b_{C l} / b_{D}\right)$ where $\bar{n}_{N i}^{C l}\left(r_{s}\right) \quad 2.2$ from Eqn. (17)

In fact, we can either invoke the null-water NDIS-based $\bar{n}_{N i}^{C l}\left(r_{s}\right)$ and $\bar{n}_{C l}^{N i}\left(r_{s}\right)$ values according to Eqns. (16)-(17) (see also bottom of Figures 7 and 8), or determined the $\bar{n}_{C l}^{D}($ measured $)$, Eqn. (10), from which we can extract $\bar{n}_{N i}^{C l}\left(r_{s}\right)$ as follows,

$$
\bar{n}_{N i}^{C l}\left(r_{s}\right)=\left[\bar{n}_{C l}^{D}(\text { measured }, h w) \quad \bar{n}_{C l}^{D}(h w, n N i)\right]\left(b_{D} / b_{N i}\right)
$$

where $\bar{n}_{C l}^{D}$ (measured, $\left.h w\right)$ and $\bar{n}_{C l}^{D}(h w, n N i)$ are given by Eqns. (10) and (19), respectively. In Tables 4-5 we illustrate these determinations, i.e., where we display $\bar{n}_{I}^{D}$ (measured) for $I=\left(\mathrm{Cl}, \mathrm{Ni}^{+2}\right)$ in the second columns, in comparison with the actual $\bar{n}_{I}^{D}\left(r_{s}\right)$ values in the first columns, and either the corresponding CIP corrected values in the third columns or the $\bar{n}_{C l}^{D}(h w, n N i)$. This comparison clearly highlights the equivalence and self-consistency of the proposed NDIS-based approaches and emphasizes the fact that the validity of Eqns. (11)-(12) and (19) are obviously independent of the presence of ion pairing, i.e., for $\bar{n}_{N i}^{C l}\left(r_{s}\right)=0$, Eqns. (10)-(12) and (19) become identically the same as Eqn. (8). 


\section{Case Study 3: Ion hydration in aqueous $\mathrm{LiCl}$ solutions}

Aqueous lithium chloride solutions have been the target of multiple NDIS over the entire range of solubility ${ }^{1,70,127-136}$, XRD ${ }^{48,137-142}$, X-ray Compton scattering ${ }^{143-144}$, infrared spectroscopy (IR) ${ }^{145}$, dielectric relaxation spectroscopy (DRS) ${ }^{146-150}$, Raman spectroscopy ${ }^{151-152}$, NMR ${ }^{153}$, as well as ab initio and classical molecular simulation studies $59,154-166$. Yet, no clear picture of its hydration behavior emerges from those studies, a situation once again linked to the $\mathrm{Li} \cdots \mathrm{Cl}$ pair association and its effect on the interpretation of ion coordination. For instance, reverse Monte Carlo (RMC) analysis of early data from Narten et al. x-ray and neutron diffraction experiments ${ }^{139}$ by Harsanyi and Pusztai ${ }^{167}$ detected no $\mathrm{Li} \cdots \mathrm{Cl}$ formation even at the highest concentration of $18.5 \mathrm{~m}$. However, the same approach applied to newer diffraction data provided clear evidence for the presence of contact ion pairs characterized as $\bar{n}_{L i}^{C l}(C I P) \quad 0.2$ and $\bar{n}_{L i}^{C l}(C I P) \quad 1.0$ for the $3.0 m$ and $14.0 m$ solution, respectively ${ }^{142}$. The newest RMC outcome becomes consistent with the NDIS studies by Winkel et al. ${ }^{135}$ on a $1.38 \mathrm{~m}$ system at ambient conditions that exhibited $0.2 \bar{n}_{L i}^{C l}(C I P) \quad 0.3$, and by Ichikawa et al. ${ }^{129}$ on a $12.8 m$ solution at higher temperature as illustrated in Figure 10. Additional support for the existence of contact $\mathrm{Li} \cdots \mathrm{Cl}$ pair formation comes from diverse experimental techniques including NMR and differential thermal analysis (DTA) ${ }^{153}$, DRS ${ }^{148}$, IR ${ }^{145}$, x-ray Compton scattering ${ }^{144}$, as well as ab initio molecular dynamics ${ }^{163}$. However, neither DRS ${ }^{149}$ nor Raman spectroscopy ${ }^{168}$ is able to detect contact $\mathrm{Li} \cdots \mathrm{Cl}$ pairs for concentration below $1 \mathrm{~m}$.

While the spectroscopic evidence for contact $\mathrm{Li} \cdots \mathrm{Cl}$ pair configurations is strong, the diffraction counterpart is not so, and the source of controversy usually hinges around the identification of the structural signature for the CIP configuration as we have already discussed it in the previous case studies. To illustrate the point according to proposed developments, and link the literature findings with our simulation results, here we revisit the analysis of the hydration behavior of aqueous $\mathrm{LiCl}$ solutions over a wide range of concentration, i.e., $3 m$ 20. As a representative example, in Figure 11 we display the first order differences $G_{\mathrm{Cl}}^{\text {solv norm }}(r)$ for the ${ }^{n a t} \mathrm{Cl} /{ }^{37} \mathrm{Cl}$ substitution in heavyand null-aqueous environments, where we clearly identify and isolate the contact $\mathrm{Li} \cdots \mathrm{Cl}$ 
pair ( CIP) configuration. Consequently, we are able to assess its contribution to the corresponding "measured" coordination numbers, Eqns. (11) and (13), and correct them according to the proposed methodology as illustrated in Figure 12 as a function of salt molality, as well as in Tables 6-7.

Table 6: First coordination numbers of $\mathrm{Cl}$ in $14 \mathrm{~m} \mathrm{LiCl}$ aqueous solutions from integrals over $G_{C l}^{\text {solv,norm }}(r)$ and $G_{C l, n L i}^{h w, n o r m}(r)$

\begin{tabular}{|c|c|c|c|}
\hline \hline $\bar{n}_{C l}^{D}\left(\right.$ Eqn. 8), $r_{s}$ & $\bar{n}_{C l}^{D}\left(\right.$ Eqn. 10), $r_{L}, r_{U}$ & $\bar{n}_{C l}^{D}(\text { Eqns. 11-12) })^{(a)}$ & $\bar{n}_{C l}^{D}\left(\right.$ Eqn. 21), $r_{L}, r_{U}$ \\
\hline \hline $4.39,2.96 \AA$ & $3.86,0.0 \AA, 2.88 \AA$ & 4.28 & $4.3,0.0 \AA, 2.96 \AA$ \\
\hline
\end{tabular}

Table 7: First coordination numbers of $\mathrm{Li}^{+}$in $14 \mathrm{~m} \mathrm{LiCl}$ aqueous solutions from integrals over $G_{L i}^{\text {solv,norm }}(r)$

\begin{tabular}{|c||c|c|}
\hline $\bar{n}_{L i}^{D}\left(\right.$ Eqn. 8), $r_{s}$ & $n_{L i}^{D}\left(\right.$ Eqn. 13), $r_{L}, r_{U}$ & $\bar{n}_{L i}^{D}($ Eqns. 13-14) \\
\hline \hline $6.0,3.05 \AA$ & $8.1,2.23 \AA, 3.03 \AA$ & 6.0 \\
\hline
\end{tabular}

The take home message from Figure 12 and Tables 6-7 is that while $\bar{n}_{L i}^{O}\left(r_{s}\right)$ can be determined from the integration of either the heavy water or the null water neutronweighted radial distribution function without the interference of the potential occurrence of ion pairing, this is not the case for $\bar{n}_{L i}^{D}\left(r_{s}\right)$, as a consequence of the $C I P$ contributions proportional to $\bar{n}_{L i}^{C l}\left(r_{s}\right)$. The top of Figure 12 also highlights the fact that $\bar{n}_{L i}^{O}\left(r_{s}\right)$ exhibits a significant concentration dependence at ambient conditions, with a similar trend for $\bar{n}_{L i}^{D}\left(r_{s}\right)$ after the $C I P$ correction. In contrast $\bar{n}_{L i}^{D}$ (measured), extracted from the heavywater neutron-weighted distribution function, appears almost constant within the entire range of salt concentration. This behavior prompts us to note that if we did not sort the coordinating atoms around $\mathrm{Li}^{+}$according to their identities, i.e., by just counting its nearest interactions sites, $\mathrm{Li}^{+}$would exhibit an 'apparent' first coordination number $\bar{n}_{L i}^{(C l \oplus O)}$ (apparent) 4 while $\bar{n}_{L i}^{D}$ (measured) 8 and thus, satisfying the 2:1 (H/O)-ratio 
for water that is oftentimes loosely used as an indicator of consistency in the extracted coordination results.

Given the challenging nature of the detection of CIP configurations, it would be convenient either to avoid altogether the need for its assessment, or to have an alternative approach to test the accuracy and consistency of the NDIS-based ion-water coordination numbers. This scenario makes the aqueous $\mathrm{LiCl}$ solutions somewhat special in two important ways: (a) both ions in the salt comprise appropriate contrast for neutron scattering experiments, and (b) as for the cases of ${ }^{n a t} \mathrm{Ni} /{ }^{62} \mathrm{Ni}$ and $\mathrm{H} / \mathrm{D}$ substitution, ${ }^{n a t} \mathrm{Li} /{ }^{6} \mathrm{Li}$ also exhibits a change in the sign of the coherent scattering length so that we can also take advantage of the "null-lithium" environment by choosing the isotopic composition $c_{{ }^{L i}} / c_{\text {nat } L i}=\left(b_{\text {nat }_{L i}} / b_{6_{L i}}\right) \quad 0.95$. This behavior, in principle, allows proposing a "null- lithium" approach ${ }^{53}$ that will provide a direct route to $\bar{n}_{C l}^{D}\left(r_{s}\right)$ from heavy-water NDIS experiments, and consequently, will avoid the need for the CIP correction, Eqn. (11) for heavy water environments, whose manifestation at $r_{s} \simeq 2.43 \AA$ is illustrated in Figure 11.

As per the null-nickel case study, the relevant distribution function $G_{C l}^{h w, n o r m}(r)$ in the null-lithium environment reduces to the simpler form,

$$
G_{C l, n L i}^{h w, n o r m}(r)=\left[A_{C l} g_{O C l}(r)+B_{C l} g_{D C l}(r)+E_{C l} g_{C l C l}(r)\right] /{ }_{C l, n L i}^{h w}
$$

with ${ }_{C l, n L i}^{h w}=A_{C l}+B_{C l}+E_{C l}$ whose behavior for a $14 m \mathrm{LiCl}$ is illustrated in Figure 13. Consequently, the coordination number $\bar{n}_{C l}^{D}\left(r_{s}\right)$ follows from the counterpart of Eqn. (9) for a null-cation environment, i.e.,

$$
\bar{n}_{C l}^{D}\left(r_{s}\right)=\left(\begin{array}{lll}
4 & { }_{D} & { }^{h w} \\
C l, n L i
\end{array} / B_{C l}\right) \int_{0}^{r_{s}} G_{C l, n L i}^{h w, n o r m}(r) r^{2} d r
$$

as given in Table 6 in comparison with the corresponding values obtained from the corrected heavy-water estimations.

c) Illustration of the methodology for model systems involving hetero-atomic anions 
Up to this point we have studied simple salts comprising monoatomic ions that have been the most frequently targeted by scattering experiments. However, there is another type of simple salts comprising hetero-atomic anions, such as oxyanions, resulting in 'complexities' that are more challenging to interpret experimentally. On the one hand, the major contribution to the scattering originates in the stronger water-cation interactions that overwhelm the rather weaker oxyanion-water interactions. On the other hand, the fact that the anion's oxygen-water and water's oxygen-water interactions are comparable, i.e., exhibiting correlation peaks at similar distances, makes it difficult to discriminate anion's oxygen-water from water's oxygen-water interactions, consequently, to provide adequate information about the anion coordination.

Here we will take advantage of some very recent developments on ${ }^{n a t} O_{N} /{ }^{18} O_{N}$ substitution ${ }^{169-170}$ that open to the possibility of sequential substitution of two different anion sites. This feature increases our chances for a fuller characterization of the hydration structure of oxyanions, and the potential occurrence of oxyanion-cation pairs as we will discuss below. While oxyanion hydration has been traditionally studied by $\mathrm{x}$-ray and neutron diffraction, as well as ultra-violet/visible (UV/vis), NMR, and Raman spectroscopies, the spectroscopic tools might not be reliable enough for the study of ion pair association in aqueous electrolytes involving non-contact ion pair formation, i.e., SShIP and solvent-separated ( SSIP) configurations ${ }^{171}$.

Note that for the microstructure of a simple aqueous $M^{+}\left[X O_{n}\right]$ solution (with $X=N, S, C l, P)$ involving $s$ isotopic scattering species, there are $s(s+1) / 2$ independent radial distribution functions contributing to the neutron weighted distribution function, Eqn. (4), i.e., between 10 and 15 for these oxyanions. For NDIS experiments involving $X / X$ and $M / M$ substitutions we will deal with 10 correlation functions, though if we also introduced the isotopic substitution ${ }^{n a t} O_{X} /{ }^{18} O_{X}$ on the anion only (i.e., not on the water), then we will have five additional correlation functions. Thus, the original Eqn. (7) for the first-order difference must be supplemented as follows, 


$$
\begin{aligned}
G_{X}^{\text {solv, norm }}(r)=[ & A_{X} g_{O_{W} X}(r)+B_{X} g_{D X}(r)+C_{X} g_{H X}(r)+ \\
& \left.D_{X} g_{M X}(r)+E_{X} g_{X X}(r)+F_{X} g_{O_{X} X}(r)\right] / /_{X}^{\text {solv }}
\end{aligned}
$$

and,

$$
\begin{aligned}
G_{O}^{\text {solv, norm }}(r)=[ & A_{O} g_{O_{W} O_{X}}(r)+B_{O} g_{D O_{X}}(r)+C_{O} g_{H O_{X}}(r)+ \\
& \left.D_{O} g_{M O_{X}}(r)+E_{O} g_{O_{X} O_{X}}(r)+F_{O} g_{X O_{X}}(r)\right] /_{O}^{\text {solv }}
\end{aligned}
$$

with $\quad{ }_{\ell}^{\text {solv }}=A_{\ell}+B_{\ell}+C_{\ell}+D_{\ell}+E_{\ell}+F_{\ell}=G_{\ell}^{\text {solv }}(0)$.

In what follows we will invoke these expressions for the interpretation of the coordination behavior of oxyanions. Note that, in principle $g_{X O_{X}}=g_{O_{X} X}$ will contain intra-molecular correlations centered at the corresponding bond-length distance $\ell_{X} O_{X}$, i.e., $\sim 1.27 \AA$ for $\mathrm{NO}_{3}$. Consequently, the experimental $G_{O}^{\text {solv,norm }}(r)$ and $G_{X}^{\text {solv,norm }}(r)$ will usually contain those intra-molecular correlations, while (by construction) the simulation counterparts will not. As discussed elsewhere ${ }^{63}$, the intra-molecular contributions to the experimental $G_{O}^{\text {solv,norm }}(r)$ and $G_{X}^{\text {solv,norm }}(r)$ can be used to test the internal consistency of the NDIS rawdata.

\section{Case Study 4: Ion hydration in aqueous $\mathrm{LiNO}_{3}$ solutions}

As an oxyanion, $\mathrm{NO}_{3}$ has been characterized as exhibiting mild interactions with water, i.e., weakly hydrated as a result of its small charge and relatively large effective ionic that induce limited distortion of the hydrogen bonding of the surrounding water ${ }^{172-}$ 173. These features might have contributed to the large disparity in the reported water coordination of the nitrate anion from different experimental sources, including NMR ${ }^{174-}$ ${ }^{180}$, IR ${ }^{181-187}$, Raman ${ }^{173-174,188-197}$, XRD ${ }^{172-173,190,193,195-204}$, as well as NDIS ${ }^{205-210}$, and point to unresolved concerns regarding the determination and interpretation of the hydration microstructure of nitrates in a variety of aqueous environments ${ }^{63}$.

Some of those concerns point to the potential for ion-pair formation, depending on the cation's identity and the salt concentration. In fact, there is significant 
experimental evidence for the occurrence of either contact or water-shared ion pairing in aqueous nitrate solutions involving a variety of cations ${ }^{180,182,192,204,211-215}$. In particular, the Raman spectroscopic studies of Irish et al. ${ }^{211-212}$ provided strong evidence on the occurrence of $\mathrm{CIP}$ in $\mathrm{NaNO}_{3}$ aqueous solutions for $m$ 2.5, and the presence of SShIP in $\mathrm{LiNO}_{3}$ aqueous solutions with $m>4.6$. Moreover, the combined Raman spectroscopic and XRD analysis of Smirnov et al. ${ }^{202}$ on $\mathrm{Ca}\left(\mathrm{NO}_{3}\right)_{2}$ aqueous solutions indicated that $~ 55 \%$ and $37 \%$ of the $\mathrm{Ca}^{+2}$ in $8.9 \mathrm{~m}$ and $4.5 \mathrm{~m}$ solutions, respectively, are directed bonded to the $\mathrm{NO}_{3}$, i.e., participating in $\mathrm{CIP}$ configurations.

Therefore, in addition to detecting the presence of ion pair formation, we must isolate their contribution for an adequate assessment of the coordinating nitrate environment, and identify the overlapping of several inter- and intra-atomic correlations within the radial distance, $(2.0<r(\AA)<4.4)$, where we expect the occurrence of the $\mathrm{NO}_{3} \mathrm{H}_{2} \mathrm{O}$ correlations. Unless we provide ways to identify and deconvolute these contributions, they will obstruct both the XRD determination of the water orientation around the nitrate ion caused by the weak hydrogen scattering, and the interpretation of the coordinating environment from heavy-water NDIS involving ${ }^{14} \mathrm{~N} /{ }^{15} \mathrm{~N}$ substitution.

We have recently confronted some of those issues by proposing a novel approach for the accurate determination of the coordination behavior of the nitrate ion, based on the interplay between NDIS involving the double isotopic substitution on the nitrate group, i.e., ${ }^{14} \mathrm{~N} /{ }^{15} \mathrm{~N}$ and ${ }^{\text {nat }} O_{N} /{ }^{18} O_{N}$ in heavy-water and null-water environments ${ }^{63}$. The proposed approach hinges around two innovations: (i) the use of the nearly six-times larger scattering-length contrast between ${ }^{18} \mathrm{O}$ and ${ }^{n a t} \mathrm{O}-0.204 \pm 0.003 \mathrm{fm}{ }^{169}-$ than the earlier tabulated value ${ }^{51}$, i.e., making feasible NDIS experiments using ${ }^{n a t} \mathrm{O} /{ }^{18} \mathrm{O}$ substitution ${ }^{170}$ specially considering that 3-times larger concentration of scattering $O$ sites than $N$ sites, and (ii) the alternating use of heavy- and null-water as a means to extract coordination information for both the water-hydrogen (not achievable by XRD due to the extremely weak hydrogen scattering) and the water-oxygen around the nitrate sites which has been only possible from XRD experiments. 
In what follows we analyze the behavior of the neutron-weighted pair correlation functions for the double ${ }^{14} \mathrm{~N} /{ }^{15} \mathrm{~N}$ and ${ }^{n a t} \mathrm{O}_{N} /{ }^{18} \mathrm{O}_{N}$ substitutions in nitrate ion in heavyand null-aqueous solutions, according to the simulated microstructure of $6.16 \mathrm{~m} \mathrm{LiNO}_{3}$ and the coherent scattering lengths from the available literature as collected in Table 8 .

To facilitate the interpretation of the corresponding first-order difference $G_{N}^{\text {solv,norm }}(r)$ and $G_{O}^{\text {solv,norm }}(r)$ for both types of solvents, we plot the individual contributions from each pair interactions in Figures 14-15, and collect the coefficients of the two sets of first-order differences in Tables 9-10 to highlight the contrasting magnitudes of these coefficients not only for the pair interactions, i.e., $A_{\ell}, B_{\ell} \gg\left|D_{\ell}\right|, E_{\ell}, F_{\ell}$, but also the type of isotopic substitution, i.e., $A_{N}, B_{N} \gg A_{O}, B_{O}$. These inequalities highlight the facts that (a) the two most relevant contributions to $G_{\ell}^{\text {solv,norm }}(r)$ come from the pair interactions between the $\ell$ substituted species and the surrounding water, and (b) the change of coherent scattering length for the ${ }^{n a t} O_{N} /{ }^{18} O_{N}$ substitution is significantly smaller than that for the ${ }^{14} \mathrm{~N} /{ }^{15} \mathrm{~N}$ substitution.

Table 8: Coherent neutron scattering lengths for aqueous $\mathrm{LiNO}_{3}$

\begin{tabular}{|c|c|c|}
\hline Species & $b_{\text {coh }}(f m)$ & Ref. \\
\hline \hline${ }^{14} N$ & 9.37 & 51 \\
\hline${ }^{15} N$ & 6.44 & 51 \\
\hline${ }^{n a t} N$ & 9.36 & 51 \\
\hline${ }^{2} \mathrm{H}$ & 6.67 & 51 \\
\hline${ }^{n a t} \mathrm{O}$ & 5.805 & 51 \\
\hline${ }^{18} \mathrm{O}$ & 6.009 & 169 \\
\hline${ }^{n a t} \mathrm{Li}$ & 1.9 & 51 \\
\hline${ }^{6} \mathrm{Li}$ & 2.0 & 51 \\
\hline${ }^{7} \mathrm{Li}$ & 2.22 & 51 \\
\hline
\end{tabular}


As we have previously found for the case of $3.5 m \mathrm{KNO}_{3}{ }^{63}, G_{N}^{h w, \text { norm }}(r)$ exhibits two prominent peaks, in this case at slightly larger distances, i.e., at $\sim 2.77 \AA$ and $\sim 3.87 \AA$ corresponding to the nearest $D \cdots N$ interactions, and the combination of contributions from the second nearest $D \quad N$, the $O_{w} \quad N$, and the negative contribution from the contact $L i \cdots N$ pair interactions. Moreover, the overlapping of $O_{w} N$, $L i \cdots N$, and $D N$ correlation peaks within $3.2 r(\AA) 4.7$, precludes the determination of the second $D \cdots N$ coordination by integration of the second peak of $G_{N}^{h w, n o r m}(r)$

Table 9: Coefficients of the first-order difference of weighted distributions for the ${ }^{14} \mathrm{~N} /{ }^{15} \mathrm{~N}$ substitution in $6.16 \mathrm{~m} \mathrm{LiNO}_{3}$ heavy- and null-aqueous solutions

\begin{tabular}{|c|c|c|}
\hline Coefficient $^{(a)}$ & heavy water & null water \\
\hline$A_{N}$ & 0.2620122 & 0.2620122 \\
\hline$B_{N}$ & 0.6021091 & 0.2163830 \\
\hline$C_{N}$ & 0.0 & 0.2163830 \\
\hline$D_{N}$ & $7.914160 \mathrm{E}-03$ & $7.914160 \mathrm{E}-03$ \\
\hline$E_{N}$ & $3.292707 \mathrm{E}-02$ & $3.292707 \mathrm{E}-02$ \\
\hline$F_{N}$ & $7.253952 \mathrm{E}-02$ & $7.253952 \mathrm{E}-02$ \\
\hline solv & 0.9616738 & 0.3595647 \\
\hline$N$
\end{tabular}

(a) In $\mathrm{fm}^{2}$ units

Table 10: Coefficients of the first-order difference of weighted distributions for the ${ }^{n a t} \mathrm{O}_{N} /{ }^{18} \mathrm{O}_{N}$ substitution in $6.16 \mathrm{~m} \mathrm{LiNO}_{3}$ heavy- and null-aqueous solutions

\begin{tabular}{|c|c|c|}
\hline Coefficient $^{(a)}$ & heavy water & null water \\
\hline \hline$A_{O}$ & $5.472748 \mathrm{E}-02$ & $5.472748 \mathrm{E}-02$ \\
\hline$B_{O}$ & 0.1257648 & $4.519672 \mathrm{E}-02$ \\
\hline$C_{O}$ & 0.0 & $4.519672 \mathrm{E}-02$ \\
\hline$D_{O}$ & $1.653060 \mathrm{E}-03$ & $1.653060 \mathrm{E}-03$ \\
\hline$E_{O}$ & $1.541781 \mathrm{E}-02$ & $1.541781 \mathrm{E}-02$ \\
\hline$F_{O}$ & $8.143496 \mathrm{E}-03$ & $8.143496 \mathrm{E}-03$ \\
\hline solv & 0.2024005 & $7.663573 \mathrm{E}-02$ \\
\hline
\end{tabular}

(a) In $\mathrm{fm}^{2}$ units 
Note also that the first peak of $G_{N}^{h w, n o r m}(r)$ in Figure 14 comprises mostly the contribution from the first peak of the corresponding $g_{D N}(r)$, yet there are two compensating (i.e., one negative and the other positive) contributions from the $O_{w} \quad N$ and $L i \cdots N$. Thus, the conventional calculation of the first coordination number $\bar{n}_{N}^{D}\left(r_{s}\right)$ by integration of $G_{N}^{h w, n o r m}(r)$ as usually done ${ }^{208}$, i.e.,

$$
\bar{n}_{N}^{D}(r)=\left(\begin{array}{lll}
4 & & h w \\
& D & N
\end{array} / B_{N}^{h w}\right) \int_{r_{L}}^{r_{U}} G_{N}^{h w, n o r m}(r) r^{2} d r
$$

might result in a corrupted coordination number because the upper limit of integration $r_{U} 3.16 \AA$, corresponding to the location of the first minimum of $G_{N}^{h w, n o r m}(r)$, is slightly smaller than the actual value of $r_{s} 3.32 A$ from the $g_{D N}(r)$. In fact, the direct determination of $\bar{n}_{N}^{D}\left(r_{s}\right)$ using Eqn. (8) indicates that the (true) first coordination number $\bar{n}_{N}^{D}\left(r_{s}=3.32 \AA\right) \quad 7.0$ while the estimated value from Eqn. $(25)$ is $\bar{n}_{N}^{D}\left(r_{U}=3.16 \AA\right) \quad 6.3$. Note that we found slightly smaller values for the corresponding coordination numbers for the less concentrated $3.5 \mathrm{~m} \mathrm{KNO}_{3}$ aqueous solution studied previously, i.e., $\bar{n}_{N}^{D}\left(r_{s}=3.32 \AA\right) \quad 5.9$ and $\bar{n}_{N}^{D}\left(r_{U}=3.17 \AA\right) \quad 5.2$, respectively. This comparison highlights a frequently overlooked fact that the equivalence between the true coordination, Eqn. (8), and the NDIS-related coordination, Eqn. (25), relies on the absence of any contribution from any other correlations in the $\left[r_{L}, r_{U}\right]$ radial interval.

For the null aqueous environment there is no contribution from hydrogen interactions to $G_{N}^{n w, n o r m}(r)$, Figure 14 (bottom), whose first peak centered at $r \quad 3.72 \AA$ comprises two main components, i.e., a major contribution from $O_{w} \cdots N$ interactions that overlap with the contact $L i \cdots N$ pair configurations. As for the case of $\bar{n}_{N}^{D}\left(r_{U}\right)$ from $G_{N}^{h w, n o r m}(r)$, Eqn. (25), other smaller contributions from the $N \quad N$ and $O_{N} \quad N$ pair correlation peaks affect the outcome of the conventional determination of the water coordination around the nitrogen site, i.e., 


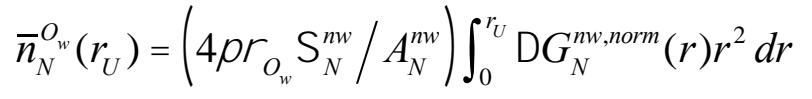

as illustrated in table 11 where we collect the resulting water-oxygen coordination around the nitrogen site of the nitrate ion, $\bar{n}_{N}^{O_{w}}\left(r_{U}\right)$ according to the direct integration of the $g_{O_{w} N}(r)$, integral ${ }^{(c)}$ below, and the NDIS-based estimate based on Eqn. (26).

In Figure 15 we display the corresponding $G_{O}^{h w, n o r m}(r)$ and $G_{O}^{n w, n o r m}(r)$ counterparts to Figure 14. Note that $G_{O}^{h w, n o r m}(r)$ exhibits an almost completely decoupled first peak associated with the $D O_{N}$ correlations centered at $r \quad 1.82 \AA$ resembling the $D O_{W}$ peak for pure water ${ }^{216}$, except for the small negative contribution from the contact $L i \cdots O_{N}$ pair, followed by a wider multi-peak from the overlapping of the second $D \quad O_{N}$ coordination and the two $O_{W} \quad O_{N}$ correlation peaks.

Table 11: First water coordination of the nitrate ion in $6.16 \mathrm{~m} \mathrm{LiNO}_{3}$ heavy- and nullaqueous solutions according to the direct and the NDIS-based expressions

\begin{tabular}{|c|c|c|}
\hline pairinteractions & direct integral $\left(r_{s}\right)^{(\mathrm{c})}$ & $\operatorname{NDIS~integral}\left(r_{U}\right)^{(\mathrm{d})}$ \\
\hline $\begin{array}{ll}O_{W} & N\end{array}$ & $14.7(4.91 \AA)$ & $17.9(4.98 \AA)^{(\mathrm{b})}$ \\
\hline$D \quad N$ & $7.0(3.32 \AA)$ & $6.3(3.16 \AA)^{(a)}$ \\
\hline$O_{W} \quad O_{N}$ & $4.0(3.56 \AA)$ & $3.8(3.48 \AA)^{(\mathrm{b})}$ \\
\hline$D \quad O_{N}$ & $2.4(2.53 \AA)$ & $2.2(2.45 \AA)^{(a)}$ \\
\hline $\mathrm{Li} \quad N$ & $0.5(3.64 \AA)$ & --- \\
\hline $\begin{array}{ll}L i & O_{N}\end{array}$ & $0.19(2.61 \AA)$ & $0.18(2.45 \AA)^{(b)}$ \\
\hline
\end{tabular}

${ }^{(a)}$ heavy-water; ${ }^{(b)}$ null-water; ${ }^{(c)} \bar{n}_{b}^{a}\left(r_{s}\right)=4{ }_{a}^{r_{s}} g_{a b}(r) r^{2} d r$;

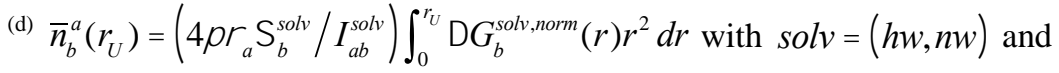
$I_{a b}^{\text {solv }}=\left(A_{b}^{\text {solv }}\right.$ if $a=O_{w} ; B_{b}^{\text {solv }}$ if $\left.a=D\right)$

If we ignore the small $L i \cdots O_{N} C I P$ contribution to the first peak of $G_{O}^{h w, n o r m}(r)$, then its integral will provide an adequate estimate - by comparison against the corresponding integral ${ }^{(\mathrm{c})}$ below - for the first coordination $\bar{n}_{O_{N}}^{D}\left(r_{s}\right)$ as clearly depicted in 
Table 11. This number can then corrected for any $L i \cdots O_{N}$ contribution by taking advantage of the complete deconvolution of this peak in $G_{O}^{n w, n o r m}(r)$, i.e.,

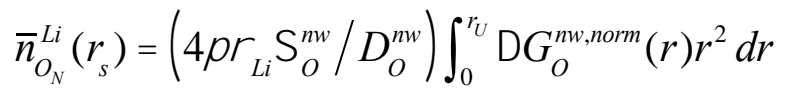

so that,

$$
\begin{aligned}
& \bar{n}_{O_{N}}^{D}(\text { measured })=\left(\begin{array}{lll}
4 & & h w \\
& D & O
\end{array} / B_{O}^{h w}\right) \int_{0}^{r_{U}} G_{O}^{h w, n o r m}(r) r^{2} d r \\
& =\left(\begin{array}{ll}
4{ }_{D} & / B_{O}^{h w}
\end{array}\right) \int_{0}^{r_{U}}\left(B_{O}^{h w} g_{D O_{N}}(r)+D_{O} g_{L i O_{N}}(r)\right) r^{2} d r \\
& =\bar{n}_{O_{N}}^{D}\left(r_{s}\right)+\underbrace{\left(\begin{array}{ll}
4 & { }_{D} D_{O} / B_{O}^{h w}
\end{array}\right) \int_{0}^{r_{U}} g_{L i O_{N}}(r) r^{2} d r}_{\text {CIP contribution }} \\
& =\bar{n}_{O_{N}}^{D}\left(r_{s}\right)+\left(b_{L i} / b_{D}\right) \bar{n}_{O_{N}}^{L i}\left(r_{s}\right)
\end{aligned}
$$

Table 12: First coordination numbers of $\mathrm{NO}_{3}$ in $6.16 \mathrm{~m} \mathrm{LiNO}_{3}$ aqueous solutions from integrals over $G_{O}^{\text {solv,norm }}(r)$ and $G_{N}^{\text {solv,norm }}(r)$

\begin{tabular}{|c|c|c|}
\hline $\bar{n}_{O_{N}}^{D}\left(\right.$ Eqn. 8), $r_{s}$ & $\bar{n}_{O_{N}}^{D}\left(\right.$ Eqn. 28), $r_{U}$ & $\bar{n}_{O_{N}}^{D}($ Eqns. 27-28) \\
\hline \hline $2.4(2.53 \AA)$ & $2.2(2.45 \AA)$ & 2.25 \\
\hline
\end{tabular}

Moreover, note that the second peak of $G_{O}^{n w, n o r m}(r)$ centered at $r \quad 4.75 \AA$, from a combination of the smaller solvent-shared $L i \cdots O_{N}$ pair and $N O_{N}$ contributions, describes the corners of a deformed tetrahedral configuration locating the water-oxygens and the nitrate counterparts. Should this tetrahedral distribution between the nitrateoxygens and water-oxygens exist, as suggested by Caminiti et al. ${ }^{172}$ (see their Figure 3) and Dagnall et al. ${ }^{199}$, we would find each nitrate-oxygen coordinating 3 water-oxygens. According to Table 11, the current molecular models describe a tetra- rather than the tricoordination suggested by these authors, yet, the nature of the cation (and its own hydration behavior) might play an important role on this outcome. In fact, our recent study of the hydration of $\mathrm{NO}_{3}$ in a $3.5 \mathrm{~m} \mathrm{KNO}_{3}$ aqueous solution under the same nitrate 
potential model description resulted in a nitrate-oxygen coordinating 2 water-oxygens ${ }^{63}$. Therefore, according to these results (i.e., Table 5 of Ref. 63 and Table 11 of the present work) the frequently used assumption $\bar{n}_{N}^{O_{w}}\left(r_{s}^{O_{w} N}\right) \quad 3 \bar{n}_{O_{N}}^{O_{w}}\left(r_{s}^{O_{w} O_{N}}\right)$ in the interpretation of XRD data ${ }^{199,217-218}$ should not necessarily be taken for granted, given the fact that the radii of the coordination shells for these two types of interactions are significantly different (i.e., $r_{s}^{O_{w}^{N}} \square r_{s}^{O_{w} O_{N}}$, see Table 11).

The hydration behavior of nitrates has been usually described in terms of the weakness of the water-oxyanion interactions whose immediate assumption is the absence of a second water-nitrate coordination in the modeling of XRD experiments ${ }^{219}$. However, as Kameda et al. ${ }^{208}$ conjectured the location of the second-nearest $D \quad N$ correlation peak centered at $4.1<r(\AA)<4.2$, we found that the $g_{D N}(r)$ exhibits two prominent peaks centered at $r \square 2.77 \AA$ and $r \square 4.19 \AA$, respectively, as clearly illustrated in Figure 14 (similar behavior was observed in the study of $3.5 \mathrm{~m} \mathrm{KNO}_{3}$ solution, see Figure 1 in Ref. 63). Yet, the conventional heavy-water based NDIS experiment will not be able to provide information about the corresponding coordination due to the peak overlapping between $O_{N} \quad N, O_{W} \quad N$, and $D \quad N$. For that purpose we have recently proposed an NDIS route that in principle will provide an estimation of the second coordination number $n_{D}^{N}\left(r_{L}, r_{U}\right)$ as follows (see Appendix A of Ref. 63 for details),

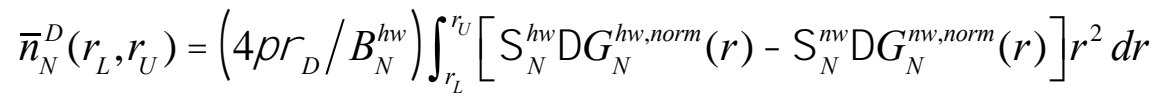

where $G_{N}^{h w, \text { norm }}(r)$ and $G_{N}^{n w, \text { norm }}(r)$ are given in Figure 14, while $r_{L}$ and $r_{U}$ are the location of the minima defining the second peak of the $g_{D N}(r)$.

The resulting second coordination numbers $\bar{n}_{N}^{D}\left(r_{L}, r_{U}\right)$ from Eqn. (29) are compared in Table 13 against those from the direct integration of $g_{D N}(r)$, Eqn. (8), for two alternative $r_{U}$ values to highlight the presence of a shoulder in the relevant integration domain of $G_{N}^{h w, n o r m}(r)$. Note that these coordination numbers are comparable to those found for $3.5 \mathrm{~m} \mathrm{KNO}_{3}$ aqueous solutions. 
Table 13: Second water-hydrogen coordination of the nitrate ion according to the direct and the derived NDIS-based expressions

\begin{tabular}{|c|c||c|}
\hline pair interactions & direct integral $\left(r_{L}, r_{U}\right)^{\text {(a) }}$ & $\operatorname{NDISintegral}\left(r_{L}, r_{U}\right)^{(\text {b) }}$ \\
\hline \hline$D N$ & $22.4(3.32 \AA, 4.90 \AA)$ & $23.3(3.16 \AA, 4.90 \AA)$ \\
\hline$N$ & $34.5(3.32 A, 5.54 \AA)$ & $35.4(3.16 \AA, 5.54 \AA)$ \\
\hline
\end{tabular}

(a) $\bar{n}_{N}^{D}\left(r_{s}\right)=4 \quad{ }_{D}{ }_{r_{L}}^{r_{U}} g_{D N}(r) r^{2} d r$ (Eqn. (8))

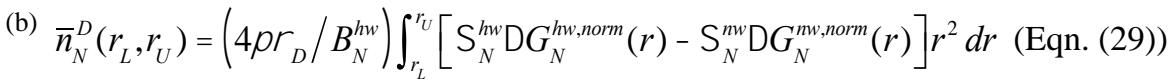

\section{LINK BETWEEN THE DEGREE OF $M^{v+} \cdots X^{v-}$ PAIR FORMATION AND THE $M^{v+} \cdots X^{v-}$ PAIR RADIAL DISTRIBUTION FUNCTION}

The developments in the previous sections have given us the tools to not only detect the presence of CIP between an anion (or an oxyanion site) and the cation, but also to isolate it, and finally estimate a representative coordination number. The unresolved question at this point concerns the extent of such an ion-pair association described as a degree of ion pairing and linked to an association constant, rather than as a coordination number $\bar{n}_{X}^{M}\left(r_{L}, r_{U}\right)$ - and discriminated by the type of configuration as $C I P, S S h I P$, and SSIP according to the Eigen-Tamm classification ${ }^{220}$. Thus, in what follows we invoke a somewhat less-known rigorous formalism to make such a connection 221

The degree of $M^{v+} \cdots X^{\nu-}$ pair association ${ }_{+}$can be expressed in terms of the corresponding $M^{v+} \cdots X^{v-}$ pair distribution function $G_{+}(r)$ (to be derived and not to be confused with the conventional $\left.g_{+}(r)\right)$, as follows,

$$
{ }_{+}\left(d_{+}\right)={ }_{0}^{d_{+}} G_{+}(r) d r
$$

under the condition that, in the thermodynamic limit, $\lim _{d_{+} \rightarrow \infty}{ }_{+}\left(d_{+}\right)=1.0$, where $d+$ typically denotes the largest distance where the $M^{v+} \cdots X^{v-}$ pairs are counted, such as the location of the first (for contact ion pairs) or second valley (for contact plus solvent- 
shared ion pairs) of $g_{+}(r)$. In Eqn. (30) $G_{+}(r) r$ measures the probability of finding the $X$ species in the spherical shell of thickness $r$, separated by a distance $r$ from the $M^{+}$species, when neither $X$ nor $M^{+}$participates in any additional pairing within the radial distance $r$. From a statistical mechanical point of view, $G_{+}(r)$ can then be written as an integral equation,

$$
G_{+}(r)=4 \quad g_{+}(r) r^{2} P(r) P_{+}(r)
$$

where $P(r)\left(P_{+}(r)\right)$ denotes the probability that an $X \quad\left(M^{+}\right)$separated by a distance $r$ from an $M^{+}(X)$ does not engage in an ion pair with any other ion of the opposite charge, i.e.,

$$
\begin{aligned}
& P(r)=1 \quad{ }_{0}^{r} G_{+}(s) d s \\
& P_{+}(r)=1 \quad{ }_{0}^{r} G_{+}(s) d s
\end{aligned}
$$

Therefore, the entire formalism hinges around being able to determine $P(r)$ and $P_{+}(r)$, through the simultaneous solution of Eqns. (31)-(32) under the required conditions of system electroneutrality and species conservation. The explicit form of these conditions depends on the type of ionic formula; consequently, here we will illustrate the simplest case of symmetric salts (for the case of asymmetric ion-pairs please see the original manuscript), i.e., where electroneutrality requires $g_{+}(r)=g_{+}(r)$, so that the species conservation reads,

$$
G_{+}(r)={ }_{+} G_{+}(r)
$$

From Eqns. (32)-(33), it becomes obvious that $P(r)=P_{+}(r)$ because ${ }_{+}=$, and by solving the integral equation it follows that,

$$
P(r)=\left[\begin{array}{ll}
1+4 & +\int_{0}^{r} g_{+}(s) s^{2} d s
\end{array}\right]^{1}
$$

Finally, from Eqn. (31)-(34), 


$$
G_{+}(r)=4+g_{+}(r) r^{2} /\left[1+4+\int_{0}^{r} g_{+}(s) s^{2} d s\right]^{2}
$$

which satisfies the required normalization, i.e.,

$$
\int_{0}^{\infty} G_{+}(r) d r=1 \quad\left[1+4+\int_{0}^{\infty} g_{+}(r) r^{2} d r\right]^{1}=1
$$

Note that the corresponding association constant becomes,

$$
K_{a}=\left(+/+{ }^{2}\right){ }_{0}^{d_{+}} G_{+}(r) d r /\left(\begin{array}{ll}
1 & \left.{ }_{0}^{d_{+}} G_{+}(r) d r\right)^{2}
\end{array}\right.
$$

where + and $\gamma_{\mp}$ denote the ion-pair activity and free ion mean activity coefficients, respectively. As the electrolyte concentration approaches infinite dilution, $\lim _{+\rightarrow 0} 1 \int_{0}^{r} G_{+}(s) d s=4 \int_{0}^{r} g_{+}(s) s^{2} d s$ and consequently Eqn. (37) converges to the most frequently used expression ${ }^{222}$,

$$
\begin{aligned}
K_{a} & =4 \int_{0}^{d_{+}} g_{+}^{\infty}(r) r^{2} d r \\
& =\lim _{+\rightarrow 0}\left[\begin{array}{cc}
+{ }_{+}^{1} /\left(\begin{array}{ll}
1 & +
\end{array}\right)^{2}
\end{array}\right]
\end{aligned}
$$

since, $\lim _{+\rightarrow 0}+=1$. While usually we can derive an expression to determine $G_{+}(r)$ from the corresponding (theoretical or simulated) pair distribution function $g_{+}(r)$ such as Eqn. (35), there could be cases for which there is no analytical solution to the corresponding integral equation $^{223}$. For those cases we are still able to extract $G_{+}(r)$ directly from simulation by accumulating the required histogram according to the principles underlying its definition, Eqn. (31), as illustrated and briefly explained in the Appendix of Ref. 223.

The important message here is twofold. On the one hand, for systems with finite electrolyte concentration we can use the anion-cation radial distribution function $g_{+}(r)$ to assess the extent of the ion-pair association according to Eqn. (30). This equation, in 
conjunction with Eqn. (35) for the case under consideration, tells us that the relevant quantity for the assessment of ion pair association is an integral over $G_{+}(r)$ rather than $g_{+}(r)$, a fact that is usually overlooked. On the other hand, for the system at limiting concentrations, the relevant distribution $g_{+}(r)$ is not experimentally accessible, i.e., it requires an indirect simulation route (via potential of mean force) for its evaluation as discussed elsewhere ${ }^{222}$.

Moreover, while the second scenario provides a way to estimate the ion-pair association constant of electrolytes, something that has been achieved successfully for many relevant systems ${ }^{224-226}$, the first scenario might also be a viable approach to provide an estimate of the corresponding association constant, should we have a way to assess the corresponding activity coefficients, through the experimentally determined ionpair coordination numbers $n^{+}\left(r_{s}\right)$. In fact, after recalling its definition, Eqn. (8), Eqn. (37) reduces to,

$$
K_{a}\left(r_{I P}\right) \equiv \gamma_{-+} n_{-}^{+}\left(r_{I P}\right)\left(1+n_{-}^{+}\left(r_{I P}\right)\right) /\left(\gamma_{\mp}^{2} \rho_{+}\right)
$$

Note that Eqn. (39) defines the association constant for the ion-pairs separated by a distance $r_{I P}$, therefore, it should not be confused with the full association constant involving all ion pair configurations, i.e., $C I P+S S h I P+S S I P^{222}$.

In Figures 16-19 we display the radial anion-cation radial distribution functions $g_{+}(r)$, as well as their corresponding ion-pair radial distribution functions $G_{+}(r)$ and degree of ion-pair association ${ }_{+}(r)$. Among other features, note the significant differences in magnitude between $G_{+}(r)$ and $g_{+}(r)$ functions, as well as the correspondence between their two main peaks associated with the CIP and SShIP configurations. All four systems exhibit significant $M^{v+} \cdots X^{v-}$ pair association characterized by $(C I P) \quad 0.76,0.77,0.6$ and 0.36 with $(C I P+S S h I P) \quad 0.8$ for the $\mathrm{CaCl}_{2}, \mathrm{NiCl}_{2}, \mathrm{LiCl}$, and $\mathrm{LiNO}_{3}$, respectively.

Based on the discussed ion-pair formalism we can also make a direct comparison between the simulated degree of ion-pair association and the corresponding values extracted from experimentally measured stability constants, more appropriately, their 
mass action 'concentration products' $\left.K_{a} \quad K_{a}\left(\begin{array}{ll}2 \\ \square\end{array}{ }_{+}\right)={ }_{+}{ }_{+}^{1 /(} / l_{+}\right)^{2}$. For that purpose we invoke data from the tabulation of Rard and Clegg ${ }^{77}$ for the aqueous $\mathrm{CaCl}_{2}$ systems, and plot the resulting $\mathrm{CaCl}^{+}(C I P)$ as a function of the system ionic strength $I(m)$ in comparison with values from molecular simulation in Figure 20. The most obvious feature of this comparison is given by the underestimation of $\mathrm{CaCl}^{+}(\mathrm{CIP})$ by the aqueous models, an outcome that fuels the need for more accurate determinations of association constants 74,77 , novel tools to interpret diffraction and spectroscopic experiments, as well as unambiguous modeling approaches.

It is also instructive for the understanding of the nitrate coordination, and its ionpairing ability, to analyze the microstructural behavior of cation-nitrate interactions in terms of the $L i \cdots N$ spatial distribution function, $g_{L i N}(r, \quad)^{227}$, where the polar angle is defined between the principal $z$ axis (direction perpendicular to the plane defining the nitrate geometry) and the vector position $\boldsymbol{r}$ (relative to the molecule at the origin of the principal axes) of the neighboring $\mathrm{Li}^{+}$, while the azimuthal angle describes the angle formed between the principal $y$ axis and the projection of $\boldsymbol{r}$ onto the $z=0$ plane as depicted in Figure 21. For that purpose we invoke the methodology described in our previous work ${ }^{63}$ where we analyzed the spatial distribution of water around the nitrate ion, though here we are only interested in the spatial distribution of $\mathrm{Li}^{+}$around the anion.

Note that the radial distribution function involved in the definition of the ion-pair distribution function, Eqn. (35), is simply an orientational averaged $g_{\text {LiN }}(r)\left\langle g_{\text {LiN }}(r,),\right\rangle$, counterpart as illustrated at the top of Figure 19, i.e., it describes the mean interaction between the nitrate's nitrogen core and the lithium ion represented by at least three nearest-neighbor shells whose peaks are located at $r \square 3.12 \AA, r \square 5.1 \AA$, and $r \square 7.6 \AA$, respectively. In contrast, the actual spatially distributed $L i \cdots N$ pair interactions exhibit a preference for specific orientations that translate into strong correlations several times larger than the corresponding angleaveraged quantities. This behavior becomes explicit by displaying spatial distribution function $g_{\text {LiN }}(r,)_{=c o n s t}$ at the representative azimuthal angles $=0^{\circ}$ and $=60^{\circ}$ as illustrated in Figure 21a. These azimuthal angles can be taken as reference orientations 
since $=0^{\circ}$ represents the distributions in the plane perpendicular to the nitrate's rigid geometry containing the $N \quad O_{1}$ bond (this distribution is identical to that defined by $=120^{\circ}$ due to molecular symmetry), while $=60^{\circ}$ defines the distribution on the plane bisecting the $\square O_{1} \quad N \quad O_{2}$ angle and containing the $N \quad O_{3}$ bond, i.e., it describes a plane of symmetry.

In Figures $21 \mathrm{~b}-\mathrm{c}$ we illustrate how the main correlation peak in $g_{L i N}(r, \theta)_{\phi=0^{\circ}}$ evolves as we increase the azimuthal angle from $=0^{\circ}$ to $=60^{\circ}$, where the most significant changes occur at the base of the CIP peak and centered at $r \square 3.12 \AA$. The strong single peak $g_{\text {LiN }}\left(r>2.1 \AA, \theta \sim 120^{\circ}\right)_{\phi=0^{\circ}}$ centered at $\theta \sim 120^{\circ}$, transitions to a double peak centered at $\theta \sim 60^{\circ}$ and $\theta \sim 120^{\circ}$, while decreasing its strength as $\rightarrow 60^{\circ}$. Note however that these peaks reach their maximum strength at $r \sim 2.45 \AA$ and $r \sim 3.0 \AA$, i.e., at distances smaller than the actual location of the CIP peak. Similarly, while the radial location of the SShIP peak is about $r \square 5.1 \AA$ with $g_{L i N}(r \sim 5.1 \AA) \sim 2$, the corresponding spatial distribution counterparts $g_{L i N}\left(r \sim 5.1 \AA, 80^{\circ}<\theta<100^{\circ}\right)_{\phi=0^{\circ}} \sim 1 \quad$ and $g_{\text {LiN }}\left(r \sim 5.1 \AA, \theta \sim 90^{\circ}\right)_{\phi=60^{\circ}} \sim 3$. These features attest to the highly inhomogeneous nature of the aqueous environment around the nitrate as illustrated previously ${ }^{63}$.

\section{FINAL REMARKS}

We have provided a detailed account of the fundamentals underlying the microstructural analysis of aqueous electrolytes based on the NDIS approach toward the experimental determination of ion coordination numbers in systems involving both halides anions and oxyanions. In particular, we focused on the potential occurrence of ion-pair formation - a significant hydration phenomenon that is typically overlooked or assumed negligible — identified its microstructural signature in the neutron-weighted distribution functions, suggested new techniques for the direct estimation of the ionpairing magnitude and the consequent correction of its effects on the experimentally measured coordination numbers, and proposed novel approaches to determine anion 
coordination numbers free of ion-pairing contributions through the implementation of null-cation environments.

We illustrated these new developments aimed at the interpretation of four NDIS study cases via molecular simulation, as convenient dry runs for the actual scattering experiments, involving representative electrolyte solutions at ambient conditions comprising aqueous metal halides and nitrates. We should emphasize the challenging nature of the diffraction experiments, especially those involving null environments, and for that reason, we should highlight once more the power of molecular simulation tools that allows taking an atomistic "peek" at the local environment around the isotopicallylabeled species before any experiment is attempted.

The formidable value of that interplay stems from the ability of molecular simulation to provide all pair distribution functions that describe the system microstructure, that allows us to "reconstruct" the NDIS output from the relevant distribution functions, and consequently, to test the accuracy of the "measured" NDISbased coordination numbers against the "direct" counting (true values) from the simulated microstructure. In this context, the novel tools we have recently introduced offer versatile ways (a) to identify the individual partial contributions to the total neutron weighted distribution function (peak size and radial location), (b) to isolate the contributions of ion pair configurations, (c) to "dig up" the hydration behavior of the oxygen-site in oxyanions, and consequently, to assess the actual water coordination around them.

The reader might be rightfully concerned about the realism of the interaction potentials used in the study and how that could affect the outcome of the present analysis. While fixed-charge (i.e., non-polarizable) models have been rather successful in describing the behavior of water and aqueous electrolytes in bulk and interfaces systems, and the models used in this work have shown adequacy to describe the systems of interest, we must also recognize that non-polarizable models are obviously not able to capture some subtleties underlying the inhomogeneneous nature of the polarization phenomena in aqueous environments as attested by our ongoing effort in this matter 228 ${ }^{230}$. Yet, we should also note that, because the molecular simulation in this study provides all the radial distribution functions that fully characterize the system microstructure, we 
can also "reconstruct" the NDIS output from the relevant distribution functions; consequently, we can test the accuracy of the "measured" NDIS-based coordination numbers (e.g., Eqns. (12) and (14)) against the "direct" counting (i.e., Eqn. (8))) from the simulated microstructure. In this sense, the validity of our NDIS-simulation interplay analysis becomes independent of the force field inaccuracies.

In summary, we have offered our assessment on the current state of affairs regarding the understanding of hydration behavior in aqueous electrolytes, and discussed ways to pursue a fruitful interplay between fundamentals, molecular simulation, and experiment. In our view the main challenge now is to confront the difficulties associated with these NDIS experiments and translate the proposed novel schemes into versatile tools for the accurate characterization of ion hydration, a task that is currently being pursued by our research group.

\section{ACKNOWLEDGEMENTS}

This work was supported by the U.S. Department of Energy, Office of Science, Office of Basic Energy Sciences, Chemical Sciences, Geosciences, and Biosciences Division. 


\section{REFERENCES}

1. Enderby, J. E.; Neilson, G. W., The Structure of Electrolyte Solutions. Reports on Progress in Physics 1981, 44, 593-653.

2. Debye, P.; Hückel, E., Theory of Electrolytes. II. The Limiting Law of Electrical Conductivity. Physik 1923, 24, 305-325.

3. Ben-Naim, A., Standard Thermodynamics of Transfer - Uses and Misuses. Journal of Physical Chemistry 1978, 82, 792-803.

4. Ben-Naim, A., Solvation Thermodynamics; Plenum Press: New York, 1987.

5. Rodger, P. M., Local Structure and Local Symmetry in Dilute Solutions. Molecular Physics 1996, 89, 1157-1172.

6. Chialvo, A. A.; Cummings, P. T.; Simonson, J. M.; Mesmer, R. E., Solvation in High-Temperature Electrolyte Solutions. II. Some Formal Results. Journal of Chemical Physics 1999, 110, 1075-1086.

7. Chialvo, A. A., Solvation Phenomena in Dilute Solutions: Formal, Experimental Evidence, and Modeling Implications. In Fluctuation Theory of Solutions: Applications in Chemistry, Chemical Engineering and Biophysics, 1st edition ed.; Matteoli, E.; O'Connell, J. P.; Smith, P. E., Eds. CRC Press: Boca Raton, 2013; pp 191-224.

8. Robinson, R. A.; Stokes, R. H., Electrolyte Solutions, 2nd. (revised) ed.; Butterworths: London, 1970.

9. Barthel, J. M. G.; Krienke, H.; Kunz, W., Physical Chemistry of Electrolyte Solutions; Springer: Darmstadt, 1998; Vol. 5.

10. Desnoyers, J. E.; Verrall, R. E.; Conway, B. E., Electrostriction in Aqueous Solutions of Electrolytes. Journal of Chemical Physics 1965, 43, 243-250.

11. Danielewicz-Ferchmin, I.; Ferchmin, A. R., Hydration of Ions at Various

Temperatures: The Role of Electrostriction. Journal of Chemical Physics 1998, 109, 2394-2402.

12. Davies, C. W., Ion Association; Butterworths: Washington, 1962.

13. Fawcett, W. R., Liquids, Solutions, and Interfaces; Oxford University Press: New York, 2004.

14. Bockris, J. O. M.; Reddy, A. K. N., Modern Electrochemistry; Plenum Press: New York, 1998; Vol. 1.

15. Lee, L. L., A Molecular Theory of Setchenov's Salting-out Principle and Applications in Mixed-Solvent Electrolyte Solutions. Fluid Phase Equilibria 1997, 131, 67-82.

16. Ben-Naim, A., Hydrophobic-Hydrophilic Forces in Protein Folding. In Structure and Reactivity in Aqueous Solutions, Cramer, C. J.; Thrular, D. G., Eds. American Chemical Society: Washington, D.C., 1994; Vol. 568, pp 371-380.

17. Kinoshita, M.; Okamoto, Y.; Hirata, F., Solvation Structure and Stability of Peptides in Aqueous Solutions Analyzed by the Reference Interaction Site Model Theory. Journal of Chemical Physics 1997, 107, 1586-1599.

18. Kirkwood, J. G.; Buff, F. P., The Statistical Mechanical Theory of Solution. I. Journal of Chemical Physics 1951, 19, 774-777. 
19. Matteoli, E.; Mansoori, G. A., Fluctuation Theory of Mixtures. Taylor and Francis: New York, 1990; Vol. 2.

20. Smith, P. E.; Matteoli, E.; O' Connell, J. P., Fluctuation Theory of Solutions: Applications in Chemistry, Chemical Engineering and Biophysics; CRC Press: Boca Raton, 2013.

21. McMillan, W. G.; Mayer, J. E., The Statistical Thermodynamics of Multicomponent Systems. The Journal of Chemical Physics 1945, 13, 276-305.

22. Vafaei, S.; Tomberli, B.; Gray, C. G., Mcmillan-Mayer Theory of Solutions Revisited: Simplifications and Extensions. The Journal of Chemical Physics 2014, $141,-$.

23. Cabezas, H.; Oconnell, J. P., Some Uses and Misuses of Thermodynamic Models for Dilute Liquid Solutions. Industrial \& Engineering Chemistry Research 1993, 32, 2892-2904.

24. Lee, L. L., Thermodynamic Consistency and Reference Scale Conversion in Multisolvent Electrolyte Solutions. Journal of Molecular Liquids 2000, 87, 129147.

25. Mazo, R. M., Prolegomenon to the Fluctuation Theory of Solutions. In Fluctuation Theory of Solutions: Applications in Chemistry, Chemical Engineering and Biophysics, Matteoli, E.; O'Connell, J. P.; Smith, P. E., Eds. CRC Press: Boca Raton, 2013; pp xiii-xxvi.

26. Kusalik, P. G.; Patey, G. N., On the Molecular Theory of Aqueous Electrolyte Solutions. II. Structural and Thermodynamic Properties of Different Models at Infinite Dilution. Journal of Chemical Physics 1988, 89, 5843-5851.

27. Stokes, R. H.; Robinson, R. A., Ionic Hydration and Activity in Electrolyte Solutions. Journal of the American Chemical Society 1948, 70, 1870-1878.

28. Bates, R. G.; Staples, B. R.; Robinson, R. A., Ionic Hydration and Single Ion Activities in Unassociated Chlorides at High Ionic Strengths. Analytical Chemistry 1970, 42, 867-\&.

29. Cruz, J.-L.; Renon, H., A New Thermodynamic Representation of Binary Electrolyte Solutions Nonideality in the Whole Range of Concentrations. AIChE Journal 1978, 24, 817.

30. Heyrovska, R., Degrees of Dissociation and Hydration Numbers of 26 Strong Electrolytes in Aqueous-Solutions at 25-Degrees-C. Collection of Czechoslovak Chemical Communications 1992, 57, 2209-2214.

31. Rabie, H. R.; Wilczek-Vera, G.; Vera, J. H., Activities of Individual Ions from Infinite Dilution to Saturated Solutions. Journal of Solution Chemistry 1999, 28, 885-913.

32. Frank, H. S.; Wen, W.-Y., Ion-Solvent Interaction. Structural Aspects of IonSolvent Interaction in Aqueous Solutions: A Suggested Picture of Water Structure. Discussions of the Faraday Society 1957, 24, 133-140.

33. Gurney, R. W., Ionic Processes in Solution; McGraw Hill: New York, 1953.

34. Frank, H. S.; Evans, M. W., Free Volume and Entropy in Condensed Systems III. Entropy in Binary Liquid Mixtures; Partial Molal Entropy in Dilute Solutions; Structure and Thermodynamics in Aqueous Electrolytes. The Journal of Chemical Physics 1945, 13, 507-532. 
35. Conway, B. E., Ionic Hydration in Chemistry and Biophysics; Elsevier: Amsterdam, 1981; Vol. 12.

36. Heyrovska, R., Physical Electrochemistry of Strong Electrolytes Based on Partial Dissociation and Hydration - Quantitative Interpretation of the Thermodynamic Properties of $\mathrm{NaCl}(\mathrm{Aq})$ from "Zero to Saturation". Journal of the Electrochemical Society 1996, 143, 1789-1793.

37. Burgess, J., Metal Ions in Solution; Ellis Horwood Limited: Chichester, 1978.

38. Marcus, Y., Ion Solvation; John Wiley \& Sons Ltd.: Chichester, 1985.

39. Chialvo, A. A.; Kusalik, P. G.; Cummings, P. T.; Simonson, J. M., Molecular Approach to High-Temperature Solvation. Formal, Integral Equations, and Experimental Results. Journal of Physics. Condensed Matter 2000, 12, 3585-3593.

40. Azzam, A. M., Theoretical Studies on Solvation: Part II. New Theory for Evaluation of Ionic Solvation Number for Divalent Ions at $25^{\circ} \mathrm{C}$. Canadian Journal of Chemistry 1960, 38, 993-1002.

41. Fabricand, B. P.; Leifer, R.; Ungar, S. G.; Goldberg, S. S., Proton Relaxation Times in Alkali Halide Solutions. Molecular Physics 1964, 7, 425-\&.

42. Ohtaki, H.; Radnai, T., Structure and Dynamics of Hydrated Ions. Chemical Review 1993, 93, 1157-1204.

43. Soper, A. K.; Neilson, G. W.; Enderby, J. E., Neutron Diffraction Study of Hydration Effects in Aqueous Solutions. Journal of Physics C. Solid State Physics 1977, 10, 1793-1801.

44. Enderby, J. E.; Nielsen, G. W., X-Ray and Neutron Scattering by Aqueous Solutions. In Water: A Comprehensive Treatise, Franks, F., Ed. Plenum Press: New York, 1979; Vol. 6, pp 1-46.

45. Soper, A. K.; Turner, J., Impact of Neutron Scattering on the Study of Water and Aqueous Solutions. International Journal of Modern Physics 1993, B7, 3049-3076.

46. Enderby, J. E., Ion Solvation Via Neutron Scattering. Chemical Society Review 1995, 24, 159-168.

47. Neilson, G. W.; Mason, P. E.; Ramos, S.; Sullivan, D., Neutron and X-Ray Scattering Studies of Hydration in Aqueous Solutions. Philosophical Transactions of the Royal Society a-Mathematical Physical and Engineering Sciences 2001, 359, 1575-1591.

48. Ansell, S.; Barnes, A. C.; Mason, P. E.; Neilson, G. W.; Ramos, S., X-Ray and Neutron Scattering Studies of the Hydration Structure of Alkali Ions in Concentrated Aqueous Solutions. Biophysical Chemistry 2006, 124, 171-179.

49. Kameda, Y.; Maki, A.; Amo, Y.; Usuki, T., Partial Pair Correlation Functions of Highly Concentrated Aqueous Urea Solutions Determined by Neutron Diffraction with N-14/N-15 and H/D Isotopic Substitution Methods. Bulletin of the Chemical Society of Japan 2010, 83, 131-144.

50. Soper, A. K., Neutron Scattering Studies of Solvent Structure in Systems of Chemical and Biological Importance. Faraday Discussions 1996, 103, 41-58.

51. Sears, V. F., Feature Section of Neutron Scattering Lengths and Cross Sections of the Elements and Their Isotopes. Neutron News 1992, 3, 29-37.

52. Dore, J. C.; Garawi, M.; Bellissent-Funel, M.-C., Neutron Diffraction Studies of the Structure of Water at Ambient Temperatures, Revisited. A Review of Past Developments and Currents Problems. Molecular Physics 2004, 102, 2015-2035. 
53. Chialvo, A. A.; Vlcek, L., "Thought Experiments" as Dry-Runs for "Tough Experiments": Novel Approaches to the Hydration Behavior of Oxyanions. The Journal of Physical Chemistry B 2015, In preparation.

54. Cartailler, T.; Kunz, W.; Turq, P.; Bellisent-Funel, M.-C., Lithium Bromide in Acetonitrile and Water: A Neutron Scattering Study. Journal of Physics. Condensed Matter 1991, 3, 9511-9520.

55. Kunz, W.; Barthel, J.; Klein, L.; Cartaillert, T.; Turq, P.; Reindl, B., Lithium Bromide in Acetonitrile - Thermodynamics, Theory, and Simulation. Journal of Solution Chemistry 1991, 20, 875-891.

56. Enderby, J. E.; Cummings, S.; Herdman, G. J.; Neilson, G. W.; Salmon, P. S.; Skipper, N., Diffraction and the Study of Aqua Ions. Journal of Physical Chemistry 1987, 91, 5851-5858.

57. Powell, D. H.; Neilson, G. W.; Enderby, J. E., A Neutron Diffraction Study of $\mathrm{NiCl}_{2}$ in $\mathrm{D}_{2} \mathrm{O}$ and $\mathrm{H}_{2} \mathrm{O}$. A Direct Determination of $\mathrm{G}_{\text {nih }}(\mathrm{R})$. Journal of Physics. Condensed Matter 1989, 1, 8721-8733.

58. Chialvo, A. A.; Simonson, J. M., The Structure of $\mathrm{CaCl}_{2}$ Aqueous Solutions over a Wide Range of Concentrations. Interpretation of Difraction Experiments Via Molecular Simulation. Journal of Chemical Physics 2003, 119, 8052-8061.

59. Chialvo, A. A.; Simonson, J. M., Ion Association in Aqueous LiCl Solutions at High Concentration: Predicted Results Via Molecular Simulation. Journal of Chemical Physics 2006, 124.

60. Placzek, G., The Scattering of Neutrons by Systems of Heavy Nuclei. Physical Review 1952, 86, 377-388.

61. Squires, G. L., Introduction to the Theory of Thermal Neutron Scattering; Dover Publications: Mineola, 1996.

62. Soper, A. K., The Radial Distribution Functions of Water as Derived from Radiation Total Scattering Experiments: Is There Anything We Can Say for Sure? ISRN Physical Chemistry 2013, 2013, 67.

63. Chialvo, A. A.; Vlcek, L., $\mathrm{NN}^{-}$Coordination in Aqueous Solutions by $15 \mathrm{~N} / 14 \mathrm{~N}$ and 180/natO Isotopic Substitution: What Can We Learn from Molecular Simulation? The Journal of Physical Chemistry B 2015, 119, 519-531.

64. Neilson, G. W.; Enderby, J. E., Aqueous Solutions and Neutron Scattering. Journal of Physical Chemistry 1996, 100, 1317-1322.

65. Enderby, J. E., Neutron-Diffraction, Isotopic-Substitution and the Structure of Aqueous-Solutions. Philosophical Transactions of the Royal Society of London Series B-Biological Sciences 1980, 290, 553-566.

66. Newsome, J. R.; Neilson, G. W.; Enderby, J. E.; Sandstrom, M., Ni ${ }^{2+}$ Hydration in Perchlorate and Chloride Solutions. Chemical Physics Letters 1981, 82, 399-401.

67. Neilson, G. W.; Enderby, J. E., The Structure of an Aqueous-Solution of Nickel Chloride. Proceedings of the Royal Society of London Series a-Mathematical Physical and Engineering Sciences 1983, 390, 353-371.

68. Powell, D. H.; Neilson, G. W., The Concentration Dependence of the $\mathrm{Ni}^{+2}$ Hydration Geometry in Aqueous Solution. Journal of Physics. Condensed Matter 1990, 2, 3871-3878. 
69. Powell, D. H.; Neilson, G. W.; Enderby, J. E., The Structure of $\mathrm{Cl}^{-}$in Aqueous Solution: An Experimental Determination of $\mathrm{G}_{\mathrm{ClH}}(\mathrm{R})$ and $\mathrm{G}_{\mathrm{ClO}}(\mathrm{R})$. Journal of Physics: Condensed Matter 1993, 5, 5723-5730.

70. Howell, I.; Neilson, G. W., Li+ Hydration in Concentrated Aqueous Solutions. Journal of Physics: Condensed Matter 1996, 8, 4455-4463.

71. Chialvo, A. A.; Simonson, J. M., The Structure of Concentrated $\mathrm{NiCl}_{2}$ Aqueous Solutions. What Is Molecular Simulation Revealing About the Neutron Scattering Methodologies? Molecular Physics 2002, 100, 2307-2315.

72. Powell, D. H.; Barnes, A. C.; Enderby, J. E.; Neilson, G. W.; Salmon, P. S., The Hydration Structure around Chloride Ions in Aqueous Solution. Faraday Discussions 1988, 85, 137-146.

73. Megyes, T.; Bako, I.; Balint, S.; Grosz, T.; Radnai, T., Ion Pairing in Aqueous Calcium Chloride Solution: Molecular Dynamics Simulation and Diffraction Studies. Journal of Molecular Liquids 2006, 129, 63-74.

74. Phutela, R. C.; Pitzer, K. S., Thermodynamics of Aqueous Calcium-Chloride. Journal of Solution Chemistry 1983, 12, 201-207.

75. Williamsjones, A. E.; Seward, T. M., The Stability of Calcium-Chloride Ion-Pairs in Aqueous-Solutions at Temperatures between 100-Degrees-C and 360-Degrees-C. Geochimica et Cosmochimica Acta 1989, 53, 313-318.

76. Holmes, H. F.; Busey, R. H.; Simonson, J. M.; Mesmer, R. E., CaCl2(Aq) at Elevated-Temperatures - Enthalpies of Dilution, Isopiestic Molalities, and Thermodynamic Properties. Journal of Chemical Thermodynamics 1994, 26, 271298.

77. Rard, J. A.; Clegg, S. L., Critical Evaluation of the Thermodynamic Properties of Aqueous Calcium Chloride .1. Osmotic and Activity Coefficients of 0-10.77 Mol Center Dot $\mathrm{Kg}(-1)$ Aqueous Calcium Chloride Solutions at 298.15 K and Correlation with Extended Pitzer Ion-Interaction Models. Journal of Chemical and Engineering Data 1997, 42, 819-849.

78. Pitzer, K. S.; Wang, P. M.; Rard, J. A.; Clegg, S. L., Thermodynamics of Electrolytes. 13. Ionic Strength Dependence of Higher-Order Terms; Equations for $\mathrm{CaCl} 2$ and $\mathrm{MgCl}$ 2. Journal of Solution Chemistry 1999, 28, 265-282.

79. Oscarson, J. L.; Gillespie, S. E.; Chen, X. M.; Schuck, P. C.; Izatt, R. M., Enthalpies of Dilution of Aqueous Solutions of $\mathrm{HCl}, \mathrm{MgCl} 2, \mathrm{CaCl} 2$, and $\mathrm{BaCl} 2$ at 300, 325, and 350 Degrees C. Journal of Solution Chemistry 2001, 30, 31-53.

80. Zeng, D. W.; Zhou, H. Y.; Voigt, W., Thermodynamic Consistency of Solubility and Vapor Pressure of a Binary Saturated Salt Plus Water System - II. CaCl2+H2O. Fluid Phase Equilibria 2007, 253, 1-11.

81. Cummings, S.; Enderby, J. E.; Howe, R. A., Ion Hydration in Aqueous $\mathrm{CaCl}_{2}$ Solutions. Journal of Physics C: Solid State Physics 1980, 13, 1-8.

82. Hewish, N. A.; Neilson, G. W.; Enderby, J. E., Environment of $\mathrm{Ca}^{+2}$ Ions in Aqueous Solvent. Nature 1982, 297, 138-139.

83. Fulton, J. L.; Heald, S. M.; Badyal, Y. S.; Simonson, J. M., Understanding the Effects of Concentration on the Solvation Structure of $\mathrm{Ca}^{+2}$ in Aqueous Solution. I. The Perspective on Local Structure from EXAFS and XANES. Journal of Physical Chemistry A 2003, 107, 4688-4696. 
84. Badyal, Y. S.; Barnes, A. C.; Cuello, G. J.; Simonson, J. M., Understanding the Effects of Concentration on the Solvation Structure of $\mathrm{Ca} 2+$ in Aqueous Solutions. Ii: Insights into Longer Range Order from Neutron Diffraction Isotope Substitution. Journal of Physical Chemistry A 2004, 108, 11819-11827.

85. Bruni, F.; Imberti, S.; Mancinelli, R.; Ricci, M. A., Aqueous Solutions of Divalent Chlorides: Ions Hydration Shell and Water Structure. Journal of Chemical Physics 2012, 136.

86. Albright, J. N., X-Ray Diffraction Studies of Aqueous Alkaline-Earth Chloride Solutions. Journal of Chemical Physics 1972, 56, 3783-3786.

87. Licheri, G.; Piccaluga, G.; Pinna, G., X-Ray Diffraction Study of the Average Solute Species in $\mathrm{Cacl}_{2}$ Aqueous Solutions. Journal of Chemical Physics 1976, 64, 2437-2441.

88. Caminiti, R.; Licheri, G.; Paschina, G.; Piccaluga, G.; Pinna, G., X-Ray-Diffraction and Structural-Properties of Aqueous-Solutions of Divalent Metal-Chlorides. Zeitschrift Fur Naturforschung Section a-a Journal of Physical Sciences 1980, 35, 1361-1367.

89. Yamaguchi, T.; Hayashi, S.; Ochiai, H., X-Ray Diffraction Study of Calcium(II) Chloride Hydrate Melts: $\mathrm{CaCl}_{2} \cdot \mathrm{rH}_{2} \mathrm{O}(\mathrm{r}=4.0,5.6,6.0,8.6)$. Inorganic Chemistry 1989, 28, 2434-2439.

90. Probst, M. M.; Radnai, T.; Heinzinger, K. Z., Molecular Dynamics and X-Ray Investigation of the Aqueous $\mathrm{CaCl}_{2}$ Solution. Journal of Physical Chemistry 1985, 89, 753-759.

91. Gaspar, A. M.; Marques, M. A.; Cabaco, M. I.; Marques, M. I. D.; Buslaps, T.; Honkimaki, V., X-Ray Diffraction Investigations of Concentrated Aqueous Solutions of Calcium Halides. Journal of Molecular Liquids 2004, 110, 15-22.

92. Megyes, T.; Grosz, T.; Radnai, T.; Bako, I.; Palinkas, G., Solvation of Calcium Ion in Polar Solvents: An X-Ray Diffraction and Ab Initio Study. Journal of Physical Chemistry A 2004, 108, 7261-7271.

93. Pham, V.-T.; Fulton, J. L., Ion-Pairing in Aqueous $\mathrm{CaCl}[\mathrm{Sub} 2]$ and $\mathrm{RbBr}$ Solutions: Simultaneous Structural Refinement of XAFS and XRD Data. The Journal of Chemical Physics 2013, 138, 044201-044213.

94. Spångberg, D.; Hermansson, K.; Lindqvist-Reis, P.; Jalilehvand, F.; Sandström, M., Model Extended X-Ray Absorption Fine Structure (EXAFS) Spectra from Molecular Dynamics Dta for $\mathrm{Ca}+2$ and $\mathrm{Al}+3$ Aqueous Solutions. Journal of Physical Chemistry B 2000, 104, 10467-10472.

95. Jalilehvand, F.; Spångberg, D.; Lindqvist-Reis, P.; Hermansson, K.; Perssom, I.; Sandström, M., Hydration of the Calcium Ion. An Exafs, Large-Angle X-Ray Scattering, and Molecular Dynamics Simulation Study. Journal of the American Chemical Society 2001, 123, 431-441.

96. Fulton, J. L.; Chen, Y.; Heald, S. M.; Balasubramanian, M., Hydration and Contact Ion Pairing of Ca2+ with Cl- in Supercritical Aqueous Solution. Journal of Chemical Physics 2006, 125.

97. Tongraar, A.; T-Thienprasert, J.; Rujirawat, S.; Limpijumnong, S., Structure of the Hydrated Ca2+ and Cl-: Combined X-Ray Absorption Measurements and Qm/Mm Md Simulations Study. Physical Chemistry Chemical Physics 2010, 12, 1087610887. 
98. Frantz, J. D.; Marshall, W. L., Electrical Conductances and Ionization-Constants of Calcium-Chloride and Magnesium-Chloride in Aqueous-Solutions at Temperatures to 600-Degrees-C and Pressures to 4000 Bars. American Journal of Science 1982, 282, 1666-1693.

99. Ribeiro, A. C. F.; Barros, M. C. F.; Teles, A. S. N.; Valente, A. J. M.; Lobo, V. M. M.; Sobral, A. J. F. N.; Esteso, M. A., Diffusion Coefficients and Electrical Conductivities for Calcium Chloride Aqueous Solutions at 298.15K and 310.15K. Electrochimica Acta 2008, 54, 192-196.

100. Guàrdia, E.; Robinson, A.; Padró, J. A., Mean Force Potential for the CalciumChloride Ion Pair in Water. Journal of Chemical Physics 1993, 99, 4229-4230.

101. Dang, L. X.; Smith, D. E., Comment on "Mean Force Potential for the CalciumChloride Ion Pair in Water" [J. Chem. Phys. 99, 4229 (1993)]. Journal of Chemical Physics 1995, 102, 3483-3484.

102. Lee, S. H., Molecular Dynamics Simulation Studies of the Limiting Conductances of $\mathrm{CaCl} 2$ Using Extended Simple Point Charge and Revised Polarizable Models. Molecular Simulation 2004, 30, 669-678.

103. Li, M. Y.; Duan, Z. H.; Zhang, Z. G.; Zhang, C.; Weare, J., The Structure, Dynamics and Solvation Mechanisms of Ions in Water from Long Time Molecular Dynamics Simulations: A Case Study of $\mathrm{CaCl} 2$ (Aq) Aqueous Solutions. Molecular Physics 2008, 106, 2685-2697.

104. Todorova, T.; Hunenberger, P. H.; Hutter, J., Car-Parrinello Molecular Dynamics Simulations of $\mathrm{CaCl}(2)$ Aqueous Solutions. Journal of Chemical Theory and Computation 2008, 4, 779-789.

105. Timko, J.; De Castro, A.; Kuyucak, S., Ab Initio Calculation of the Potential of Mean Force for Dissociation of Aqueous Ca-Cl. Journal of Chemical Physics 2011, 134.

106. Dang, L. X.; Truong, T. B.; Ginovska-Pangovska, B., Note: Interionic Potentials of Mean Force for Ca2+-Cl- in Polarizable Water. Journal of Chemical Physics 2012, 136.

107. Pitzer, K. S., Thermodynamics of Electrolytes .1. Theoretical Basis and General Equations. Journal of Physical Chemistry 1973, 77, 268-277.

108. Pham, V. T.; Fulton, J. L., Ion-Pairing in Aqueous CaCl2 and Rbbr Solutions: Simultaneous Structural Refinement of Xafs and Xrd Data. Journal of Chemical Physics 2013, 138.

109. Dang, L. X.; Truong, T. B.; Ginovska-Pangovska, B., Note: Interionic Potentials of Mean Force for $\mathrm{Ca}^{2+}-\mathrm{Cl}^{-}$in Polarizable Water. The Journal of Chemical Physics 2012, 136, 126101.

110. Babu, C. S.; Lim, C., Empirical Force Fields for Biologically Active Divalent Metal Cations in Water. Journal of Physical Chemistry A 2006, 110, 691-699.

111. Mamatkulov, S.; Fyta, M.; Netz, R. R., Force Fields for Divalent Cations Based on Single-Ion and Ion-Pair Properties. The Journal of Chemical Physics 2013, 138, 024505-024512.

112. Howe, R. A.; Howells, W. S.; Enderby, J. E., Ion Distribution and Long Range Order in Concentrated Electrolyte Solutions. Journal of Physics C. Solid State Physics 1974, 7, L111-114. 
113. Enderby, J. E., Neutron and X-Ray Scattering from Aqueous Solutions. Proceedings of the Royal Society of London 1975, A 345, 107-117.

114. Badyal, Y. S.; Simonson, J. M.; Annis, B. K.; Londono, J. D., The Hydration Structure of $\mathrm{Ni}^{+2}$ in Aqueous Solution by Neutron Diffraction Isotope Substitution: A Case Study on the Effects of Systematic Error. Journal of Neutron Research 2002, 10, 19-29.

115. Caminiti, R.; Licheri, G.; Paschina, G.; Piccaluga, G.; Pinna, G., X-Ray Diffraction and Structural Properties of Aqueous Solutions of Divalent Metal-Chlorides. $Z$. Naturforsch 1980, 35a, 1361-1367.

116. Caminiti, R.; Licheri, G.; Piccaluga, G.; Pinna, G., X-Ray-Diffraction and Structure of NiCl2 Aqueous-Solutions. Faraday Discussions 1977, 64, 62-68.

117. Fontana, M. P.; Maisano, G.; Migliardo, P.; Wanderlingh, F., Raman-Spectroscopy and Local Order in Aqueous-Solutions of Strong II-I Electrolytes. Journal of Chemical Physics 1978, 69, 676-683.

118. Sandstrom, D. R., Ni2+ Coordination in Aqueous NiCl2 Solutions - Study of the Extended X-Ray Absorption Fine-Structure. Journal of Chemical Physics 1979, 71, 2381-2386.

119. Aziz, E. F.; Eisebitt, S.; de Groot, F.; Chiou, J. W.; Dong, C. G.; Guo, J. H.; Eberhardt, W., Direct Contact Versus Solvent-Shared Ion Pairs in NiCl2 Electrolytes Monitored by Multiplet Effects at Ni(II) L Edge X-Ray Absorption. Journal of Physical Chemistry B 2007, 111, 4440-4445.

120. Magini, M., Hydration and Complex-Formation Study on Concentrated-Solutions $\mathrm{Co}(11) \mathrm{Cl} 2 \mathrm{Ni}(11) \mathrm{Cl} 2 \mathrm{Cu}(11) \mathrm{Cl} 2$ by X-Ray-Diffraction Technique. Journal of Chemical Physics 1981, 74, 2523-2529.

121. Magini, M.; Paschina, G.; Piccaluga, G., Ni-Cl Bonding in Concentrated Ni(II) Aqueous-Solutions at High Cl-Ni2+ Ratios - an X-Ray-Diffraction Investigation. Journal of Chemical Physics 1982, 76, 1116-1121.

122. Licheri, G.; Paschina, G.; Piccaluga, G.; Pinna, G., Exafs Study of Ni-Cl Bonding in $\mathrm{Ni}$ (II) Aqueous-Solutions at Increasing Cl-/Ni2+ Ratios. Journal of Chemical Physics 1983, 79, 2168-2171.

123. Waizumi, K.; Kouda, T.; Tanio, A.; Fukushima, N.; Ohtaki, H., Structural Studies on Saturated Aqueous Solutions of Manganese(II), Cobalt(II), and Nickel(II) Chlorides by X-Ray Diffraction. Journal of Solution Chemistry 1999, 28, 83-100.

124. Weingärtner, H.; Muller, C.; Hertz, H. G., Composition of the 1st Coordination Sphere of $\mathrm{Ni2}+$ in Concentrated Aqueous NiCl2 and NiBr2 Solutions .2. Application of Halide Nuclear Magnetic-Relaxation. Journal of the Chemical Society-Faraday Transactions I 1979, 75, 2712-2734.

125. Sharma, V.; Bohm, F.; Schwaab, G.; Havenith, M., The Low Frequency Motions of Solvated Mn(II) and Ni(II) Ions and Their Halide Complexes. Physical Chemistry Chemical Physics 2014, 16, 25101-25110.

126. Cummings, S.; Enderby, J. E.; Neilson, G. W.; Newsome, J. R.; Howe, R. A.; Howells, W. S.; Soper, A. K., Chloride Ions in Aqueous Solutions. Nature 1980, 287, 714-716.

127. Ohtomo, N.; Arakawa, K., Neutron-Diffraction Study of Aqueous Ionic-Solutions .1. Aqueous-Solutions of Lithium-Chloride and Cesium-Chloride. Bulletin of the Chemical Society of Japan 1979, 52, 2755-2759. 
128. Newsome, J. R.; Neilson, G. W.; Enderby, J. E., Lithium Ions in Aqueous Solution. Journal of Physics C: Solid State Physics 1980, 13, L923-L926.

129. Ichikawa, K.; Kameda, Y.; Matsumoto, T.; Misawa, M., Indirect and Direct Correlations between Unlike Ions in Incompletely Hydrated Solution. Journal of Physics C: Solid State Physics 1984, 17, L725-L729.

130. Copestake, A. P.; Neilson, G. W.; Enderby, J. E., The Structure of a Highly Concentrated Aqueous-Solution of Lithium-Chloride. Journal of Physics C-Solid State Physics 1985, 18, 4211-4216.

131. Ichikawa, K.; Kameda, Y., The Intra-Molecular Structure of a Water Molecule in Hydrated and Incompletely Hydrated LiCl Solutions. Journal of Physics-Condensed Matter 1989, 1, 257-266.

132. Tromp, R. H.; Neilson, G. W.; Soper, A. K., Water Structure in Concentrated Lithium Chloride Solutions. Journal of Chemical Physics 1992, 96, 8460-8469.

133. Ansell, S.; Neilson, G. W., Anion-Anion Pairing in Concentrated Aqueous Lithium Chloride Solution. Journal of Chemical Physics 2000, 112, 3942-3944.

134. Yamaguchi, T.; Ohzono, H.; Yamagami, M.; Yamanaka, K.; Yoshida, K.; Wakita, H., Ion Hydration in Aqueous Solutions of Lithium Chloride, Nickel Chloride, and Caesium Chloride in Ambient to Supercritical Water. Journal of Molecular Liquids 2010, 153, 2-8.

135. Winkel, K.; Seidl, M.; Loerting, T.; Bove, L. E.; Imberti, S.; Molinero, V.; Bruni, F.; Mancinelli, R.; Ricci, M. A., Structural Study of Low Concentration LiCl Aqueous Solutions in the Liquid, Supercooled, and Hyperquenched Glassy States. Journal of Chemical Physics 2011, 134.

136. Mason, P. E.; Ansell, S.; Neilson, G. W.; Rempe, S. B., Neutron Scattering Studies of the Hydration Structure of Li+. The Journal of Physical Chemistry B 2015, 119, 2003-2009.

137. Brady, G. W., Structure in Ionic Solutions .2. Journal of Chemical Physics 1958, 28, 464-469.

138. Lawrence, R. M.; Kruh, R. F., X-Ray Diffraction Studies of Aqueous Alkali-Metal Halide Solutions. Journal of Chemical Physics 1967, 47, 4758-\&.

139. Narten, A. H.; Vaslow, F.; Levy, H. A., Diffraction Pattern and Structure of Aqueous Lithium-Chloride Solutions. Journal of Chemical Physics 1973, 58, 5017 5023.

140. Yamanaka, K.; Yamagami, M.; Takamuku, T.; Yamaguchi, T.; Wakita, H., X-RayDiffraction Study on Aqueous Lithium-Chloride Solution in the TemperatureRange 138-373-K. Journal of Physical Chemistry 1993, 97, 10835-10839.

141. Bouazizi, S.; Nasr, S., Structural Investigations of High Concentrated Aqueous LiCl Solutions: X-Ray Scattering and Md Simulations Approach. Journal of Molecular Structure 2008, 875, 121-129.

142. Harsanyi, I.; Temleitner, L.; Beuneu, B.; Pusztai, L., Neutron and X-Ray Diffraction Measurements on Highly Concentrated Aqueous LiCl Solutions. Journal of Molecular Liquids 2012, 165, 94-100.

143. Seth, A.; Baerends, E. J., Compton Profiles of Aqueous-Solutions of LiCl. Chemical Physics Letters 1979, 64, 165-169. 
144. Nygård, K.; Hakala, M.; Manninen, S.; Hämäläinen, K.; Itou, M.; Andrejczuk, A.; Sakurai, Y., Ion Hydration Studied by X-Ray Compton Scattering. Physical Review B 2006, 73, 024208.

145. Max, J. J.; Chapados, C., Ir Spectroscopy of Aqueous Alkali Halide Solutions: Pure Salt-Solvated Water Spectra and Hydration Numbers. Journal of Chemical Physics 2001, 115, 2664-2675.

146. David, R.; Henry, F., Study of Ion Hydration from Dielectric Mixture Formula by Microwave Spectrometry. Journal de Chimie Physique et de Physico-Chimie Biologique 1986, 83, 527-532.

147. Barthel, J.; Schmitha.F; Behret, H., Studies on Dispersion of Complex Dielectricity Constants of Aqueous and Non-Aqueous Electrolyte Solutions .1. Choice of Measuring Methods and Measurement on Aqueous Alkaline Chloride and Alkaline Nitrate Solutions up to Saturation Concentration at 25 Degrees C in Range of Cm Waves. Zeitschrift Fur Physikalische Chemie-Frankfurt 1970, 71, 115-\&.

148. Wei, Y. Z.; Sridhar, S., Dielectric-Spectroscopy up to $20 \mathrm{Ghz}$ of $\mathrm{LiCl} / \mathrm{H} 2 \mathrm{O}$ Solutions. Journal of Chemical Physics 1990, 92, 923-928.

149. Wachter, W.; Fernandez, S.; Buchner, R.; Hefter, G., Ion Association and Hydration in Aqueous Solutions of $\mathrm{LiCl}$ and $\mathrm{Li} 2 \mathrm{SO} 4$ by Dielectric Spectroscopy. Journal of Physical Chemistry B 2007, 111, 9010-9017.

150. Jepsen, P. U.; Merbold, H., Terahertz Reflection Spectroscopy of Aqueous $\mathrm{NaCl}$ and LiCl Solutions. Journal of Infrared Millimeter and Terahertz Waves 2010, 31 , 430-440.

151. Green, J. L.; Lacey, A. R.; Sceats, M. G., Determination of the Total Hydration Number of a LiCl Cation Anion Pair Via Collective Proton Motions. Chemical Physics Letters 1987, 134, 385-391.

152. Nash, C. P.; Donnelly, T. C.; Rock, P. A., Raman-Spectra in Libration Region of Concentrated Aqueous-Solutions of Li-6 and Li-7 Halides - Evidence for Tetrahedral Li(OH2)4+. Journal of Solution Chemistry 1977, 6, 663-670.

153. Hasebe, T.; Tamamushi, R.; Tanaka, K., Nuclear-Magnetic-Resonance and Thermal Studies of Concentrated Aqueous-Solutions of Lithium-Chloride. Journal of the Chemical Society-Faraday Transactions 1992, 88, 205-208.

154. Tongraar, A.; Liedl, K. R.; Rode, B. M., The Hydration Shell Structure of Li+ Investigated by Born-Oppenheimer Ab Initio Qm/Mm Dynamics. Chemical Physics Letters 1998, 286, 56-64.

155. Rempe, S. B.; Pratt, L. R.; Hummer, G.; Kress, J. D.; Martin, R. L.; Redondo, A., The Hydration Number of $\mathrm{Li}(+)$ in Liquid Water. Journal of the American Chemical Society 2000, 122, 966-967.

156. Lyubartsev, A. P.; Laasonen, K.; Laaksonen, A., Hydration of Li+ Ion. An Ab Initio Molecular Dynamics Simulation. Journal of Chemical Physics 2001, 114, 31203126.

157. Loeffler, H. H.; Rode, B. M., The Hydration Structure of the Lithium Ion. Journal of Chemical Physics 2002, 117, 110-117.

158. Degreve, L.; Mazze, F. M., Molecular Simulation of LiCl Aqueous Solutions. Molecular Physics 2003, 101, 1443-1453.

159. Egorov, A. V.; Komolkin, A. V.; Chizhik, V. I.; Yushmanov, P. V.; Lyubartsev, A. P.; Laaksonen, A., Temperature and Concentration Effects on Li+-Ion Hydration. A 
Molecular Dynamics Simulation Study. Journal of Physical Chemistry B 2003, 107, 3234-3242.

160. Varma, S.; Rempe, S. B., Coordination Numbers of Alkali Metal Ions in Aqueous Solutions. Biophysical Chemistry 2006, 124, 192-199.

161. Chialvo, A. A.; Simonson, J. M., Ion Pairing and Counterion Condensation in Aqueous Electrolyte and Polyelectrolyte Solutions: Insights from Molecular Simulation. Journal of Molecular Liquids 2007, 134, 15-22.

162. Du, H.; Rasaiah, J. C.; Miller, J. D., Structural and Dynamic Properties of Concentrated Alkali Halide Solutions: A Molecular Dynamics Simulation Study. Journal of Physical Chemistry B 2007, 111, 209-217.

163. Petit, L.; Vuilleumier, R.; Maldivi, P.; Adamo, C., Ab Initio Molecular Dynamics Study of a Highly Concentrated LiCl Aqueous Solution. J. Chem. Theory Comput. 2008.

164. Harsanyi, I.; Pusztai, L., Hydration Structure in Concentrated Aqueous Lithium Chloride Solutions: A Reverse Monte Carlo Based Combination of Molecular Dynamics Simulations and Diffraction Data. The Journal of Chemical Physics 2012, 137, 204503-204509.

165. Bouazizi, S.; Nasr, S., Concentration Effects on Aqueous Lithium Chloride Solutions. Molecular Dynamics Simulations and X-Ray Scattering Studies. Journal of Molecular Liquids 2014, 197, 77-83.

166. Xu, J.-J.; Yi, H.-B.; Li, H.-J.; Chen, Y., Ionic Solvation and Association in LiCl Aqueous Solution: A Density Functional Theory, Polarised Continuum Model and Molecular Dynamics Investigation. Molecular Physics 2014, 112, 1710-1723.

167. Harsanyi, I.; Pusztai, L., On the Structure of Aqueous LiCl Solutions. Journal of Chemical Physics 2005, 122.

168. Rudolph, W.; Brooker, M. H.; Pye, C. C., Hydration of Lithium Ion in AqueousSolution. Journal of Physical Chemistry 1995, 99, 3793-3797.

169. Fischer, H. E.; Simonson, J. M.; Neuefeind, J. C.; Lemmel, H.; Rauch, H.; Zeidler, A.; Salmon, P. S., The Bound Coherent Neutron Scattering Lengths of the Oxygen Isotopes. Journal of Physics-Condensed Matter 2012, 24.

170. Zeidler, A.; Salmon, P. S.; Fischer, H. E.; Neuefeind, J. C.; Simonson, J. M.; Markland, T. E., Isotope Effects in Water as Investigated by Neutron Diffraction and Path Integral Molecular Dynamics. Journal of Physics-Condensed Matter 2012, 24.

171. Hefter, G., When Spectroscopy Fails: The Measurement of Ion Pairing. Pure and Applied Chemistry 2006, 78, 1571-1586.

172. Caminiti, R.; Licheri, G.; Piccaluga, G.; Pinna, G., NO-3-H2O Interactions in Aqueous-Solutions. Journal of Chemical Physics 1978, 68, 1967-1970.

173. Caminiti, R.; Cucca, P.; Radnai, T., Investigation on the Structure of Cadmium Nitrate Aqueous-Solutions by X-Ray-Diffraction and Raman-Spectroscopy. Journal of Physical Chemistry 1984, 88, 2382-2386.

174. Irish, D. E.; Davis, A. R.; Plane, R. A., Types of Interaction in Some Aqueous Metal Nitrate Systems. Journal of Chemical Physics 1969, 50, 2262-\&.

175. Irish, D. E.; Chang, G.; Nelson, D. L., Concerning Cation-Nitrate Ion Contact in Aqueous Solutions. Inorganic Chemistry 1970, 9, 425-\&. 
176. Vogrin, F. J.; Knapp, P. S.; Flint, W. L.; Anton, A.; Highberger, G.; Malinowski, E. R., Nmr Studies of Aqueous Electrolyte Solutions. Iv. Hydration Numbers of Strong Electrolytes Determined from Temperature Effects on Proton Shifts. Journal of Chemical Physics 1971, 54, 178-181.

177. Chizhik, V. I., Nmr Relaxation and Microstructure of Aqueous Electrolyte Solutions. Molecular Physics 1997, 90, 653-659.

178. Nicholas, A. M. D.; Wasylishen, R. E., A Nuclear-Magnetic-Resonance Study of Aqueous-Solutions of Several Nitrate Salts. Canadian Journal of Chemistry-Revue Canadienne De Chimie 1987, 65, 951-956.

179. Adachi, A.; Kiyoyama, H.; Nakahara, M.; Masuda, Y.; Yamatera, H.; Shimizu, A.; Taniguchi, Y., Raman and Nuclear Magnetic-Resonance Studies on the Concentration-Dependence of Orientational Relaxation-Times of the Nitrate Ion in Dilute Aqueous-Solution. Journal of Chemical Physics 1989, 90, 392-399.

180. Owens, G.; Guarilloff, P.; Steel, B. J.; Kurucsev, T., N-14 Nuclear-MagneticResonance Relaxation of the Nitrate Ion and Ion-Pairing in Aqueous-Solution. Australian Journal of Chemistry 1995, 48, 207-215.

181. Choppin, G. R.; Buijs, K., Near-Infrared Studies of Structure of Water .2. Ionic Solutions. Journal of Chemical Physics 1963, 39, 2042-\&.

182. Sze, Y. K.; Irish, D. E., Vibrational Spectral Studies of Ion-Ion and Ion-Solvent Interactions .1. Zinc Nitrate in Water. Journal of Solution Chemistry 1978, 7, 395415.

183. Bergstrom, P. A.; Lindgren, J.; Kristiansson, O., An Ir Study of the Hydration of ClO4-, NO3-, I-, Br-, Cl-, and SO42- Anions in Aqueous-Solution. Journal of Physical Chemistry 1991, 95, 8575-8580.

184. Liu, J. H.; Zhang, Y. H.; Wang, L. Y.; Wei, Z. F., Drawing out the Structural Information of the First Layer of Hydrated Ions: Atr-Ftir Spectroscopic Studies on Aqueous NH4NO3, NaNO3, and $\mathrm{Mg}(\mathrm{NO} 3)(2)$ Solutions. Spectrochimica Acta Part a-Molecular and Biomolecular Spectroscopy 2005, 61, 893-899.

185. Goebbert, D. J.; Garand, E.; Wende, T.; Bergmann, R.; Meijer, G.; Asmis, K. R.; Neumark, D. M., Infrared Spectroscopy of the Microhydrated Nitrate Ions NO3(H2O)(1-6). Journal of Physical Chemistry A 2009, 113, 7584-7592.

186. Shaffer, C. J.; Schroder, D., Microhydrated Cobalt-Nitrate Cations $\mathrm{Co}(\mathrm{NO} 3)(\mathrm{H} 2 \mathrm{O})(\mathrm{N})(+)(\mathrm{N}=2,3)$ Studied by Infrared Spectroscopy in the Gas Phase. International Journal of Mass Spectrometry 2012, 311, 17-23.

187. Fleissner, G.; Hallbrucker, A.; Mayer, E., $\mathrm{Nu}(2)$ Band Region of Nitrate as an Indicator for Contact Ion Pairing in Aqueous Lithium and Calcium Nitrate Solutions. Journal of the Chemical Society-Faraday Transactions 1996, 92, 23-28.

188. Vollmar, P. M., Ionic Interactions in Aqueous Solution: A Raman Spectral Study. The Journal of Chemical Physics 1963, 39, 2236-2248.

189. Peleg, M., Raman Spectroscopic Investigation of Magnesium Nitrate-Water System. Journal of Physical Chemistry 1972, 76, 1019-\&.

190. Caminiti, R.; Atzei, D.; Cucca, P.; Anedda, A.; Bongiovanni, G., Structure of Rhodium(III) Nitrate Aqueous-Solutions - an Investigation by X-Ray-Diffraction and Raman-Spectroscopy. Journal of Physical Chemistry 1986, 90, 238-243. 
191. Waterland, M. R.; Stockwell, D.; Kelley, A. M., Symmetry Breaking Effects in NO3-: Raman Spectra of Nitrate Salts and Ab Initio Resonance Raman Spectra of Nitrate-Water Complexes. Journal of Chemical Physics 2001, 114, 6249-6258.

192. Zhang, Y. H.; Choi, M. Y.; Chan, C. K., Relating Hygroscopic Properties of Magnesium Nitrate to the Formation of Contact Ion Pairs. Journal of Physical Chemistry A 2004, 108, 1712-1718.

193. Marques, M. A.; Marques, M. I. D.; Cabaco, M. I.; Gaspar, A. M.; de Almeida, M. L., Intermediate Range Order in Concentrated Aqueous Solutions of Copper Nitrate. X-Ray Diffraction and Raman Investigations. Journal of Molecular Liquids 2004, 110, 23-31.

194. Rohman, N.; Wahab, A.; Mahiuddin, S., Isentropic Compressibility, Shear Relaxation Time, and Raman Spectra of Aqueous Calcium Nitrate and Cadmium Nitrate Solutions. Journal of Solution Chemistry 2005, 34, 77-94.

195. Cabaco, M. I.; Marques, M. I. D.; Marques, M. A.; Gaspar, A. M.; de Almeida, M. L., X-Ray Diffraction and Raman Spectroscopy Investigations in Concentrated Aqueous Solutions of Yttrium and Strontium Nitrates. Journal of Molecular Liquids 2005, 117, 69-76.

196. Marques, M. A.; Marques, M. I. D.; Cabaco, M. I.; Gaspar, A. M.; Marques, M. P. M.; Amado, A. M.; da Costa, A. M. A., Evidence of a Local Order in Concentrated Aqueous Solutions of Salts Constituted by Ions of Different Valences. X-Ray Diffraction and Raman Spectroscopy Experiments. Journal of Molecular Liquids 2007, 134, 142-150.

197. Cabaco, M. I.; Marques, M.; Gaspar, A. M.; Marques, M. A.; Costa, M. M., The Structure of Concentrated Aqueous Solutions of Chromium Nitrate and Cerium Chloride Studied by X-Ray Diffraction and Raman Spectroscopy. Journal of Molecular Liquids 2007, 136, 323-330.

198. Caminiti, R.; Licheri, G.; Paschina, G.; Piccaluga, G.; Pinna, G., Interactions and Structure in Aqueous NaNO3 Solutions. Journal of Chemical Physics 1980, 72, $4522-4528$.

199. Dagnall, S. P.; Hague, D. N.; Towl, A. D. C., X-Ray-Diffraction Study of Aqueous Zinc(II) Nitrate. Journal of the Chemical Society-Faraday Transactions II 1982, 78, 2161-2167.

200. Valeev, A. K.; Smirnov, P. R.; Trostin, V. N.; Krestov, G. A., Characteristics of Nitrate-Ion Hydration in Aqueous-Solutions in Nitric-Acid. Russian Journal of Physical Chemistry 1988, 62, 155-157.

201. Burke, C. A. E.; Adya, A. K.; Neilson, G. W., A Structural Study of the AquaAnions NO3-, BrO3- and ClO3- by Difference-Methods of X-Ray-Diffraction. Journal of Physics-Condensed Matter 1991, 3, 837-850.

202. Smirnov, P.; Yamagami, M.; Wakita, H.; Yamaguchi, T., An X-Ray Diffraction Study on Concentrated Aqueous Calcium Nitrate Solutions at Subzero Temperatures. Journal of Molecular Liquids 1997, 73-4, 305-316.

203. Levochkin, S. F.; Smirnov, P. R.; Trostin, V. N., D-Structure of Aqueous Solutions of Cobalt(II) Nitrate at 298 And. 323 K by X-Ray Diffraction. Russian Journal of General Chemistry 2005, 75, 1180-1185. 
204. Smirnov, P. R.; Grechin, O. V.; Trostin, V. N., Concentration Dependence of the Structure of Aqueous Solutions of Lutetium Nitrate According to X-Ray Diffraction. Russian Journal of Physical Chemistry A 2014, 88, 250-253.

205. Neilson, G. W.; Enderby, J. E., The Structure around Nitrate Ions in Concentrated Aqueous-Solutions. Journal of Physics C-Solid State Physics 1982, 15, 2347-2352.

206. Walker, P. A. M.; Lawrence, D. G.; Neilson, G. W.; Cooper, J., The Structure of Concentrated Aqueous Ammonium-Nitrate Solutions. Journal of the Chemical Society-Faraday Transactions I 1989, 85, 1365-1372.

207. Adya, A. K.; Neilson, G. W., Structure of a $50 \mathrm{Mol} \mathrm{Kg-1} \mathrm{Aqueous-Solution} \mathrm{of}$ Ammonium-Nitrate at 373-K by the Isotopic Difference Method of NeutronDiffraction. Journal of the Chemical Society-Faraday Transactions 1991, 87, 279286.

208. Kameda, Y.; Saitoh, H.; Uemura, O., The Hydration Structure of NO3- in Concentrated Aqueous Sodium-Nitrate Solutions. Bulletin of the Chemical Society of Japan 1993, 66, 1919-1923.

209. Mason, P. E.; Neilson, G. W.; Dempsey, C. E.; Brady, J. W., Neutron Diffraction and Simulation Studies of $\mathrm{CsNO} 3$ and $\mathrm{Cs} 2 \mathrm{CO} 3$ Solutions. Journal of the American Chemical Society 2006, 128, 15136-15144.

210. Megyes, T.; Balint, S.; Peter, E.; Grosz, T.; Bako, I.; Krienke, H.; Bellissent-Funel, M. C., Solution Structure of Nano(3) in Water: Diffraction and Molecular Dynamics Simulation Study. Journal of Physical Chemistry B 2009, 113, 40544064.

211. Irish, D. E.; Davis, A. R., Interactions in Aqueous Alkali Metal Nitrate Solutions. Canadian Journal of Chemistry 1968, 46, 943-\&.

212. Riddell, J. D.; Irish, D. E.; Lockwood, D. J., Ion-Pair Formation in NaNO3-D2O Solutions - Raman and Infrared-Spectra, Partial Molal Volumes, Conductance, and Viscosity. Canadian Journal of Chemistry 1972, 50, 2951-\&.

213. Hester, R. E.; Plane, R. A., Vibrational Spectroscopic Study of Contact Ion Pairing between $\mathrm{Zn++}$ and NO3- in Water. The Journal of Chemical Physics 1966, 45, 4588-4593.

214. Frost, R. L.; James, D. W., Ion Ion Solvent Interactions in Solution .3. AqueousSolutions of Sodium-Nitrate. Journal of the Chemical Society-Faraday Transactions I 1982, 78, 3223-3234.

215. James, D. W.; Frost, R. L., Ion Ion Solvent Interactions in Solution - AqueousSolutions of Nitrates of Cations from Group-2a and Group-3a. Australian Journal of Chemistry 1982, 35, 1793-1806.

216. Chialvo, A. A.; Cummings, P. T., Microstructure of Ambient and Supercritical Water. A Direct Comparison between Simulation and Neutron Scattering Experiments. Journal of Physical Chemistry 1996, 100, 1309-1316.

217. Caminiti, R.; Cucca, P.; Dandrea, A., Hydration Phenomena in a Concentrated Aqueous-Solution of $\mathrm{Ce}(\mathrm{NO} 3) 3$ - X-Ray-Diffraction and Raman-Spectroscopy. Zeitschrift Fur Naturforschung Section a-a Journal of Physical Sciences 1983, 38, 533-539.

218. Caminiti, R.; Cucca, P.; Pintori, T., Hydration and Ion-Pairing in Concentrated Aqueous Mn(NO3)2 Solutions - an X-Ray and Raman-Spectroscopy Study. Chemical Physics 1984, 88, 155-161. 
219. Caminiti, R.; Radnai, T., X-Ray-Diffraction Study of a Concentrated Al(NO3)3 Solution. Zeitschrift Fur Naturforschung Section a-a Journal of Physical Sciences 1980, 35, 1368-1372.

220. Eigen, M.; Tamm, K., Schallabsorption in Elektrolytlosungen Als Folge Chemischer Relaxation .1. Relaxationstheorie Der Mehstufigen Dissoziation. Zeitschrift Fur Elektrochemie 1962, 66, 93-107.

221. Poirier, J. C.; DeLap, J. H., On the Theory of Ion Pairs in Solutions. Journal of Chemical Physics 1961, 35, 213-227.

222. Chialvo, A. A.; Cummings, P. T.; Cochran, H. D.; Simonson, J. M.; Mesmer, R. E., Na+_Cl- Ion Association in Supercritical Water. Journal of Chemical Physics 1995, 103, 9379-9387.

223. Chialvo, A. A.; Simonson, J. M., Solvation Behavior of Short-Chain Polystyrene Sulfonate in Aqueous Electrolyte Solutions: A Molecular Dynamics Study. Journal of Physical Chemistry B 2005, 109, 23031-23042.

224. Chialvo, A. A.; Ho, P. C.; Palmer, D. A.; Gruszkiewicz, M. S.; Cummings, P. T.; Simonson, J. M., $\mathrm{H}_{3} \mathrm{O}^{+} / \mathrm{Cl}^{-}$Association in High-Temperature Aqueous Solutions over a Wide Range of State Conditions. A Direct Comparison between Simulation and Electrical Conductance Experiment. Journal of Physical Chemistry B 2002, 106, 2041-2046.

225. Chialvo, A. A.; Simonson, J. M., Aqueous Na+Cl- Pair Association from LiquidLike to Steam-Like Densities Along near-Critical Isotherms. Journal of Chemical Physics 2003, 118, 7921-7929.

226. Chialvo, A. A.; Gruszkiewicz, M. S.; Cole, D. R., Ion-Pair Association in Ultrasupercritical Aqueous Environments: Successful Interplay among Conductance Experiments, Theory, and Molecular Simulations. Journal of Chemical \& Engineering Data 2010, 55, 1828-1836.

227. Kusalik, P. G.; Laaksonen, A.; Svishchev, I. M., Spatial Structure in Molecular Liquids. In Molecular Dynamics. From Classical to Quantum Methods, Balbuena, P. B.; Seminario, J. M., Eds. Elsevier: Amsterdam, 1999; pp 61-98.

228. Chialvo, A. A.; Vlcek, L., Ewald Summation Approach to Potential Models of Aqueous Electrolytes Involving Gaussian Charges and Induced Dipoles: Formal and Simulation Results. The Journal of Physical Chemistry B 2014, 118, 1365813670.

229. Vlcek, L.; Uhlik, F.; Moucka, F.; Nezbeda, I.; Chialvo, A. A., Thermodynamics of Small Alkali Metal Halide Cluster Ions: Comparison of Classical Molecular Simulations with Experiment and Quantum Chemistry. The Journal of Physical Chemistry A 2015, 119, 488-500.

230. Chialvo, A. A.; Moucka, F.; Vlcek, L.; Nezbeda, I., Vapor-Liquid Equilibrium and Polarization Behavior of the Gcp Water Model: Gaussian Charge-on-Spring Versus Dipole Self-Consistent Field Approaches to Induced Polarization. The Journal of Physical Chemistry B 2015, 119, 5010-5019. 


\section{FIGURE CAPTIONS}

Figure 1: Radial profile of $\Delta G_{M}^{\text {solv }}(r) \equiv G_{N i}(r)$ in our notation for a heavy water $\mathrm{NiCl}_{2}$ solution at ambient conditions taken from Ref. 65 with permission of the Royal Society

Figure 2: Radial profile of $\Delta G_{X}^{\text {solv }}(r) \equiv G_{C l}(r)$ in our notation for a heavy water $\mathrm{LiCl}$ solution at ambient conditions taken from Ref. 65 with permission of the Royal Society

Figure 3: Comparison between molecular-based simulation results ${ }^{58}$ and XRD experiment data ${ }^{92}$ for the concentration dependence of the $\mathrm{Ca}^{+2}-$ coordination, $\bar{n}_{C a}^{O}\left(r_{s}\right)$

Figure 4: Comparison between molecular-based simulation results ${ }^{58}$, XRD experiment data ${ }^{92}$, and EXAFS-XANES data from Fulton et al. ${ }^{83}$ for the concentration dependence of the $\mathrm{Cl}^{-} \cdots C \mathrm{Ca}^{+2}-$ coordination, $\bar{n}_{\mathrm{Ca}}^{\mathrm{Cl}}\left(r_{s}\right)$

Figure 5: Normalized first-order difference of neutron weighted distribution functions for the $6.4 \mathrm{mCaCl}_{2}$ in heavy- and null-water solution at ambient conditions under ${ }^{\text {nat }} \mathrm{Ca} /{ }^{44} \mathrm{Ca}$ substitution.

Figure 6: Normalized first-order difference of neutron weighted distribution functions for the $6.4 \mathrm{mCaCl}_{2}$ in heavy- and null-water solution at ambient conditions under ${ }^{n a t} \mathrm{Cl} /{ }^{37} \mathrm{Cl}$ substitution.

Figure 7: Normalized first-order difference of neutron weighted distribution functions for the $3.94 \mathrm{~m} \mathrm{NiCl}_{2}$ in heavy- and null-water solution at ambient conditions: under ${ }^{n a t} \mathrm{Ni} /{ }^{62} \mathrm{Ni}$ substitution. 
Figure 8: Normalized first-order difference of neutron weighted distribution functions for the $3.94 \mathrm{~m} \mathrm{NiCl}_{2}$ in heavy- and null-water solution at ambient conditions under ${ }^{n a t} \mathrm{Cl} /{ }^{37} \mathrm{Cl}$ substitution.

Figure 9: Normalized first-order difference of neutron weighted distribution functions for the $3.94 \mathrm{~m} \mathrm{NiCl}_{2}$ heavy-water solution at ambient conditions under "nullnickel" environment and ${ }^{n a t} \mathrm{Cl} /{ }^{37} \mathrm{Cl}$ substitution.

Figure 10: Normalized first-order difference of neutron weighted distribution functions for the $14 \mathrm{~m} \mathrm{LiCl}$ in heavy- and null-water solutions at ambient conditions under ${ }^{n a t} \mathrm{Cl} /{ }^{37} \mathrm{Cl}$ substitution.

Figure 11: Concentration dependence of the coordination numbers of aqueous $\mathrm{LiCl}$ solutions according to conventional integration of the neutron-weighted distribution functions, Eqn. (9), their CIP corrected (“corr”) counterparts, and the corresponding degree of CIP association.

Figure 12: Concentration dependence of the contact $L i \cdots C l$ coordination $\bar{n}_{C l}^{L i}(C I P)$ and the corresponding degree of pair association $\alpha_{L i C l}(C I P)$ in aqueous $\mathrm{LiCl}$ solutions from molecular simulation and a variety of experimental approaches.

Figure 13: Normalized first-order difference of neutron weighted distribution functions for the $14 \mathrm{~m} \mathrm{LiCl}$ heavy-water solution at ambient conditions under "nulllithium" environment and ${ }^{n a t} \mathrm{Cl} /{ }^{37} \mathrm{Cl}$ substitution.

Figure 14: Normalized first-order difference of neutron weighted distribution functions for the $6.16 \mathrm{miNO}_{3}$ in heavy- and null-water solution at ambient conditions under ${ }^{14} N /{ }^{15} N$ substitution. 
Figure 15: Normalized first-order difference of neutron weighted distribution functions for the $6.16 \mathrm{miNO}_{3}$ in heavy- and null-water solution at ambient conditions under ${ }^{\text {nat }} \mathrm{O} /{ }^{18} \mathrm{O}$ substitution.

Figure 16: Radial pair distribution function $g_{-+}(r)$, as well as their corresponding ionpair radial distribution functions $G_{-+}(r)$ and degree of ion-pair association $\alpha_{-+}(r)$ for the $6.4 \mathrm{~m} \mathrm{CaCl}_{2}$ aqueous solution at ambient conditions.

Figure 17: Radial pair distribution function $g_{-+}(r)$, as well as their corresponding ionpair radial distribution functions $G_{-+}(r)$ and degree of ion-pair association $\alpha_{-+}(r)$ for the $3.94 \mathrm{~m} \mathrm{NiCl}_{2}$ aqueous solution at ambient conditions.

Figure 18: Radial pair distribution function $g_{-+}(r)$, as well as their corresponding ionpair radial distribution functions $G_{-+}(r)$ and degree of ion-pair association $\alpha_{-+}(r)$ for the $14 m \mathrm{LiCl}$ aqueous solution at ambient conditions.

Figure 19: Radial pair distribution function $g_{-+}(r)$, as well as their corresponding ionpair radial distribution functions $G_{-+}(r)$ and degree of ion-pair association $\alpha_{-+}(r)$ for the $6.16 m \mathrm{LiNO}_{3}$ aqueous solution at ambient conditions.

Figure 20: Ionic strength dependence of the estimated degree of ion-pair association $\alpha_{\mathrm{CaCl}^{+}}(C I P)$ from the corresponding experimentally measured 'concentration products' in comparison with microstructure-based values from molecular simulation of model $\mathrm{CaCl}_{2}$ aqueous solutions.

Figure 21: Spatial pair distribution functions $g_{L i N}(r, \theta)_{\phi=c o n s t}$ for the $6.16 m \mathrm{LiNO}_{3}$ aqueous solution at ambient conditions: top at $\phi=0^{\circ}$ and bottom at $\phi=60^{\circ}$ according to the set of axes described on the right. 
Figure 1

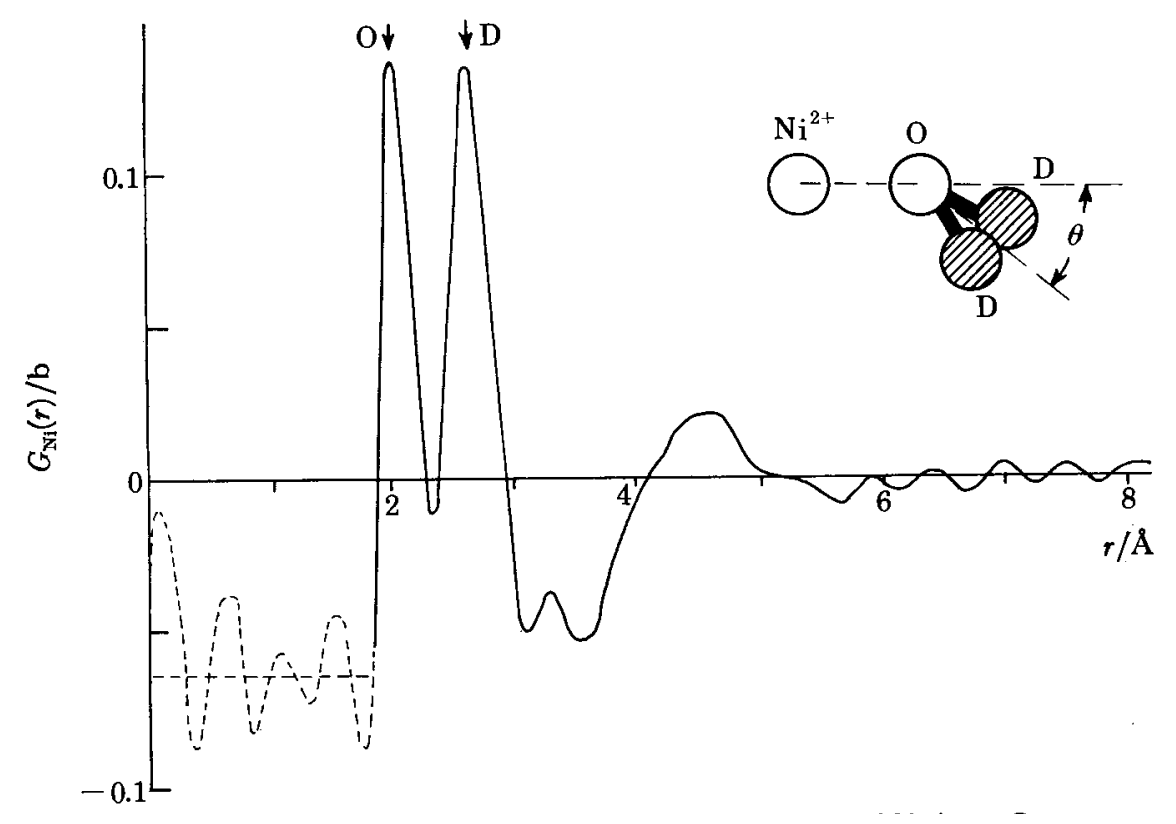


Figure 2

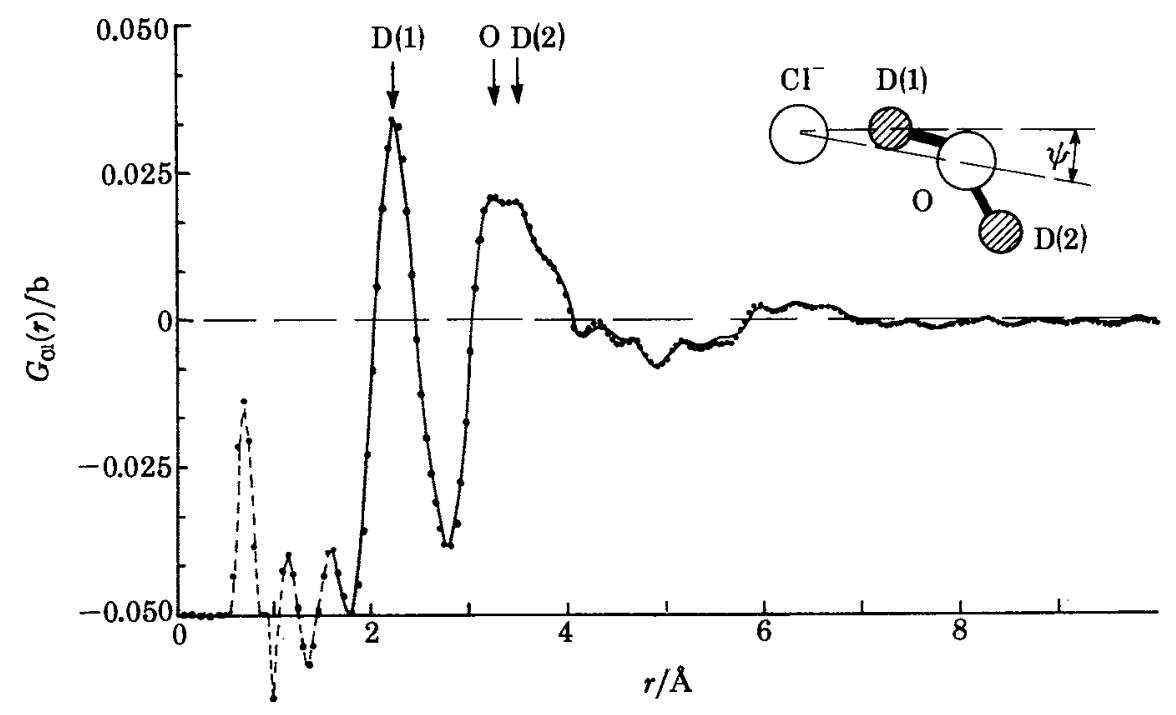


Figure 3

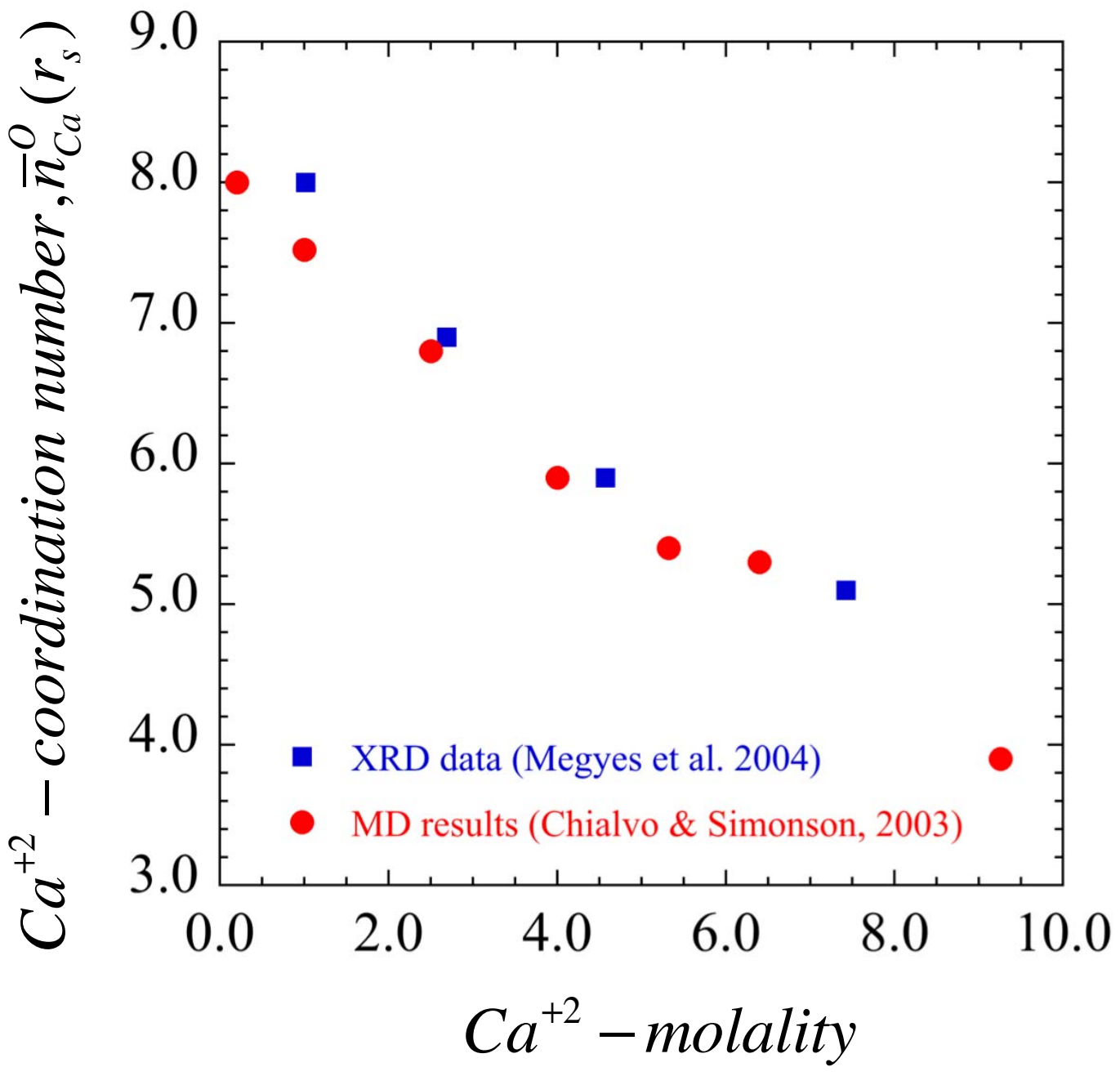


Figure 4

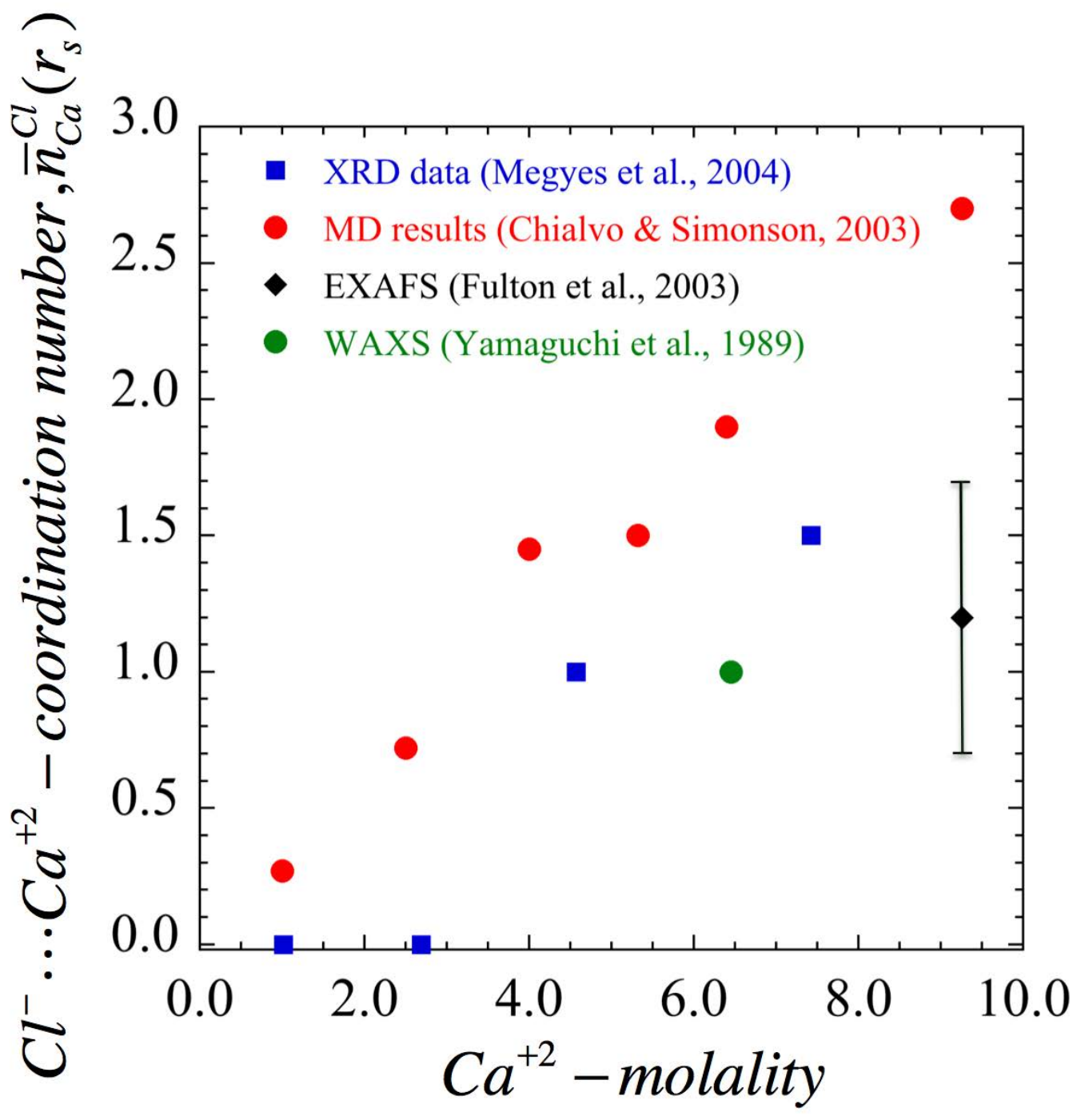


Figure 5

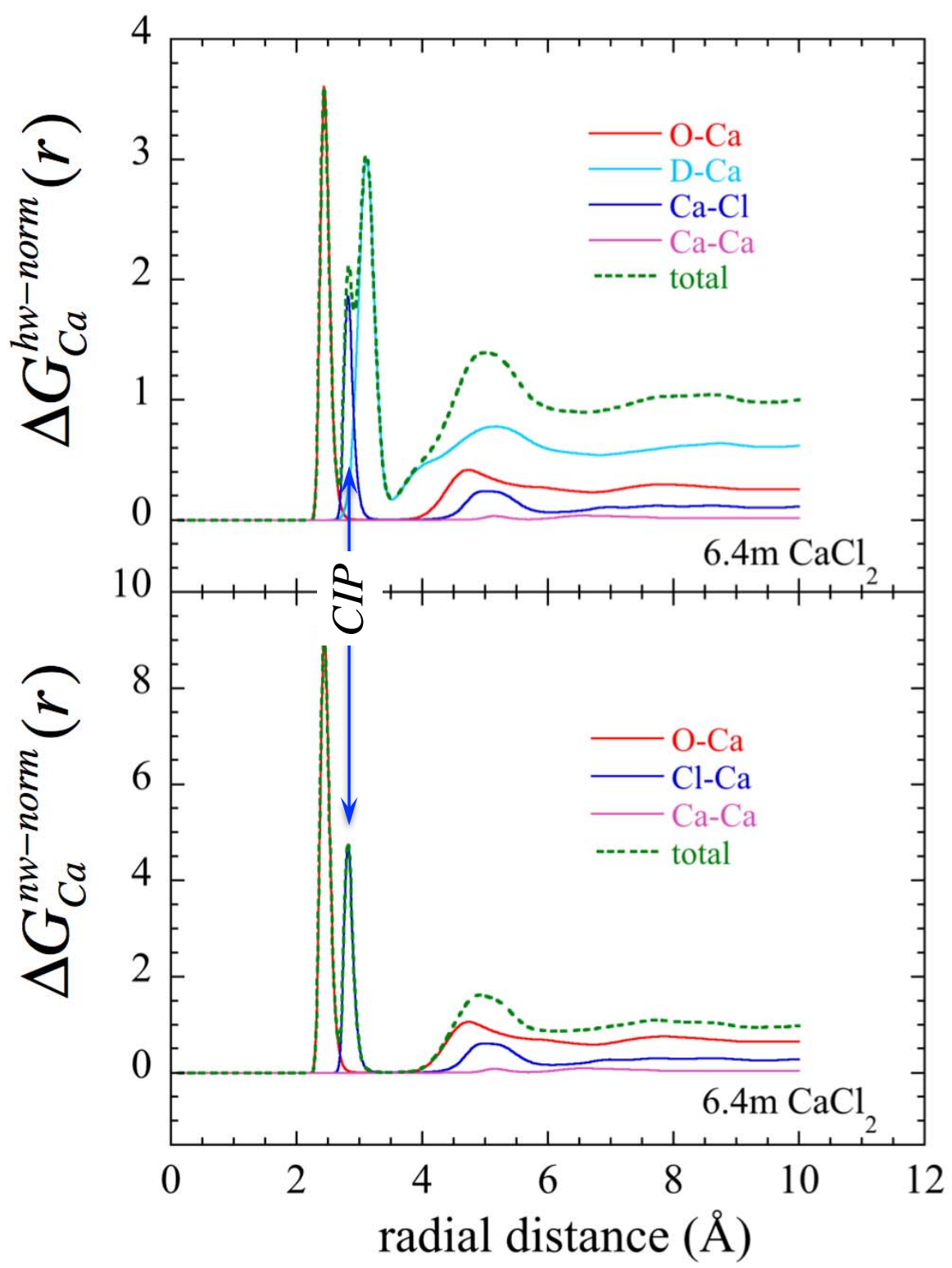


Figure 6

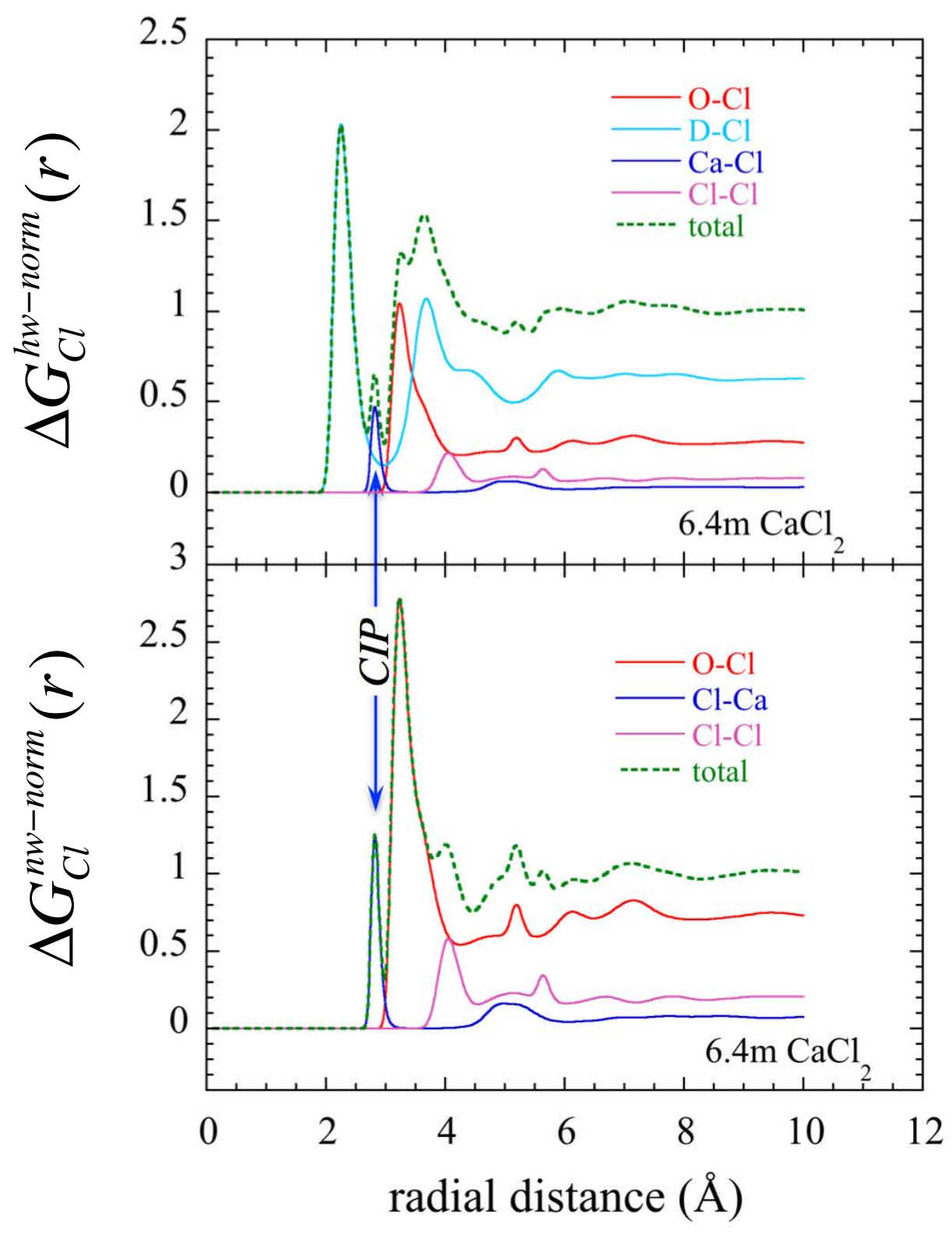


Figure 7

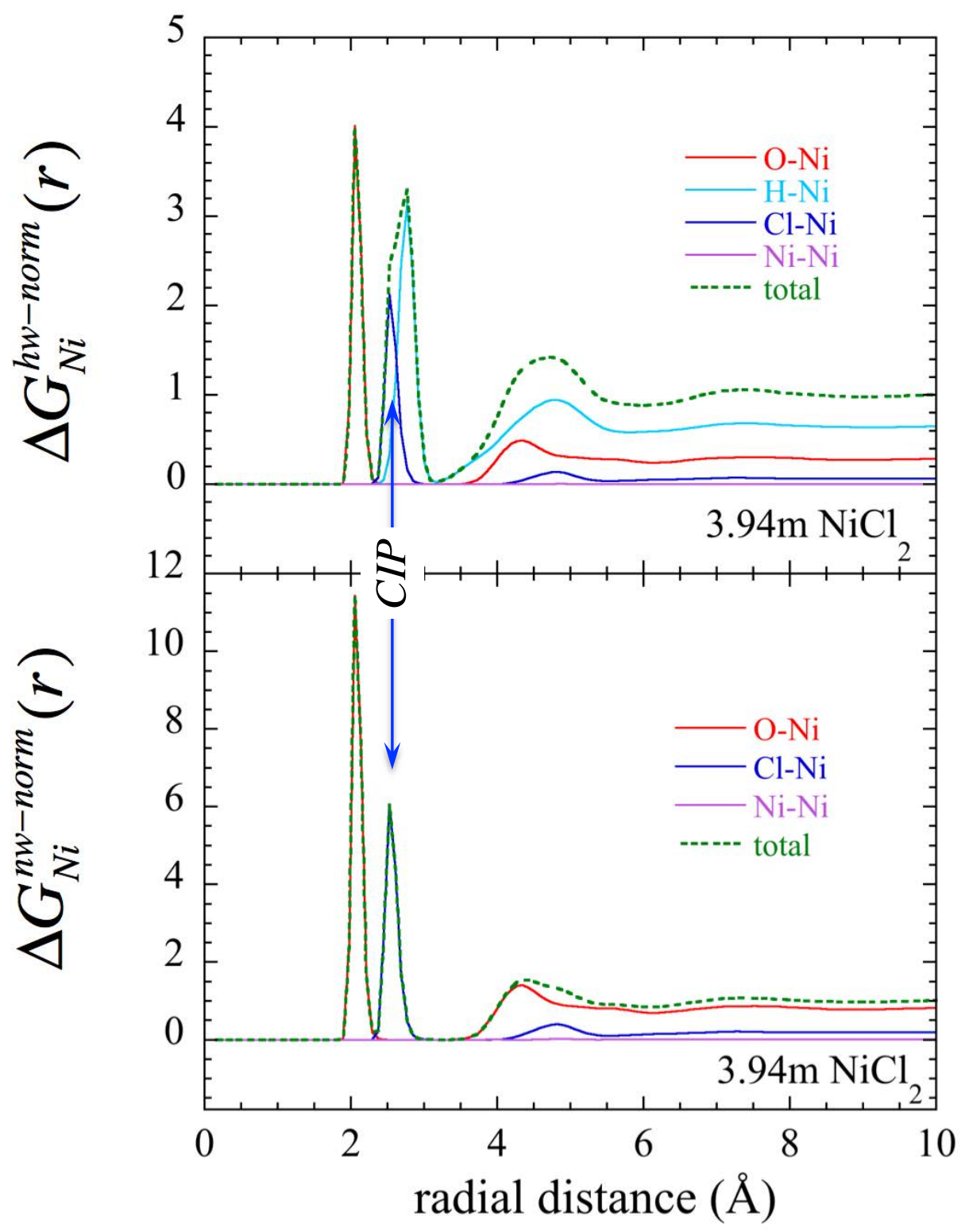


Figure 8

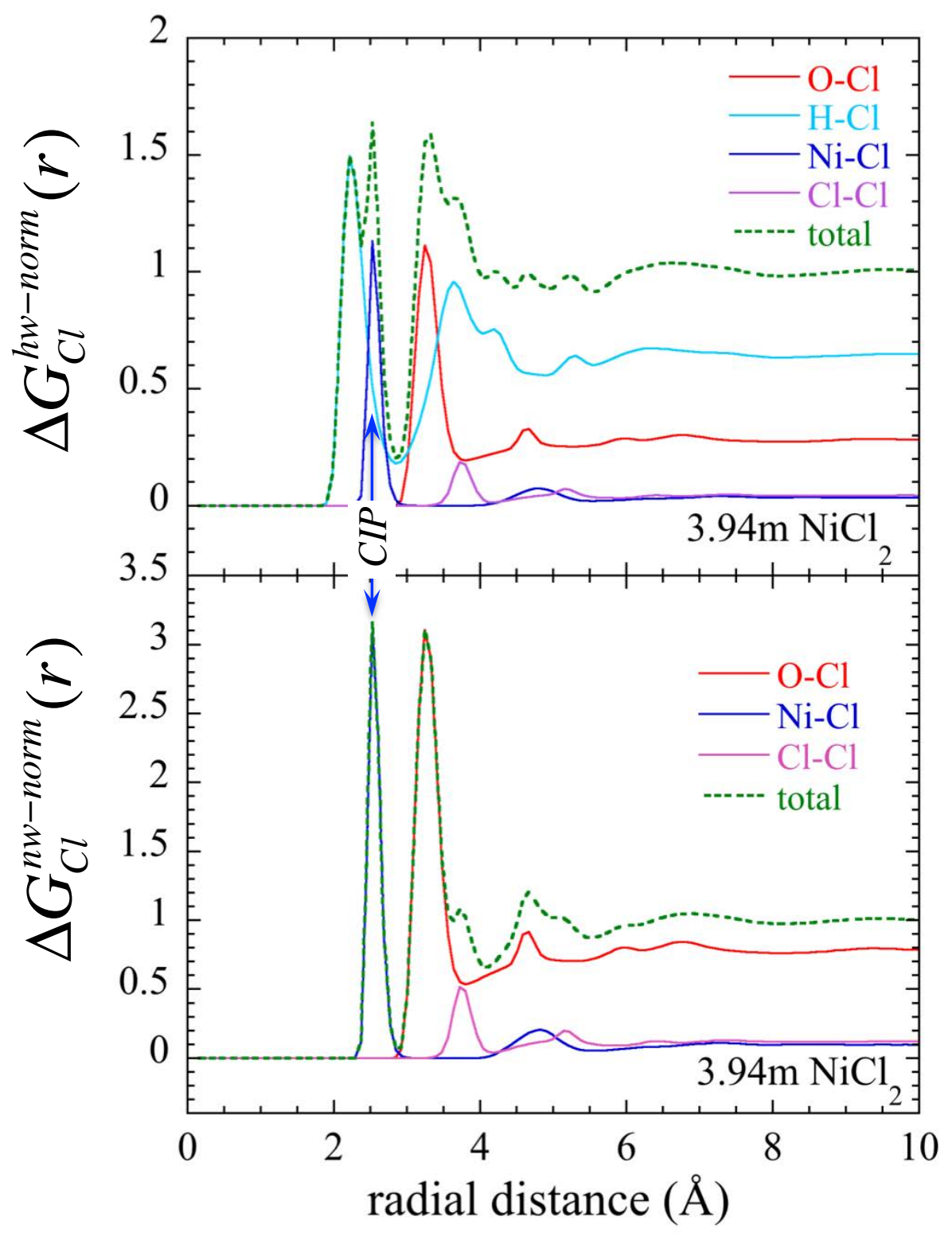


Figure 9

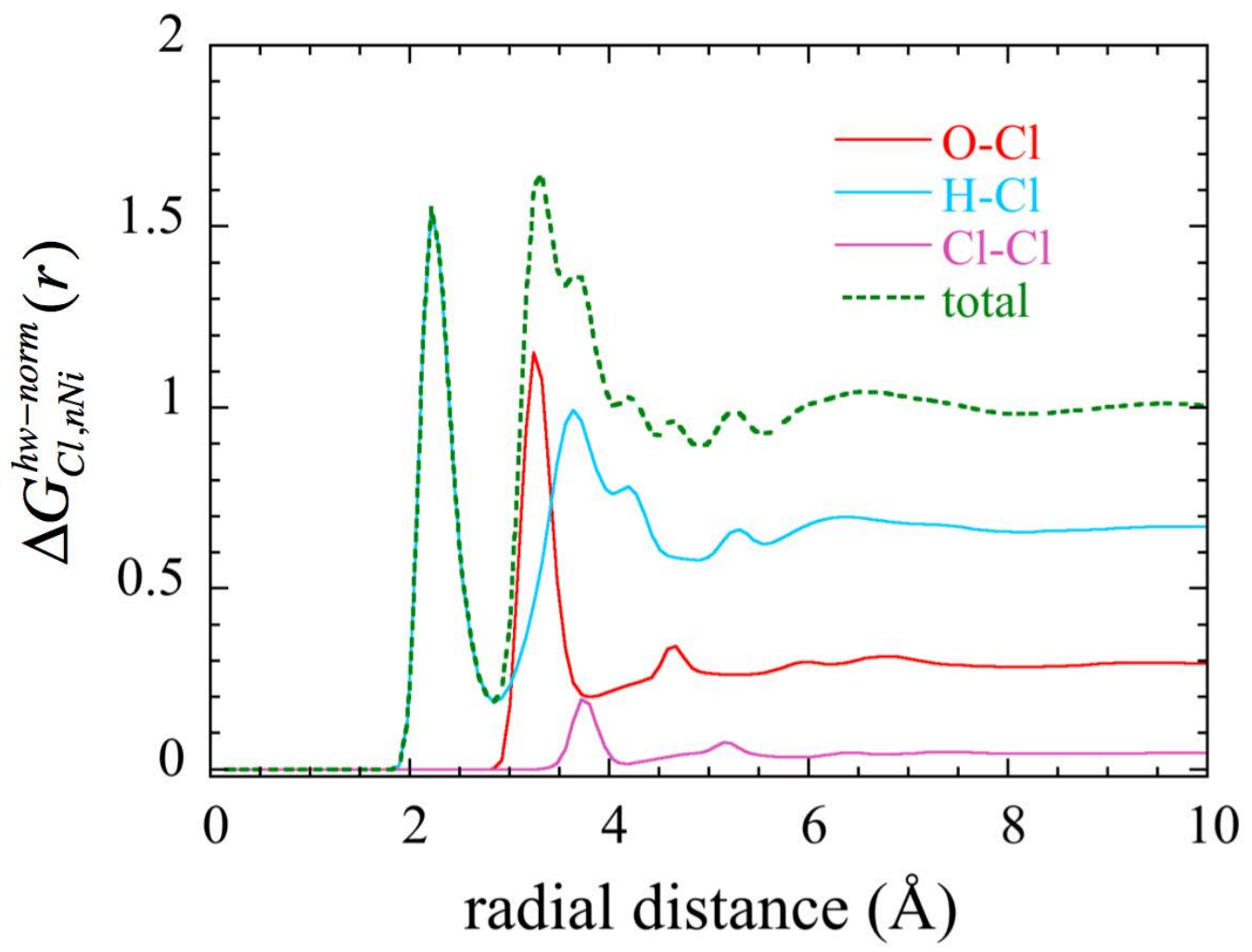


Figure 10

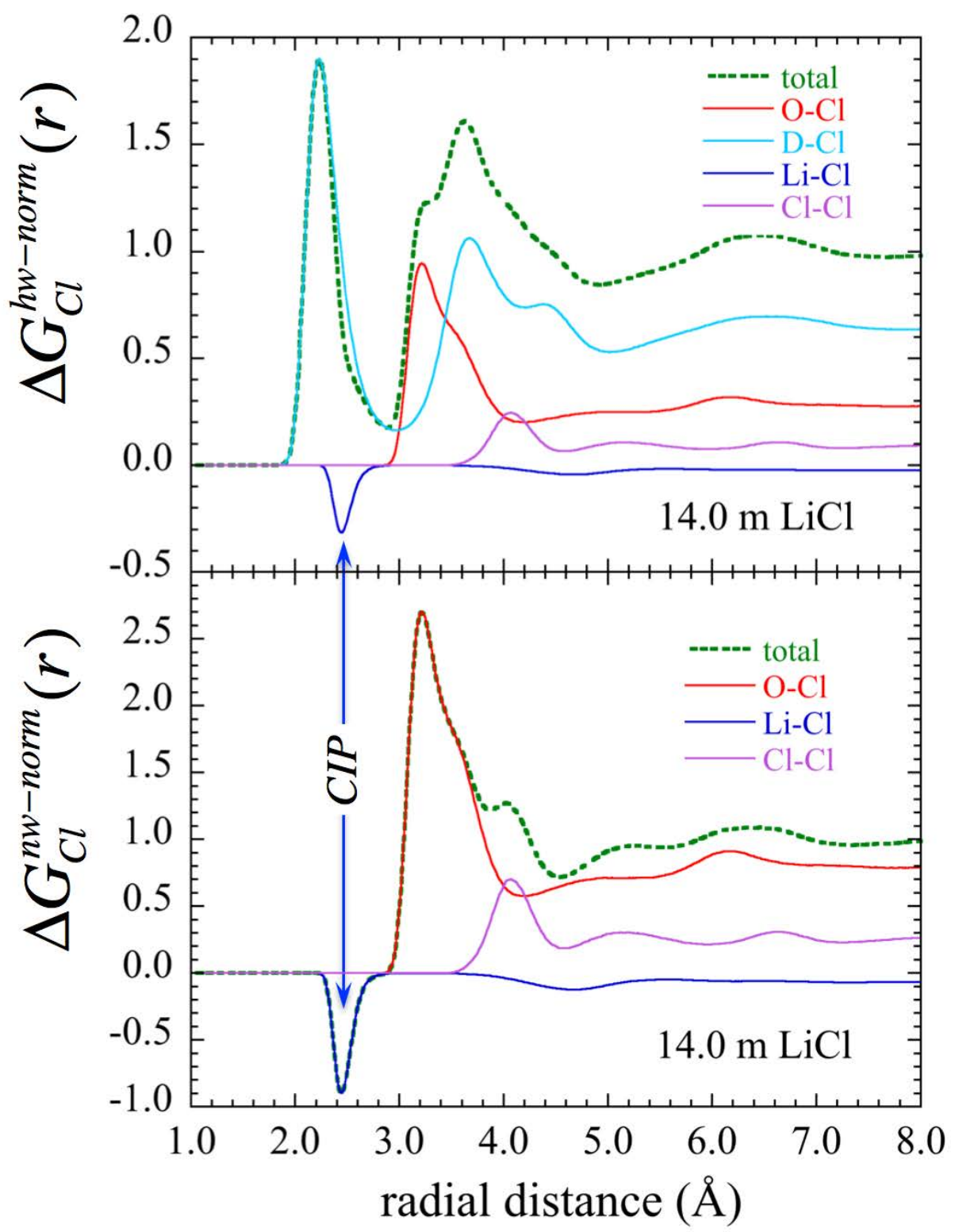


Figure 11

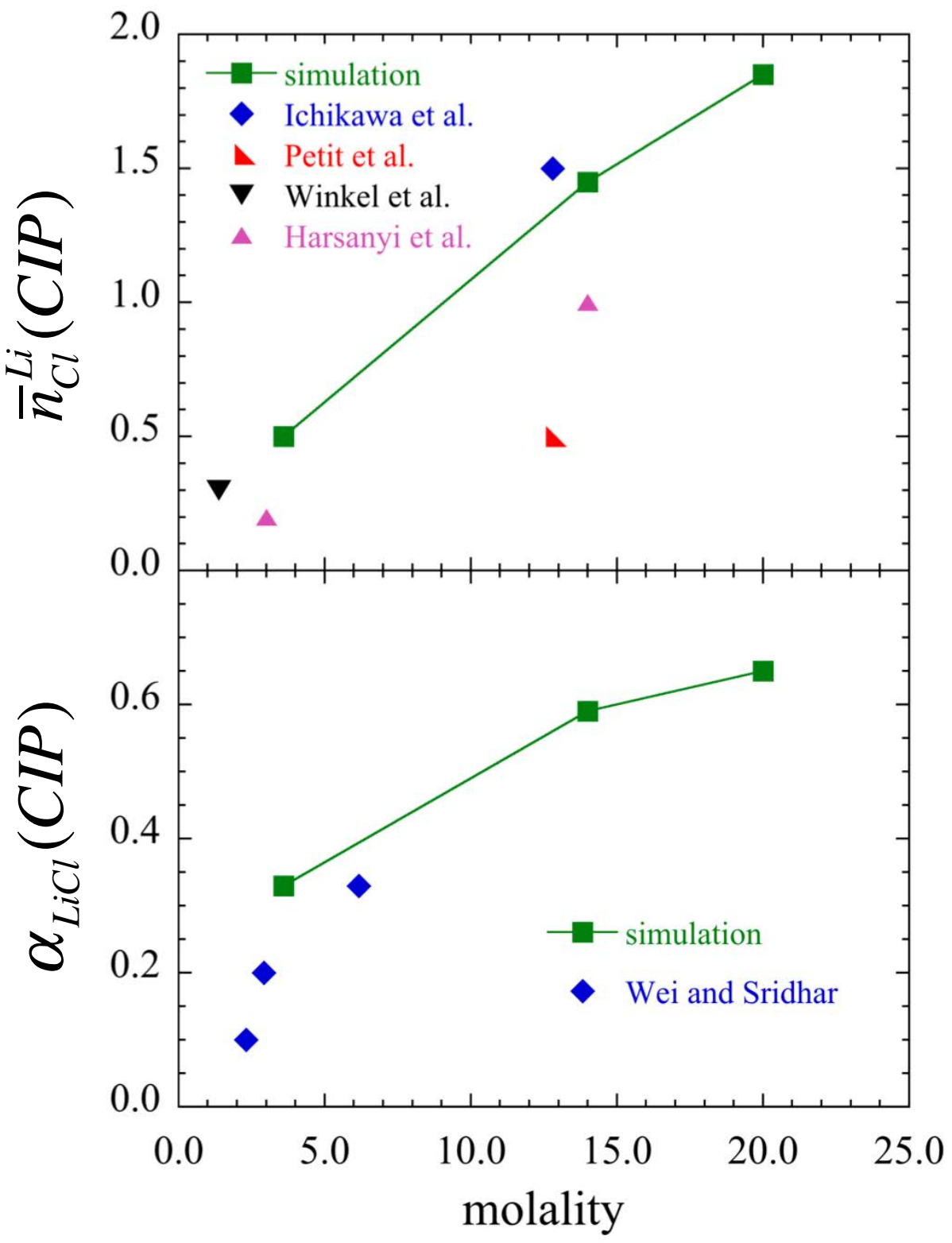


Figure 12

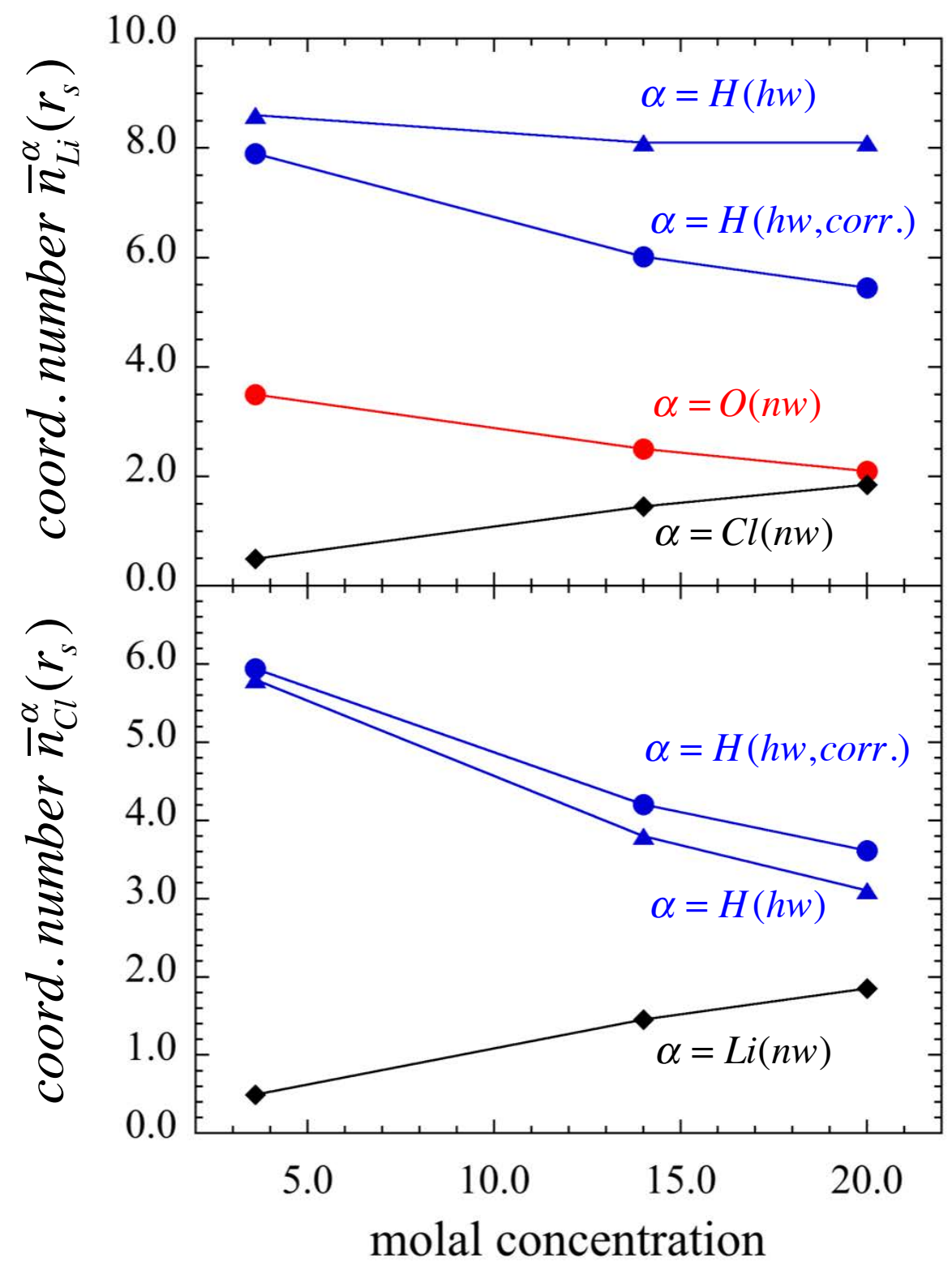


Figure 13

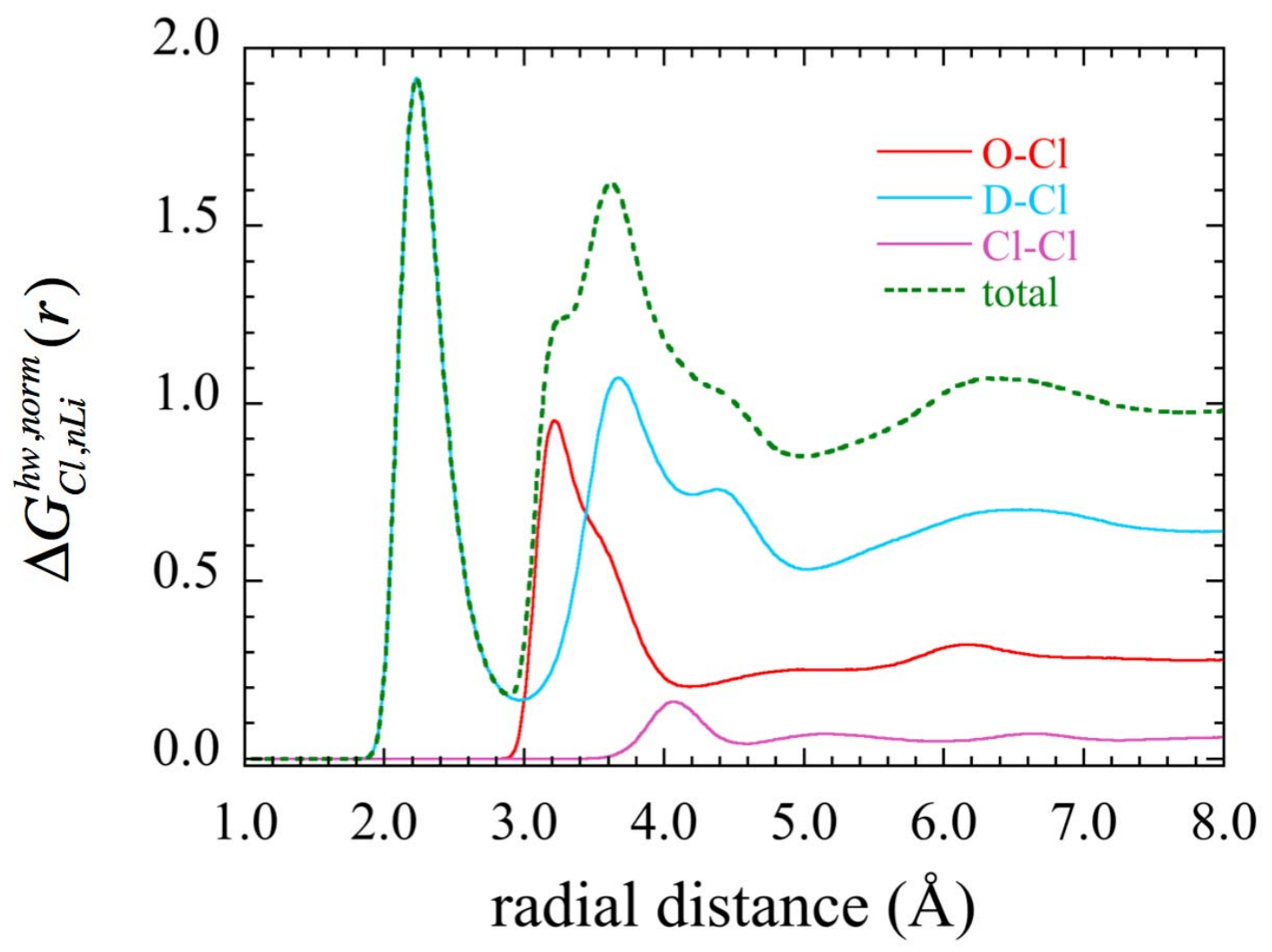


Figure 14

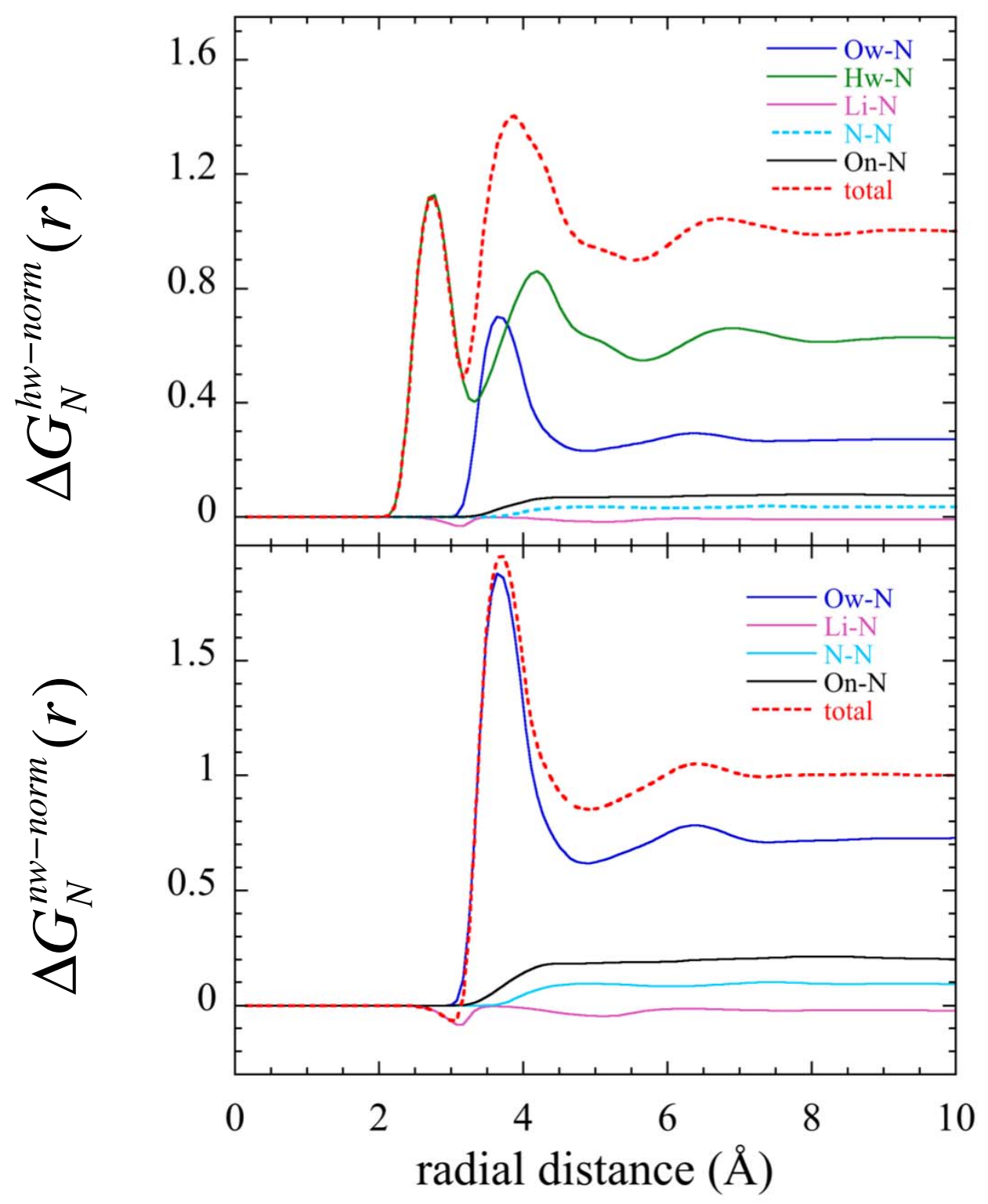


Figure 15

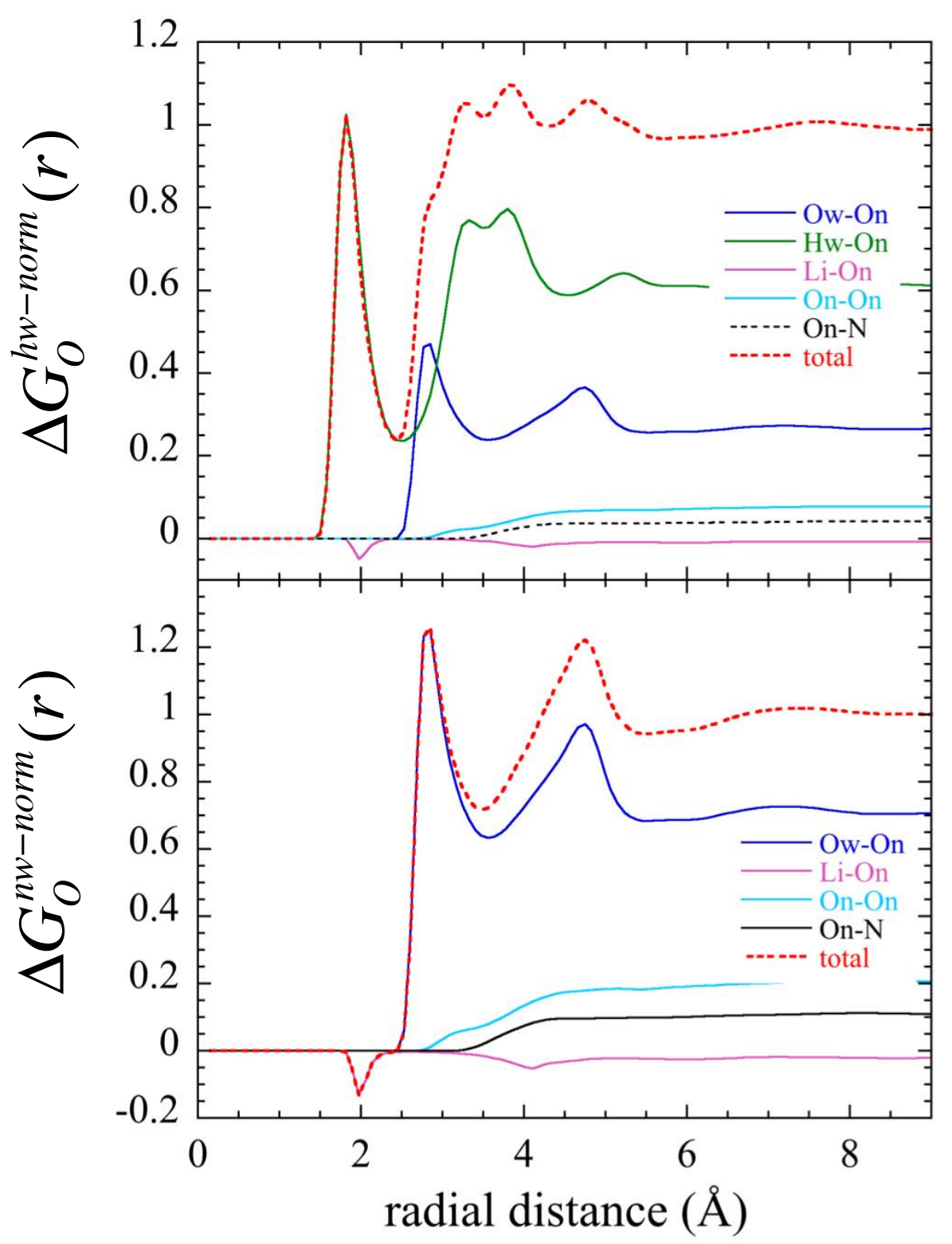


Figure 16

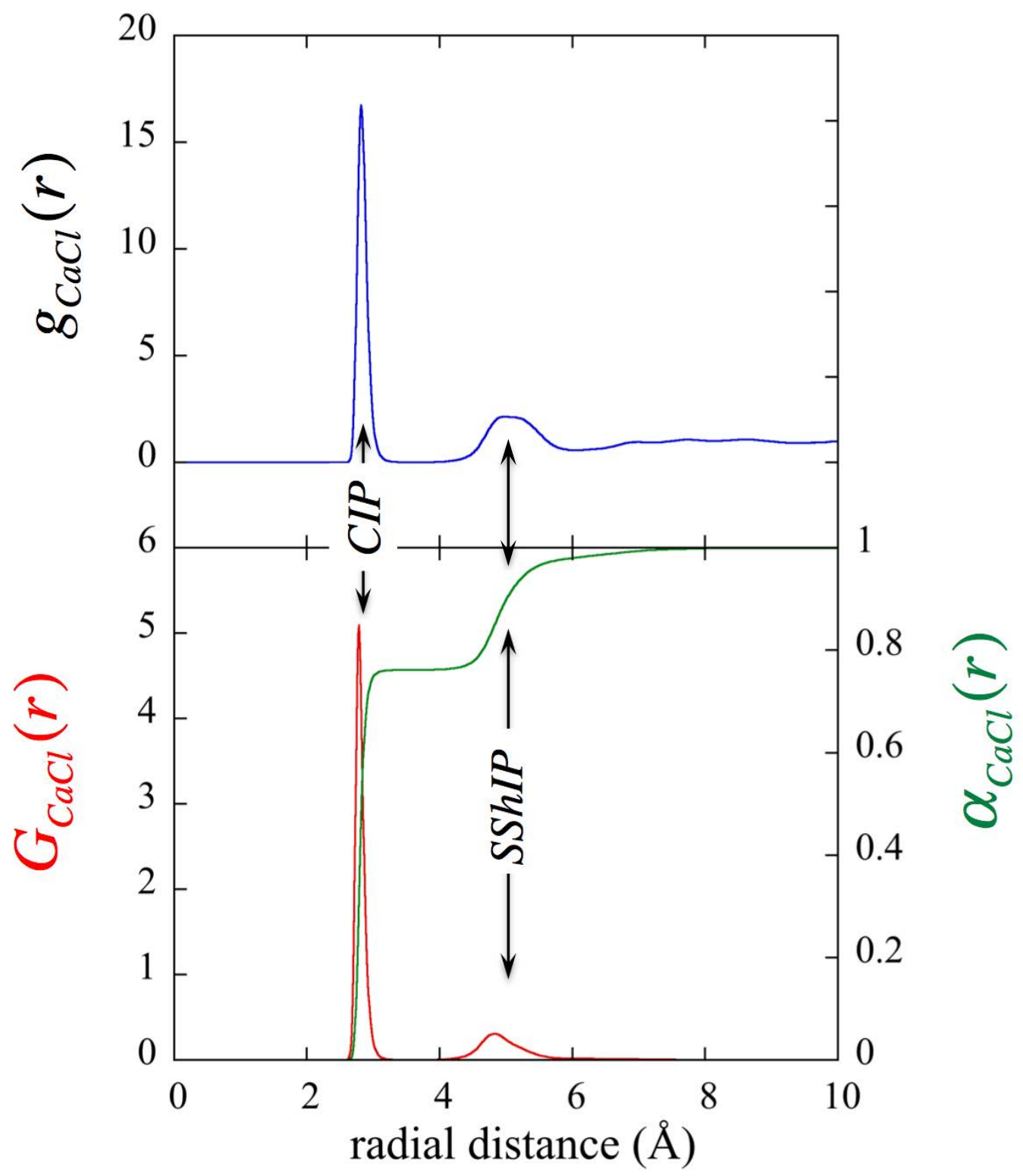


Figure 17

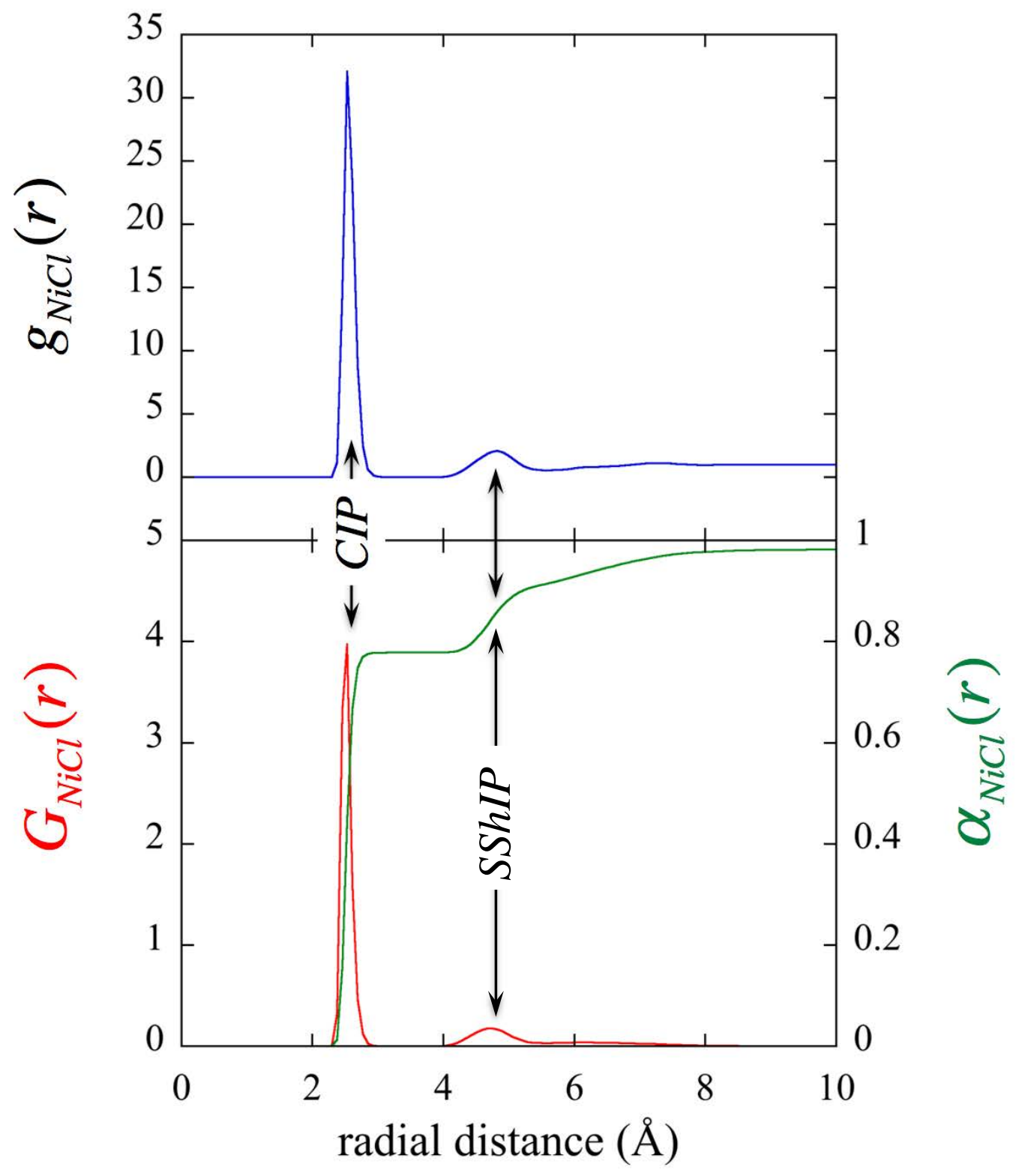


Figure 18

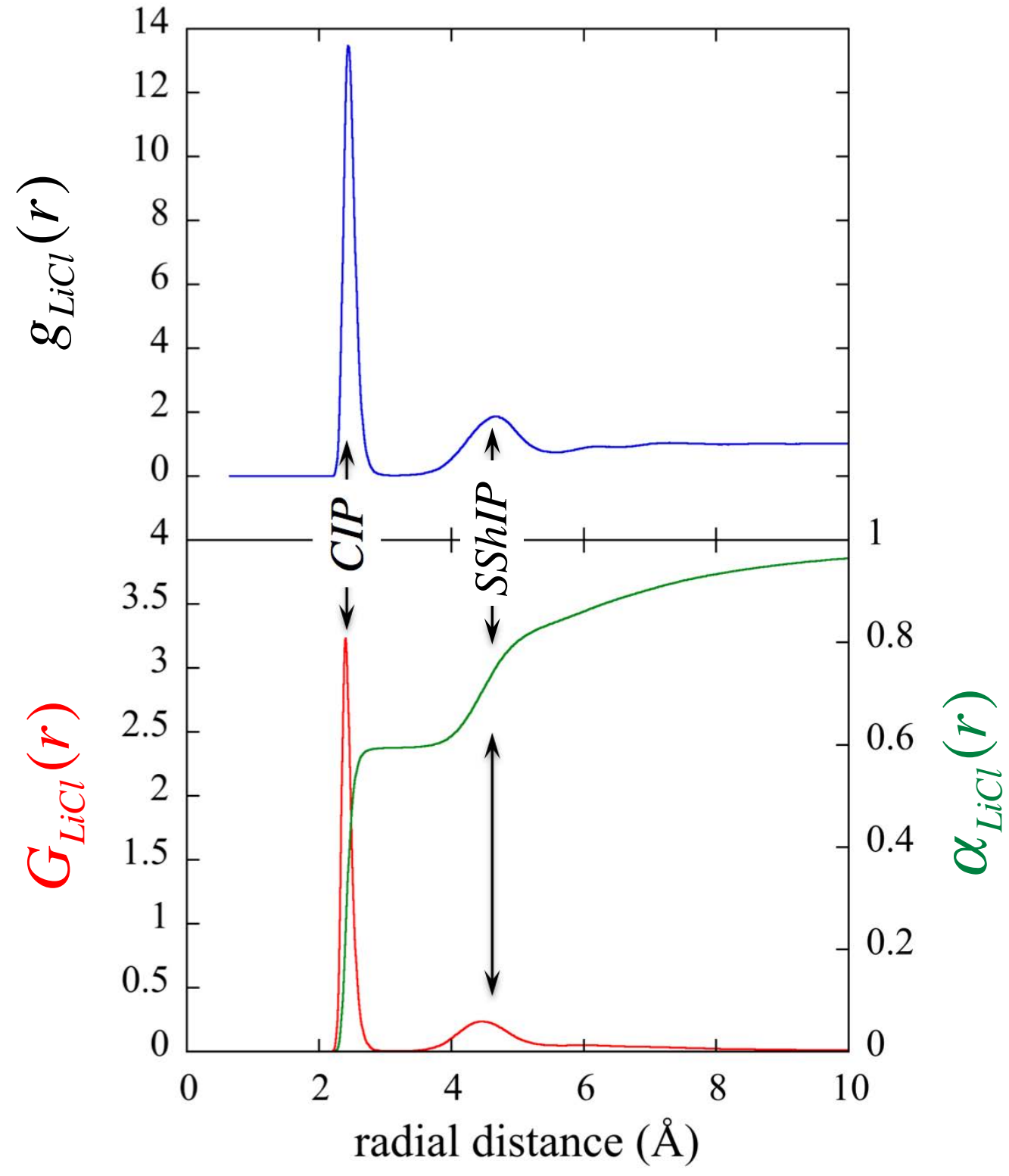


Figure 19

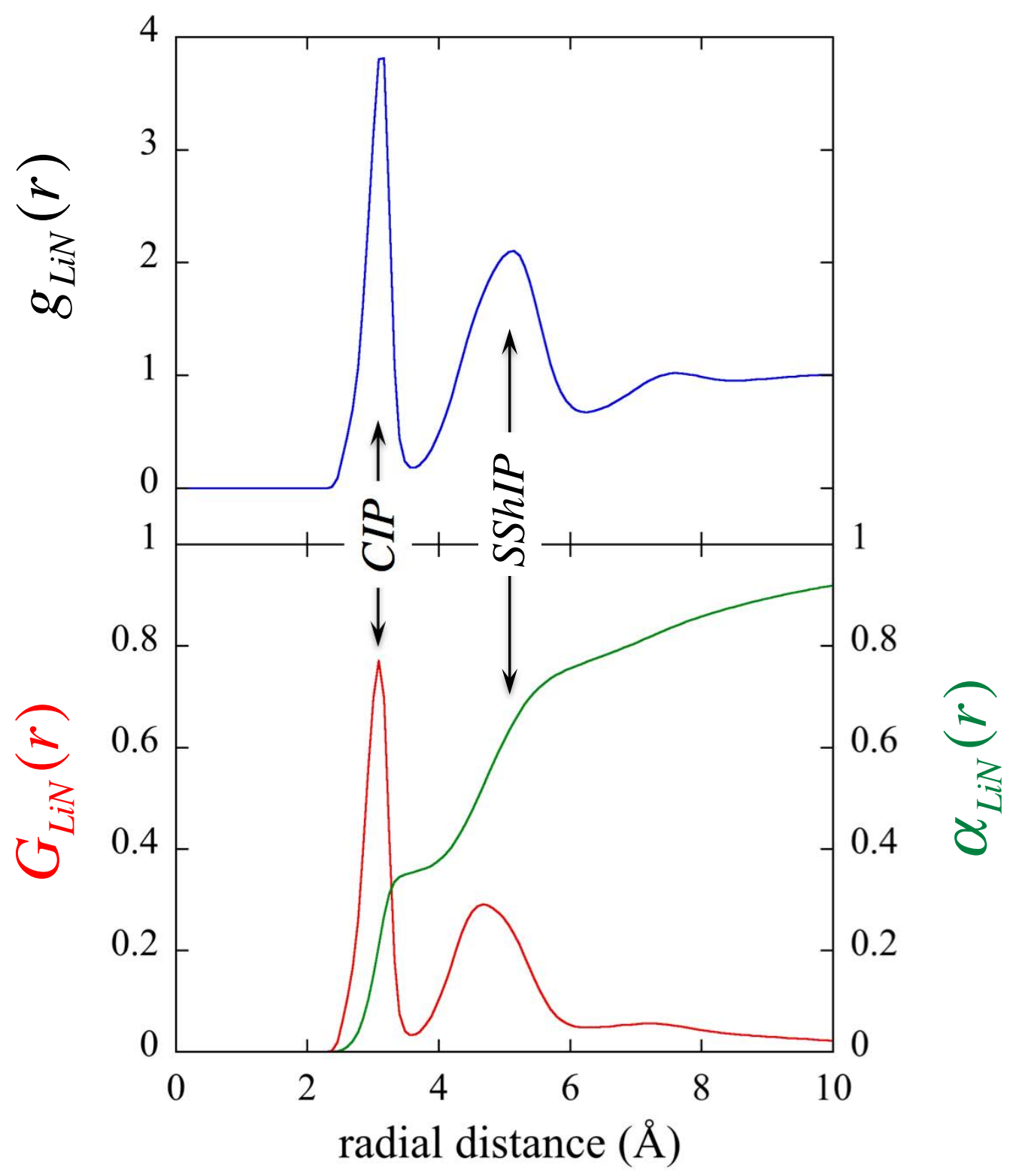


Figure 20

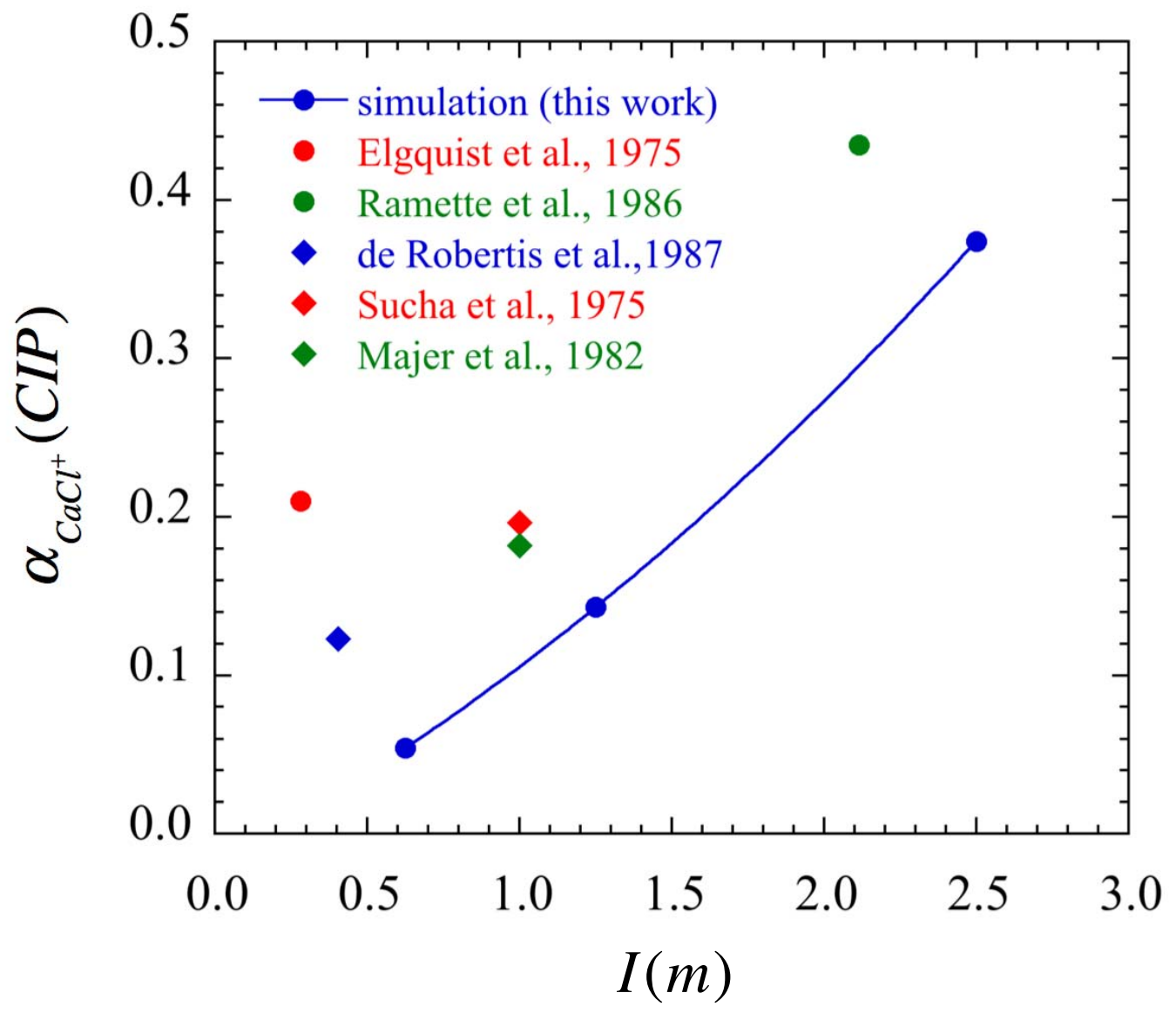


Figure 21
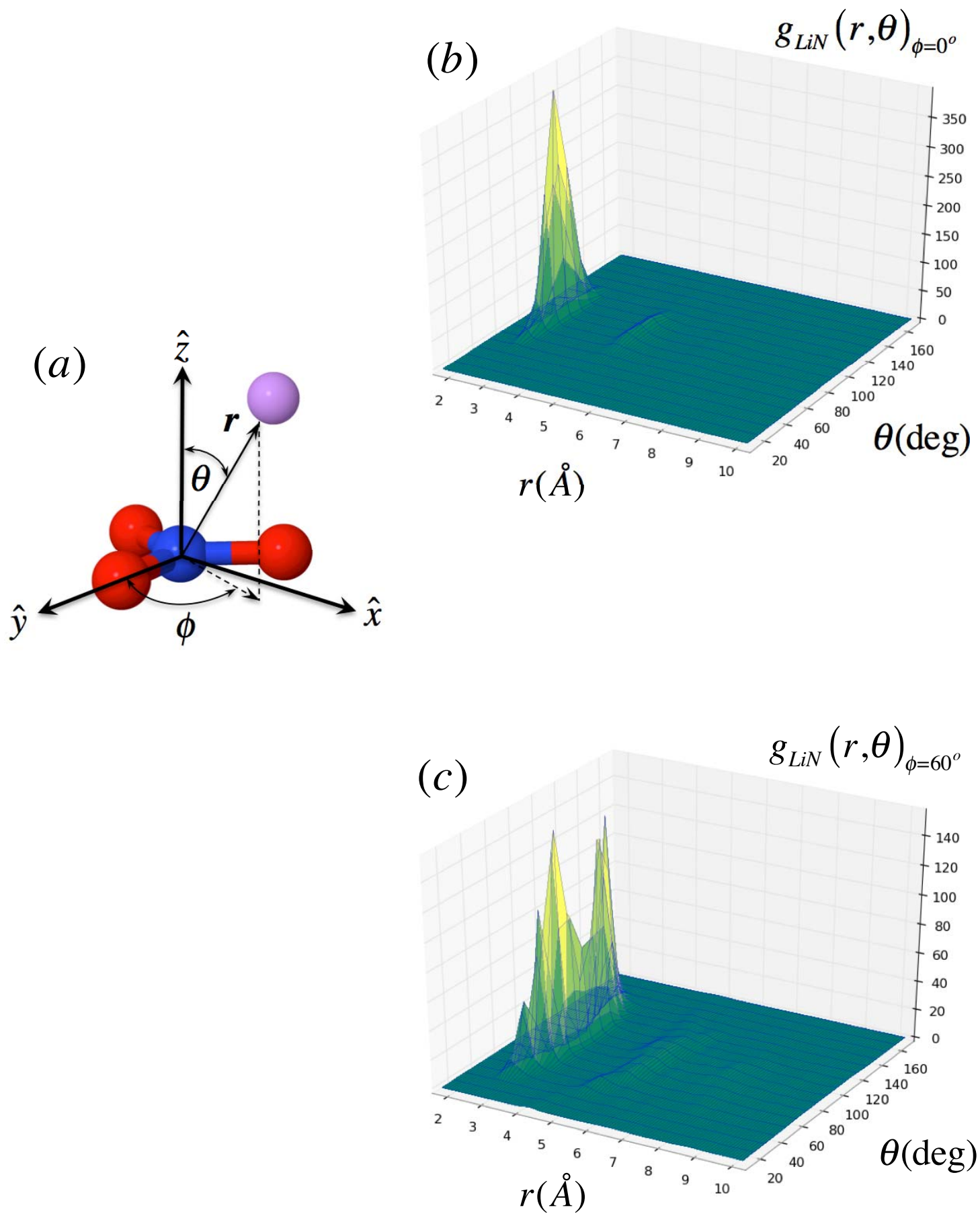